\title{
Collapse of Transient Gels in Colloid-Polymer Mixtures
}

Laura Starrs

Thesis submitted for the degree of Doctor of Philosophy

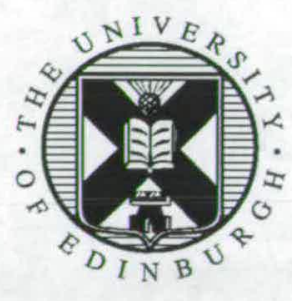

Department of Physics and Astronomy

University of Edinburgh

1999 


\section{Abstract}

The addition of non-adsorbing polymer to a suspension of colloidal hard-spheres causes phase separation via the depletion mechanism. At high enough concentration of polymer a variety of non-equilibrium aggregation behaviour is observed. Transient gelation is one such behaviour observed at the highest polymer concentrations.

Transient colloid-polymer gels are metastable space-filling particle networks. They persist for some finite time before suddenly collapsing to form a dense sediment. Thus transient gels exhibit "delayed sedimentation" which is a phenomenon observed in many weakly aggregated suspensions.

We have studied the collapse process occurring in the bulk of a gelled suspension using dark-field imaging and ultrasonic concentration profiling. At low polymer concentrations we observe delayed sedimentation behaviour. At the highest polymer concentration we observe a change in the settling behaviour. The suspension continuously sediments at a rate comparable to the initial slow settling rate of gels exhibiting delayed sedimentation. This is known as "creeping sedimentation".

We have also investigated the effect of varying suspension height and width on the delay time of a gel. We have found a critical height below which the gel exhibits a size-dependent delay time. Above this critical height the delay time is independent of height. The same behaviour is found when width is varied. We find good agreement between the results of this experimental study and a recent theory. 


\section{Acknowledgements}

Special thanks go to my supervisors Wilson Poon, Peter Pusey and Margaret Robins who between them have guided me through the jungle that is soft matter physics.

On the technical front I would like to thank Andy Schofield for making large quantities of PMMA colloid without which none of this work would have been possible. I would also like to thank "Uncle" Steve Illet for showing me the ropes all those years ago and teaching me how to live life in comfort. I'm also indebted to Falk Renth and Jerome Arrault for sharing with me their vast knowledge of all things optical (and doughnuts). Thanks also to David Hibberd at the Institute of Food Research for help and advice in performing the ultrasonics experiments and directing me to the best Thai restaurant in Norwich.

For useful discussion and reading various parts of the thesis manuscript I'd like to thank Mark Haw and Mike Evans. I'd also like to acknowledge the rest of the "gelsters" with whom I have had interesting scientific discourse: Mike Cates, Steve Meeker, and Abdellatif Mousaïd.

I think that about wraps it up except to say a big thankyou to all the softies and the members of E=M.C.C. for keeping me sane(ish). Finaly, to my family for all their support during the last 25 years and to Mark for just being there. 


\section{Contents}

Abstract $\quad$ iii

Declaration

Acknowledgements vii

1 Introduction 1

1.1 What is a Colloid $? \ldots \ldots \ldots \ldots \ldots \ldots$

1.2 Why Study Colloids $? \ldots \ldots \ldots \ldots \ldots$

1.3 Hard-Sphere Colloids . . . . . . . . . . . . . . . . . 2

1.3.1 Building a Model Hard Sphere System . . . . . . . . . . . . . 4

1.3 .2 Our Model System . . . . . . . . . . . . . 7

1.4 Thesis Summary $\ldots \ldots \ldots \ldots \ldots \ldots \ldots \ldots$

2 Colloid-Polymer Mixtures $\quad 9$

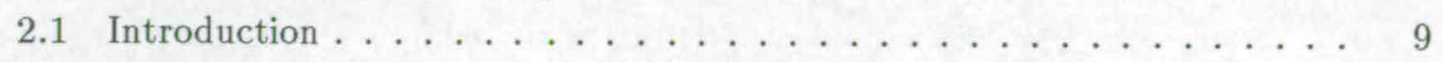

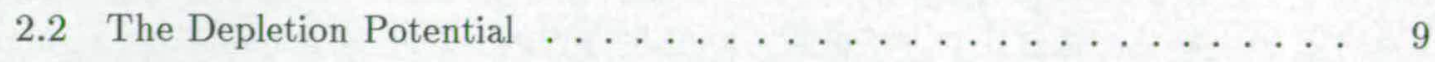

2.3 Predicting the Phase Behaviour . . . . . . . . . . . 12 
2.4 Observed Equilibrium Phase Behaviour . . . . . . . . . . . . . 14

2.5 Non-equilibrium Behaviour . . . . . . . . . . . . 16

2.5.1 Onset of Non-equilibrium Behaviour . . . . . . . . . . 17

2.5.2 Influence of Gravity on the Gel Boundary . . . . . . . . . 19

2.5.3 The Sharpness of the Non-equilibrium Boundary . . . . . . . . 21

2.6 Transient Gel Collapse . . . . . . . . . . . . . . . . . . 22

2.6.1 Delayed Sedimentation . . . . . . . . . . . . . 22

2.6.2 Predicting the Delay Time . . . . . . . . . . . . . 24

2.6.3 Imaging of Gel Collapse . . . . . . . . . . . . . 26

2.6.4 Low Shear Rheology of Transient Gels . . . . . . . . . . . . 27

3 Experimental Methods $\quad 29$

3.1 Introduction . . . . . . . . . . . . . . . 29

3.2 Dark-Field Imaging . . . . . . . . . . . . . . . . . 29

3.2 .1 Theoretical Background ................ 30

3.2 .2 Experimental Setup . . . . . . . . . . . . . 33

3.2 .3 Image Interpretation . . . . . . . . . . . 35

3.3 Ultrasonic Time-of-Flight Measurements . . . . . . . . . . . . . 36

3.3.1 Measuring Time-of-Flight . . . . . . . . . . . . . . 37

3.3.2 Calculating the Speed of Sound in the Sample . . . . . . . 40

3.3.3 Calculating the Sample Concentration . . . . . . . . . . . . . 41

3.3 .4 Multiple Scattering . . . . . . . . . . . . . . . . 43 


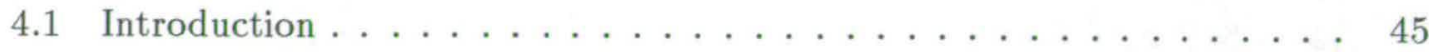

4.2 The Model System . . . . . . . . . . . . . . . . . . . 46

4.3 Sample Preparation . . . . . . . . . . . . . . . . . . 47

4.3 .1 The Sample Cell . . . . . . . . . . . . . . . . . . . . . . 48

4.3 .2 Errors ........................... 49

4.4 The Sedimentation Profile . . . . . . . . . . . . . 51

4.4.1 Measuring the Sedimentation Profile . . . . . . . . . . 52

4.4.2 Extracting the Delay Time and Settling Velocities . . . . . . 53

4.4 Errors ......................... 54

4.5 Delayed Sedimentation Observations . . . . . . . . . . . . . . 54

4.5.1 Short and Intermediate Delay Times . . . . . . . . . . 56

4.5 .2 Long Delay Times . . . . . . . . . . . . . . . . 60

4.6 Creeping Sedimentation Observations . . . . . . . . . . . . 61

4.7 Discussion ............................ 63

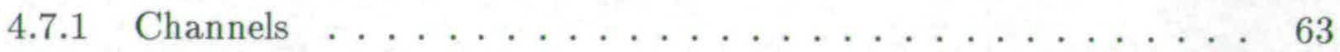

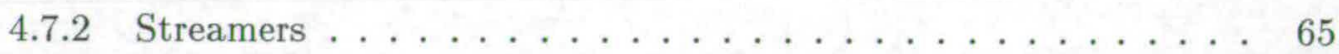

4.7 .3 Bright Bands . . . . . . . . . . . . . 66

4.7 .4 Cracks .............................. 68

4.7 .5 Creeping Sedimentation ................ 68 
5.1 Introduction . . . . . . . . . . . . . . . . . 69

5.2 The Model System . . . . . . . . . . . . . . . . . 69

5.2 .1 Sample Preparation ................ 70

5.3 Experimental Method . . . . . . . . . . . . . . 70

5.4 Results........................... 71

5.4.1 Short and Intermediate Delay Times . . . . . . . . . . . . 72

5.4 Long Delay Times . . . . . . . . . . . . . . . . . . 74

5.4 .3 Creeping Sedimentation ............... 76

5.5 Interpretation of Concentration Profiles $\ldots \ldots \ldots 77$

5.5 .1 Delayed Sedimentation . . . . . . . . . . . . 78

5.5 .2 Creeping Sedimentation ................ 81

6 Size Effects

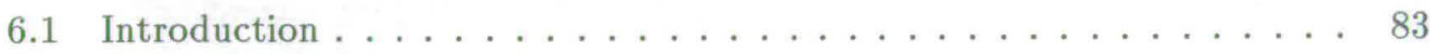

6.2 Strong and Weak Gels . . . . . . . . . . . . . . . 83

6.3 Height Dependency: A More Detailed Study . . . . . . . . . . . . . . 84

6.3 .1 Creeping Sedimentation .................. 85

6.4 Height and Width Dependency . . . . . . . . . . . . 87

6.4.1 Experimental Method ................ 87

6.4 .2 Results ........................... 88

6.4 .3 Some Observations . . . . . . . . . . . . . . . . . . 91

6.4 .4 A Theoretical Explanation $\ldots \ldots . \ldots . \ldots . \ldots 92$ 
7 Discussion

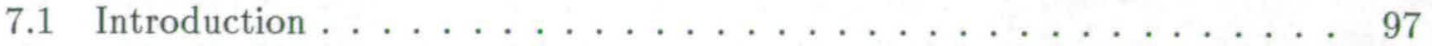

7.2 Initial Structure of Transient Gels f . . . . . . . . . . . . . 98

7.3 Delayed Sedimentation . . . . . . . . . . . . . . . . . . . . 99

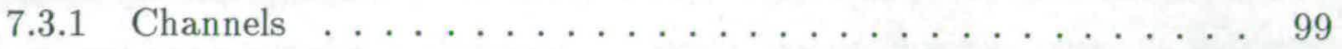

7.3 .2 Streamers ......................... 103

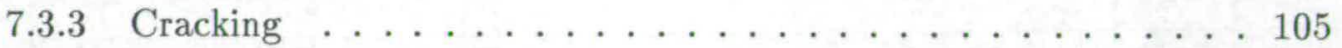

7.3 .4 Bright Bands . . . . . . . . . . . . . 106

7.4 Size Effects . . . . . . . . . . . . . . . . 106

7.5 Mechanisms for Restructuring of Transient Gels . . . . . . . . . . . . 107

7.5.1 Thermally Activated Coarsening and Sintering . . . . . . . 107

7.5 .2 Fluid Instabilities . . . . . . . . . . . . . . . 110

7.5.3 Weight-Induced Coarsening . . . . . . . . . . . . 111

7.6 A Qualitative Picture of Delayed Sedimentation in Transient Gels . . . . 112

7.7 Crossover from Delayed to Creeping Sedimentation . . . . . . . . . . . 113

7.8 Suggestions for Future Work f . . . . . . . . . . . . . . . . . 114

8 Conclusion

List of Figures

List of Tables 


\section{Chapter 1}

\section{Introduction}

\subsection{What is a Colloid ?}

A colloid is formed by suspending small parts of one material, the dispersed phase, in a continuum of another. The defining feature of a colloidal suspension is the size of the dispersed components which lie within the mesoscopic regime, between the nanometre and micrometre scales. This means that the constituent parts of the dispersed phase are large enough to be treated in a purely classical way but small enough such that Brownian motion will dominate over gravitational settling. In this particular work we shall be concerned with suspensions of solid particles in a liquid continuum. However, it should be noted that other types of colloidal suspensions exist, e.g. liquid-in-liquid suspensions known as emulsions are studied in great detail within the food industry.

\subsection{Why Study Colloids ?}

Colloidal materials are commonly found in everyday substances such as paint, toothpaste, crude oil, and many food products like milk and mayonnaise. It is not surprising then to find that research in colloidal systems spans many branches of science and engineering or that much of modern colloid research occurs in industrial laboratories. For example, one interesting field in colloid science is to understand the formation of metastable states and the way in which the system reaches equilibrium. This kind of 
work has many applications as a lot of commercial products are only useful in their non-equilibrium state.

Another interesting aspect of studying colloidal materials is that they can provide useful insight into the physics of atomic systems. The equilibrium thermodynamic properties of a suspension of colloids are formally equivalent to those of an assembly of atoms with the same interparticle potential. Under suitable conditions we can find phase behaviour in colloidal systems analogous to that of simple atomic systems such as argon. For example, the study of the early stages of crystal nucleation is impossible to do with atomic systems due to the very short time-scales involved in the process. However, crystallisation in colloidal materials takes place over many hours or even days and can be observed in an optical microscope [1].

Finally, colloidal materials are interesting in their own right exhibiting phenomena not observed in any other systems.

\subsection{Hard-Sphere Colloids}

Real colloidal systems are incredibly complex often involving many non-identical components. In order to come to a more fundamental understanding of these systems we appeal to model systems which are much less complicated though not necessarily simple to understand.

The simplest theoretical model which we shall study is a suspension of identical hardspheres. In this model the particles have no interaction with each other until they come within touching distance where they experience an infinite repulsion. Hard-spheres systems have no energy scale thus we need only consider entropic effects when determining the phase behaviour [2]. We can thus characterise these systems by the number density of particles alone. This is more commonly expressed as the colloid volume fraction $\phi$ which for a collection of $N$ spheres of radius $a$ in a system volume $V$ is given by

$$
\phi=\frac{4}{3} \pi a^{3} \frac{N}{V}
$$

The phase behaviour of an arrangement of single-sized hard-spheres has been predicted 
by computer simulation $[3,4]$ and is shown schematically in figure 1.1 . Only the volume fraction axis is shown as altering the temperature has no effect on the phase behaviour. Below a freezing volume fraction of 0.494 a colloidal fluid is observed. Each particle is able to explore all of the available volume through diffusion and at any one instant the arrangement of particles will show only short ranged order.

At volume fractions above $\phi=0.494$ but below $\phi=0.545$ the system phase separates into coexisting fluid and crystalline phases of volume fractions $\phi_{F}=0.494$ and $\phi_{M}=$ 0.545 respectively. Observation of samples in this state show homogeneous nucleation of small iridescent crystallites which then sediment to form a polycrystalline phase at the bottom of the sample cell. As the volume fraction is increased further more crystals form at the expense of the fluid phase until, at the melting volume fraction $\left(\phi_{M}=0.545\right)$, the system becomes fully crystalline. Further increasing the volume fraction results in producing a more compact crystalline arrangement up to a maximum close packing volume fraction of $\phi_{c p}=0.74$. However, if the particles adopt a random close packed arrangement then the maximum volume fraction obtainable will be $\phi_{R C P}=0.64$.

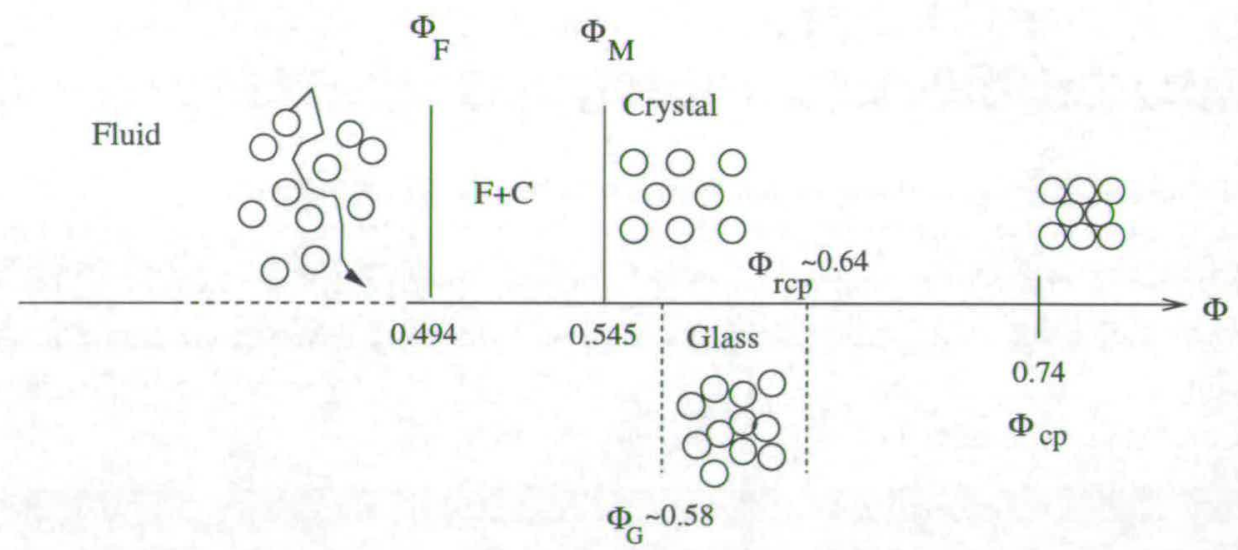

Figure 1.1: Phase behaviour of a suspension of monodispersed hard-spheres.

Hard-sphere systems also exhibit non-equilibrium behaviour. At $\phi_{G} \approx 0.58$ crystallisation is suppressed by the formation of an amorphous solid known as a glass. The transition to the glassy state is thought to be due to the suppression of long-time diffusion above the glass transition volume fraction [2].

How is it possible for a hard-sphere system to form an ordered state when only entropy considerations determine the phase behaviour? The answer lies in the competition 
between configuration and free volume entropy [2] as illustrated in figure 1.2. This figure shows two ways in which eight identical discs can be packed into a box. In figure 1.2 (A) the discs are packed in a random arrangement which has a high configurational entropy. However, each disc is restricted in its movement so that the free volume entropy is low. Figure 1.2 (B) shows the opposite example in which the discs are arranged in a highly ordered state. Although this second arrangement has low configurational entropy it has high free volume entropy as each particle is now free to move about its average position. Crystallisation of a suspension of hard-sphere occurs when the entropy gained by creating more free volume for each particle exceeds that lost by having an ordered arrangement.

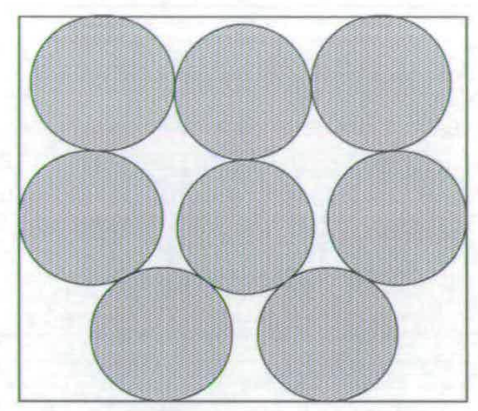

(A)

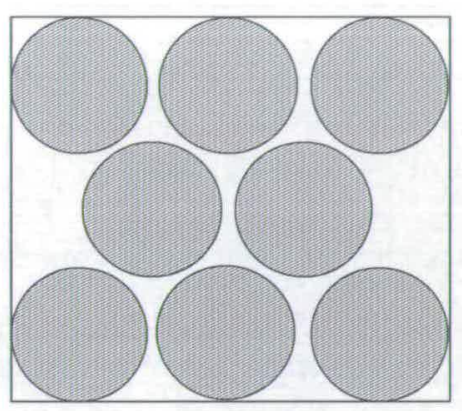

(B)

Figure 1.2: Schematic diagram of two dimensional hard discs in a box showing how competition between configurational and free volume entropy can drive freezing. $(A)$ a random configuration of discs has a high configurational entropy but low free volume entropy. (B) a crystalline arrangements of discs with low configurational entropy but high free volume entropy due to an increase in the local mobility of each disc.

\subsubsection{Building a Model Hard Sphere System}

Ideal suspensions of identical hard-spheres exist only in theory. However, real systems can be synthesised which reproduce the behaviour of the model hard-sphere system extremely well. Such a system provides the main ingredient for the colloid-polymer mixtures work to be presented in this thesis. First of all we consider some important issues in creating model systems for experiments. 


\section{Instability of Colloidal Suspensions}

Many colloidal systems are unstable to aggregation due to van der Waals attractions between the particles. Two neighbouring atoms or molecules can attract each other due to fluctuations in their dipole moments [5]. This is true even for neutral atoms and molecules. Although quantum mechanical in origin, van der Waals forces can be long ranged enough to create attraction between the atoms of two macroscopic bodies.

In the limit of small separations, the van der Waals interaction energy between two identical macroscopic spheres of radius $R$ is given by

$$
U_{V d W}(D)=-\frac{A R}{12 D}
$$

where $A$ is known as the Hamaker constant and $D$ is the separation between the surfaces of the two spheres. The van der Waals interaction results in an attractive potential minimum near the surface of the particles which can be many times $k_{B} T$ in magnitude. Thus in the absence of any stabilising interactions the particles will aggregate.

The Hamaker constant $A$ depends upon the frequency dependent polarisability of the particles and the suspending medium. If the particles and the suspending medium have the same frequency dependent polarisabilities then the Hamaker constant will be zero and there will be no van der Waals attraction between the particles. The two media having the same polarisabilities is equivalent to them having equal refractive indices. Thus by matching the refractive index of the suspending solvent to that of the colloidal particles we can switch off aggregation due to van der Waals interactions at one particular frequency.

\section{Stabilisation against Aggregation}

It is necessary to stabilise our colloidal particles to prevent aggregation due to van der Waals interactions. There are two common approaches to particle stabilisation which depend on the nature of the suspension to be stabilised.

Some colloidal particles have ionisable groups on their surfaces which can dissociate 
from the particle when it is suspended in a polar solvent, e.g. water. The particles thus obtain a charge and can be thought of as macroions. The counterions discharged into the polar solvent diffuse away from the macroion but remain within the influence of the particle's electric field. Thus an electrical double layer of ions and counterions forms around each particle. When two particles come close to each other overlap of the electric double layers causes a repulsive force which can stabilise the suspension against aggregation. This is known as charge stabilisation.

Steric stabilisation is used when colloidal particles are dispersed in a non-polar solvent. In this case a thin layer of polymer molecules is chemically grafted onto the surface of each colloidal particle. As two particles approach each other, compression of the polymer layers produces a strong repulsion.

Sterically-stabilised colloids can approximate the behaviour of hard-spheres when the stabilising layer is very thin in comparison with the particle radius, for a good example with illustrative photographs see Pusey and van Megen [6].

\section{Size Polydispersity}

A suspension of identical particles is difficult to achieve in practice. In any colloidal system, even model systems, we almost always find a distribution in the size of the particles. The suspension is then said to be polydispersed. Size polydispersity is defined as the standard deviation in the size distribution divided by the mean value and is usually denoted by $\sigma$. Colloidal suspensions can also be polydispersed in other particle attributes such as shape, charge and density.

Polydispersity is an important issue as even small amounts can affect the physical properties of a suspension [7]. However, theoretical studies often ignore polydispersity as it is a difficult parameter to include. Therefore, it is necessary to synthesise colloidal systems with very low polydispersity such that we may approximate them to monodispersed systems. 


\subsubsection{Our Model System}

The colloidal suspensions used in this study consist of polymethylmethacrylate (PMMA) spheres dispersed in an organic non-polar solvent, typically cis-decahydronapthaline (cis-decalin). The particles are approximately $200 \mathrm{~nm}$ in radius and are sterically stabilised by chemically grafting a thin layer $(\approx 10 \mathrm{~nm})$ of poly-12-hydroxystearic acid (PHSA) to the surface. The polydispersity of the particles in this system varies with their mean size. The smallest particles are the most polydispersed with up to a $20 \%$ variation in size. However, the polydispersity for particles with radii $a \geq 150 \mathrm{~nm}$ can be as low as $4 \%$ or $5 \%$.

\subsection{Thesis Summary}

This thesis presents an experimental study of the collapse of transient colloid-polymer gels. The initial motivation for this work was to further our understanding of delayed sedimentation by exploring the effects of shear and polydispersity on gel collapse. However, it was soon clear that the picture of gel collapse in monodispersed systems without shear was far from complete and that more observations were required in order to fill in the gaps. This thesis presents the results of those observations.

Moreover, each of these observations merely served to reveal previously unknown aspects of the gels. Therefore, the results in this thesis, taken together, should be seen as defining the transient gel collapse problem for the first time. Only a very tentative solution, in the form of a speculative picture of the collapse process, will be presented.

In this first chapter, some background on colloidal suspensions of hard-spheres has already been introduced. This is built upon in chapter 2 where some background on colloid-polymer mixtures is presented. The discussion of colloid-polymer mixtures will concentrate on the behaviour of non-equilibrium states, especially the transient gel. The main results of a previous study of gel collapse are also presented.

Some essential technical details of the two main techniques used to study gel collapse in this work, dark-field imaging and ultrasonic concentration profiling, are introduced in chapter 3. 
In chapter 4 the results of a dark-field observation study of gel collapse are presented and discussed. Chapter 5 is concerned with the evolution of gel concentration during the collapse process. Chapter 6 focuses on the size dependency of delay time where we compare experimental data with a recent theory.

Chapter 7 attempts to pull together the results of this work and other related studies reported in current literature in order to form a consistent explanation of gel collapse. Some suggestions of future work are also made.

Finally, chapter 8 presents some basic conclusions which have been made based upon this work. 


\section{Chapter 2}

\section{Colloid-Polymer Mixtures}

\subsection{Introduction}

Colloid-polymer mixtures exhibit a wide range of equilibrium and non-equilibrium behaviour which are of fundamental scientific interest. Such systems are also of interest to industrial research as they are common in many commercial products. In this chapter we introduce some theoretical background on colloid-polymer mixtures which focuses on the non-equilibrium behaviour of such systems. An overview of transient gel collapse is also included.

\subsection{The Depletion Potential}

The addition of non-adsorbing polymer to a suspension of colloidal hard-spheres can cause phase separation via the depletion mechanism. A theoretical model of depletion was first suggested by Asakura and Oosawa [8] in 1958, and independently by Vrij [9] in 1976. Polymer coils and colloidal particles are mutually impenetrable and thus the polymer centres of mass are excluded from a region around each colloidal particle of order $r_{g}$, the polymer radius of gyration, see figure 2.1 . The polymer coils are restricted to the volume not occupied by colloidal particles and their depletion zones, the free volume $V_{\text {free }}$. The overlap of depletion layers of nearby particles creates more free volume for the polymer coils and thus lowers the free energy by maximising entropy. In this way, an effective attraction between the particles is induced. 


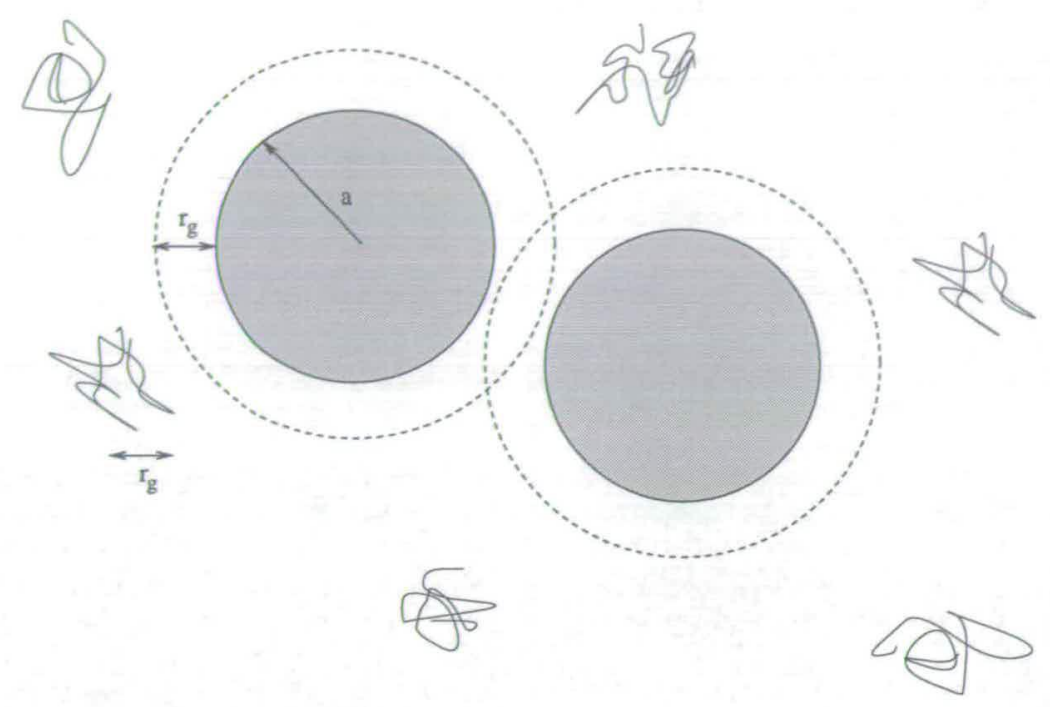

Figure 2.1: Diagram showing the depletion zones of two colloidal particles, radius $a$, overlapping and how random coil polymers, radius of gyration $r_{g}$, are depleted from the region between the particles.

The attraction between the particles can be modelled by a pair potential, $U(r)$ given by

$$
U(r)=\left\{\begin{array}{ll}
+\infty & r \leq \delta \\
-\Pi_{p} V_{\text {overlap }}=U_{\text {dep }} & \delta<r \leq\left(\delta+2 r_{g}\right) \\
0 & r>\left(\delta+2 r_{g}\right)
\end{array}\right\}
$$

where $\delta=2 a$ is the particle diameter, $r_{g}$ is the polymer radius of gyration, and $\Pi_{p}$ is the osmotic pressure exerted by the polymer solution on the particles. $V_{\text {overlap }}$ is the volume of the overlapping depletion layers between two particles at intercentre separation $\mathrm{r}$, which is given by

$$
V_{\text {overlap }}=\left(1-\frac{3 r}{2 \delta(1+\xi)}+\frac{1}{2}\left[\frac{r}{\delta(1+\xi)}\right]^{3}\right) \frac{\pi}{6} \delta^{3}(1+\xi)^{3}
$$

where $\xi=\frac{r_{g}}{a}$ is the polymer to colloid size ratio.

For a dilute suspension of polymers, i.e. one with polymer volume fraction $\phi_{p}$ well below coil overlap volume fraction, at the theta temperature $T_{\theta}$ we can assume that 
the coils interact as an ideal gas. The osmotic pressure is then given by van't Hoff's Law

$$
\Pi_{p}=\frac{N_{p}\left(k_{B} T\right)}{V_{\text {free }}}
$$

where $N_{p}$ is the number of polymer coils and $V_{\text {free }}$ is the free volume available to the polymer coils which is defined as

$$
V_{\text {free }}=V-\left(V_{c}+V_{\text {dep }}\right)+V_{o l} \quad,
$$

where $V$ is the total volume of the sample, $V_{c}$ and $V_{d e p}$ are the volume occupied by the colloidal particles and their depletion zones respectively. $V_{o l}$ is the total volume of the overlapping depletion layers.

The free volume in a suspension of particles $V_{\text {free }}\left\{r_{c}\right\}$ depends upon the positions of all the particles, $\left\{r_{c}\right\}$. In practice it is difficult to measure the free volume. We therefore use the total volume $\mathrm{V}$ which is related to the free volume by

$$
V_{\text {free }}=\alpha\left(\left\{r_{c}\right\}\right) V \quad
$$

where $\alpha\left(\left\{r_{c}\right\}\right)$ is known as the free volume fraction. In a simple mean field approximation, where free volume can be approximated by its average value in a corresponding unperturbed system of colloidal particles, $\alpha$ depends solely upon the colloid volume fraction.

An approximate form for $\alpha$ may be obtained from scaled particle theory $[10,11]$,

$$
\alpha=(1-\phi) \exp \left[-A \gamma-B \gamma^{2}-C \gamma^{3}\right] \quad,
$$

where $\gamma=\phi /(1-\phi), \mathrm{A}=3 \xi+3 \xi^{2}+\xi^{3}, \mathrm{~B}=\left(9 \xi^{2}\right) /\left(2+3 \xi^{3}\right)$ and $\mathrm{C}=3 \xi^{3}$.

Experimentally we characterise colloid-polymer mixtures by their colloid volume fraction, $\phi$, and the polymer mass concentration in the total volume, $C_{p}$. We can link the 
osmotic pressure $\Pi_{p}$, and so the depletion potential strength $U_{\text {dep }}$, to the experimental parameter $C_{p}$ by rewriting equation 2.3 as

$$
\frac{\Pi_{p}}{k_{B} T}=\left(\frac{N_{A}}{M_{w}}\right)\left(\frac{M_{p}}{\alpha V}\right)=\left(\frac{N_{A}}{M_{w}}\right) C_{p}^{f r e e},
$$

where $N_{A}$ is Avagadro's number, $M_{w}$ is the molecular mass of the polymer, $M_{p}$ is the total mass of polymer in the system and $C_{p}^{f r e e}$ is the mass concentration of polymer in the free volume. $C_{p}^{f r e e}$ is defined as

$$
C_{p}^{\text {free }}=\left(\frac{M_{p}}{\alpha V}\right)=\frac{C_{p}}{\alpha}
$$

In equation $2.8, C_{p}$ is the experimentally accessible polymer mass concentration in the total volume. From this we can see that $C_{p}$ is proportional to $\Pi_{p}$ and thus to the depletion strength $U_{\text {dep }}$. Thus the more polymer that is added to a colloidal suspension the stronger the depletion attraction induced will be.

\subsection{Predicting the Phase Behaviour}

To determine the phase behaviour of a colloid-polymer mixture we must find a way to minimise its Helmholtz free energy, $F$. Here we shall consider the method of Lekkerkerker et al. [12]. In this approach the free energy density, $f=F / V$, of a colloid-polymer mixture is written as a function of the colloid volume fraction, $\phi$, and the chemical potential of the polymer, $\mu_{p}$. At fixed temperature, $\mu_{p}$ is a function only of the number

density of polymers in the free volume, $n_{p}^{f r e e}=N_{p} / V_{\text {free }}$, which in turn is related to the free volume polymer concentration. Thus $f=f\left(\phi, C_{p}^{f r e e}\right)$.

Using a mean field approach, the free energy density for the system can be calculated for two distinct arrangements of polymers and colloids: a disordered arrangement of colloids and polymers, giving the "fluid branch" and an ordered arrangement of colloids with polymers randomly distributed, giving the "crystal branch". The free energy density can thus be calculated for any fixed depletion strength, determined by $C_{p}^{f r e e}$, and plotted against colloid volume fraction. 
Figure 2.2 shows schematically the form of the free energy density, $f$, with volume fraction, $\phi$, for a low pair-potential strength, i.e. low $C_{p}^{f r e e}$. To determine the concentrations of any coexisting phases we use the common tangent construction [13] as shown in the figure. This is a geometrical method to find the solutions of the free energy density which satisfy the conditions for equilibrium, namely that the polymer chemical potential and the pressures of the two coexisting phases are equal.

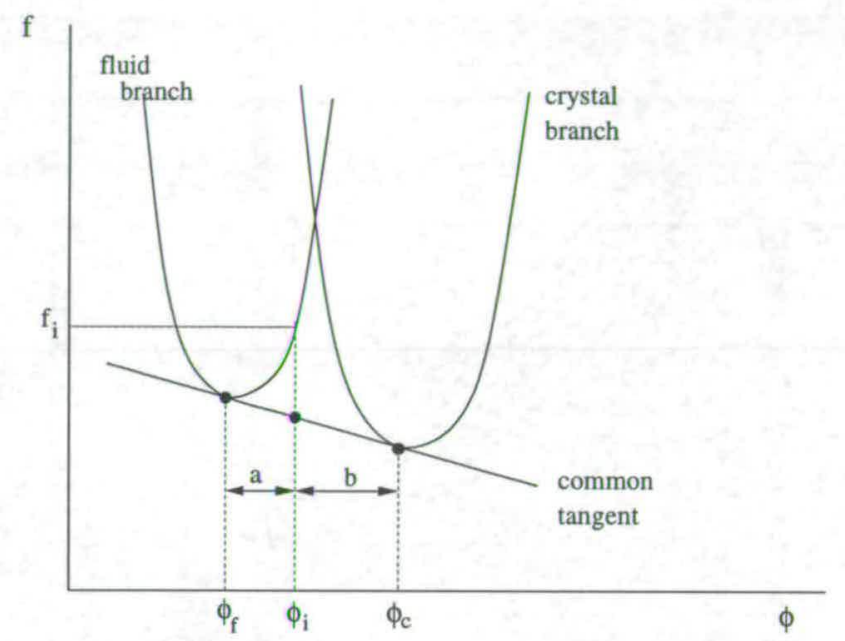

Figure 2.2: Schematic of the free energy density, $f$, as a function of colloid volume fraction, $\phi$, for fixed free-volume polymer concentration $C_{p}^{\text {free }}$. This figure shows how the common tangent construction may be used to determine the concentrations of the two coexisting phases; the lever rule giving the amounts of each phase we expect to find.

Using this method we can see from figure 2.2 that an initially homogeneous fluid with concentration $\phi_{i}$ can lower its free energy density $f_{i}$ by separating into a coexisting fluid phase with concentration $\phi_{f}$ and crystal phase of concentration $\phi_{c}$. The relative volumes of the two phases are found from the lever rule [13] $\left(V_{f} / V_{c}=b / a\right)$.

To build up a complete phase diagram this procedure is repeated for a range of depletion depths, i.e. $C_{p}^{f r e e}$. In this way we can predict the phase behaviour as a function of colloid volume fraction $\phi$ and free polymer concentration $C_{p}^{f r e e}$.

It is found that the predicted phase behaviour depends on the range of the potential, i.e. $\xi=r_{g} / a$, as well as on volume fraction and polymer concentration. When the size ratio is less than $\sim 0.3$ the only effect of adding polymer is to expand the region of fluid-crystal coexistence observed in the pure hard-sphere colloid. With larger size ratios a whole range of phase behaviour becomes possible. A critical point can be 
observed and distinct gas and liquid phases are predicted. Also, regions of three phase coexistence are possible.

In this study we shall only be concerned with small size ratio systems, i.e. $\xi \approx 0.1$. Figure 2.3 shows a typical predicted phase diagram for a colloid-polymer system with size ratio $\xi=0.08$ in terms of $C_{p}$ and $\phi[14]$. Oblique tie-lines in the two phase region imply polymer partitioning between the fluid and crystal phases.

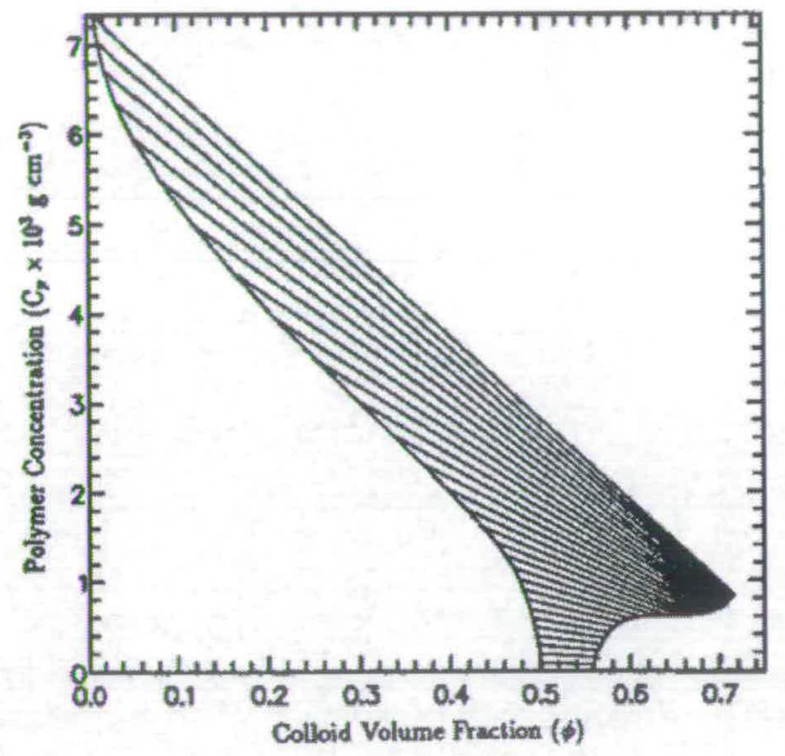

Figure 2.3: Predicted phase diagram, polymer concentration $C_{p}$ against colloid volume fraction $\phi$ for a system where $\xi=0.08$. Oblique tie-lines in the two phase region imply polymer partitioning between the fluid and crystal phases. Figure reproduced from [14].

\subsection{Observed Equilibrium Phase Behaviour}

In practice we can explore the phase behaviour of a colloid-polymer system by varying $\xi$, the colloid volume fraction $\phi$ and the polymer concentration $C_{p}$. Figure 2.4 shows an experimentally determined phase diagram for a typical (PMMA-polystyrene) colloidpolymer system suspended in cis-decalin [14]. The particle radius was $a=217 \pm 5$ $\mathrm{nm}$ with a polydispersity of around $5 \%$. The polymer used was of molecular weight $M_{w}=390,000$. The observations were made at room temperature $\left(T=19 \pm 2^{\circ} \mathrm{C}\right)$. The radius of gyration for the polymer at this temperature was $19 \mathrm{~nm}$ giving a size ratio $\xi \approx 0.09$. 


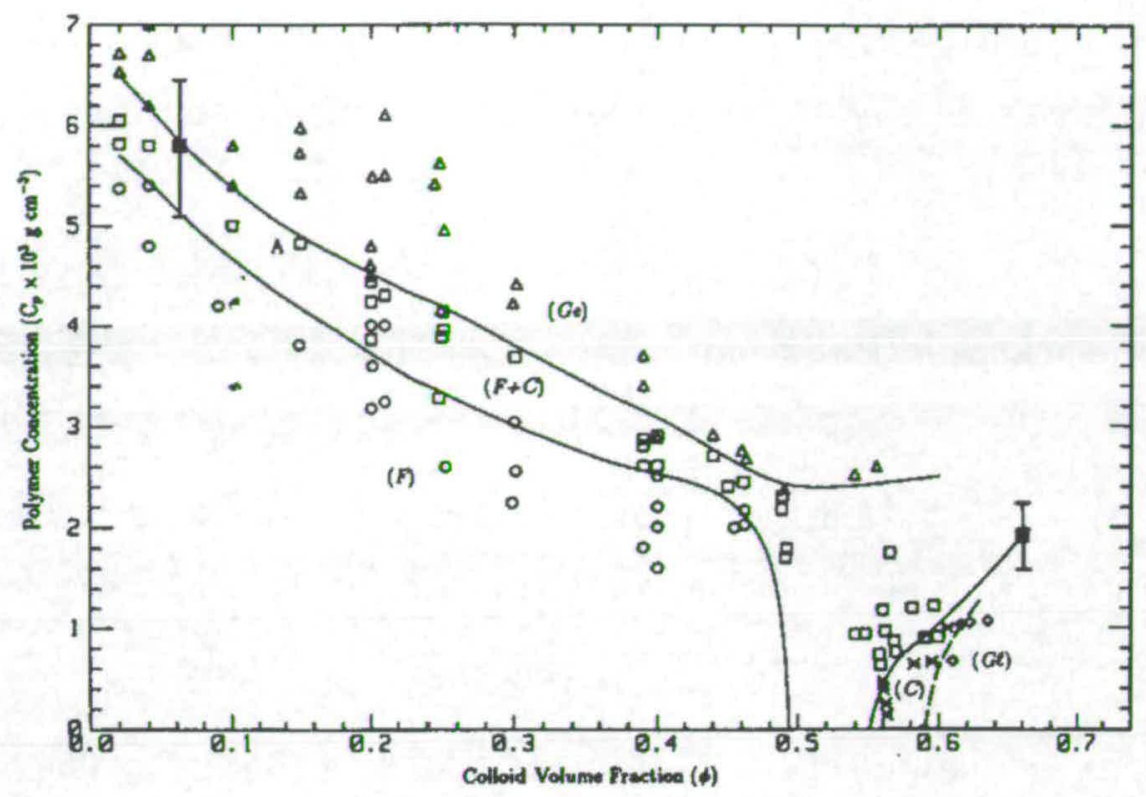

Figure 2.4: Experimental phase diagram $\left(C_{p}\right.$ against $\left.\phi\right)$ for a PMMA-PS colloid-polymer mixture of size ratio $\xi \approx 0.09$. (०) homogeneous colloidal fluid, $(\square)$ fluid-crystal coexisting, $(\triangle)$ non-equilibrium aggregating state, $(x)$ fully crystalline, and $(\diamond)$ glass. Solid lines are drawn to act as guides to the eye in identifying the position of phase boundaries. Figure reproduced from [14].

When a small amount of polymer is added (typically less than $0.5 \mathrm{mg} / \mathrm{cm}^{3}$ ) no significant differences in the observed phase behaviour from that of a hard-sphere colloidal suspension, as shown in figure 1.1, are observed. A fluid-like phase (open circles figure 2.4 ) is observed up to a volume fraction of $\phi=0.494$ above which coexisting fluid and crystals phases are observed (open squares in figure 2.4). Above a volume fraction of $\phi=0.545$ the system becomes fully crystalline, indicated by crosses in the above figure. At higher volume fraction, $(\phi \approx 0.58)$, crystallisation is suppressed and a glass-like arrangement of particles is found.

Upon adding more polymer to the system it is found that the region of fluid-crystal coexistence is expanded as predicted by theory. However, at higher polymer concentrations crystallisation is suppressed and a sequence of non-equilibrium behaviour is observed.

It should be noted that the concentration of polymer required to observe these nonequilibrium behaviours $\left(C_{p} \gtrsim 4.60 \mathrm{mg} / \mathrm{cm}^{3}\right.$ at $\left.\phi=0.2\right)$ is much lower than the overlap 
concentration of the polymer coils $\left(C^{*} \simeq \frac{M_{w}}{N_{A}} \frac{3}{4 \pi r_{g}^{3}}=22.5 \mathrm{mg} / \mathrm{cm}^{3}\right)$. Thus the suppression of crystallisation cannot be attributed to a change in the behaviour of the polymer.

\subsection{Non-equilibrium Behaviour}

In the previous section we presented some experimental evidence for the suppression of crystallisation at high enough polymer concentration. We shall now discuss nonequilibrium behaviour in colloid-polymer mixtures in more detail.

An experimental study using small angle light scattering (SALS) by Poon at al.[15] shows evidence for three different types of non-equilibrium behaviour in a small size ratio system, $\xi \approx 0.08$. This is summarised in the diagram shown in figure 2.5 below.

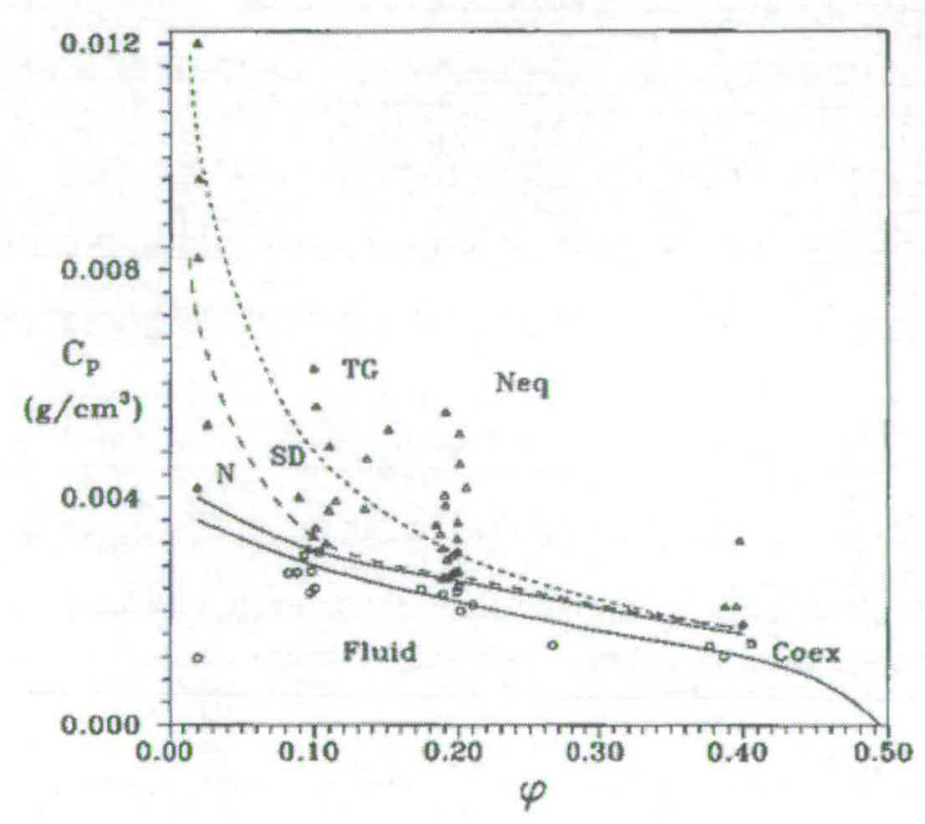

Figure 2.5: Phase diagram expanded to include non-equilibrium "phases" for a colloid polymer mixture with size ratio $\xi \approx 0.08$. Neq $=$ non-equilibrium, TG $=$ transient gel, $\mathrm{SD}$ = spinodal-like, $\mathrm{N}=$ nucleation-like. The marked boundaries are: fluid-crystal coexistence (lower solid line), non-equilibrium boundary (upper solid line), nucleation-spinodal boundary (long dashes) and transient gelation boundary (short dashes). Figure reproduced from [16]. 


\section{Nucleation (N)}

At low volume fraction and just over the non-equilibrium boundary, a ring of scattered light appears at low angles after an initial delay. The ring then brightens and collapses, shrinks in diameter, with time. Observations under the microscope show the formation of disconnected aggregates which are reminiscent of nucleation of droplets in classical fluids. This behaviour is thus labelled as nucleation-like (N-type).

\section{Spinodal Decomposition (SD)}

Just above the non-equilibrium boundary and at moderate $\phi$ the small angle ring appears immediately. The collapse of the ring is continuous and complete. Dynamic scaling of the scattering intensity at intermediate time is similar to that of classical spinodal decomposition in fluids. Observations under the microscope show the formation of interconnected domains. This behaviour is labelled as spinodal-like (S-Type).

\section{Transient Gelation (TG)}

A third type of non-equilibrium behaviour, Transient-Gelation (T-type), is observed at higher polymer concentrations than the previous two. Here the collapse of the ring appears frozen after a small initial collapse. Temporal fluctuations in the scattering pattern are very slow and appear to the naked eye to be non-existent. After a period of time the fluctuations speed up and the ring collapses within seconds to give a peaked forward scattering typical of an amorphous sediment.

Dynamic scaling of the scattering intensity at intermediate times shows a characteristic scaling exponent of 1.7. This is consistent with the presence of clusters formed by diffusion limited cluster aggregation (DLCA) [17].

\subsubsection{Onset of Non-equilibrium Behaviour}

It has been proposed by Poon et al. [15] that the crossover from equilibrium to nonequilibrium behaviour is due to aggregation of the colloidal particles. 


\section{A Model for Aggregation}

A simple model which appears appropriate to describe our system is Diffusion Limited Cluster Aggregation (DLCA) [17]. In this model particles are assumed to be strongly attracting each other. The particles are allowed to explore space by diffusion but upon touching they form permanent interparticle bonds. Thus clusters of particles are formed which continue to diffuse. When two clusters meet they bond to form a larger cluster.

Clusters formed by DLCA have a fractal structure, i.e. the number of particles in a cluster ( $\propto$ mass of particles) scales with cluster radius to some power $d$ (known as the fractal dimension) which is smaller than the spatial dimension $D$. In three dimensions $(D=3)$ DLCA produces a fractal dimension $d \approx 1.8$.

As a cluster grows by DLCA, it becomes more tenuous and so its average density (or average volume fraction) decreases, see figure 2.6. Gelation in this model occurs when the average volume fraction of each cluster is the same as the total sample volume fraction, i.e. all the clusters touch and span the sample.

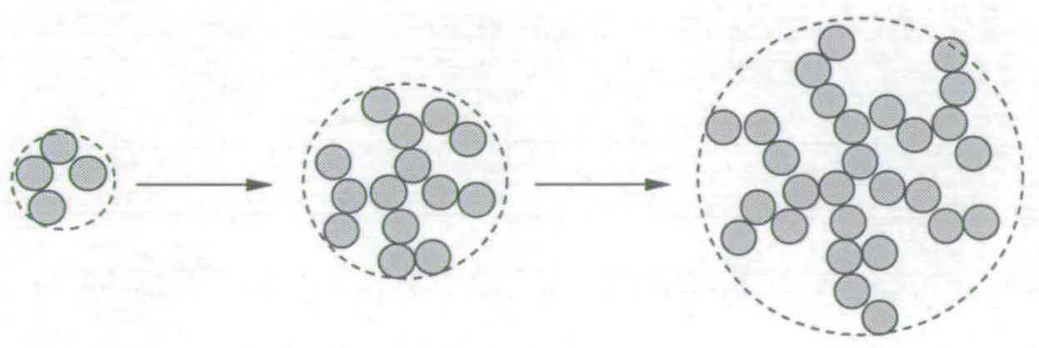

Figure 2.6: Schematic diagram showing the growth of a fractal cluster. As the cluster grows larger its structure becomes more tenuous and its average density decreases

\section{Aggregation vs Thermal Rearrangement}

The DLCA model describes systems with very strong interparticle attractions. We are interested in weakly aggregating systems in which interparticle bonds can be broken by the thermal energy of the particles, $\sim k_{B} T$. In this case particle aggregation and thermal fluctuations compete. This can be modelled by DLCA with finite bond energies known as "reversible DLCA" [18]. 
In reversible DLCA the particles still bond upon touching but now there exists a finite probability that the bond can be broken. The probability of bond breaking follows a Boltzmann distribution, $\sim \exp \left(-U / k_{B} T\right)$.

Figure 2.7 shows three "snap-shots" of particle aggregation produced from a $2 \mathrm{D}$ reversible DLCA simulation by Haw et al. [19]. In each image black spots indicate the position of colloidal particles.

Figure 2.7(A) shows the formation of a gel network when $U_{d e p} \gg k_{B} T$, i.e. irreversible DLCA. The network is formed of single particle links. This is the kind of structure we may expect to see in the absence of gravity and strong interparticle attractions.

For lower bond strengths, figure 2.7(B), thermal fluctuations can cause compaction of the clusters on small length scales. However, the clusters can still grow fast enough to span the system. The arms of the gel network are made up from double or even triple bonded particles making them fatter than in the previous case. As time progresses the structure is seen to break into two or three large clusters and then reform due to thermal motion of the particles. We also note the large channels formed between each arm of the network. In a real system these would be filled with solvent (and polymer). It is possible that in real systems thermal rearrangements coupled with fluid flow through the channels and gravity could lead to the collapse of these type of structures. Thus we identify this particular case with the transient gel.

At even lower bond energy, figure 2.7(C), compactification of clusters by thermal fluctuations wins over aggregation. The clusters formed cannot grow fast enough to form a space-spanning network. This case is analogous to the spinodal-like behaviour observed in real systems.

\subsubsection{Influence of Gravity on the Gel Boundary}

In the absence of gravity we can identify the crossover from the case of compact cluster formation to a space-spanning network with the transient gelation line. However, few of the systems under study in this work are free from gravitational effects and we would like to know how the position of the gel line may be affected by gravity. 


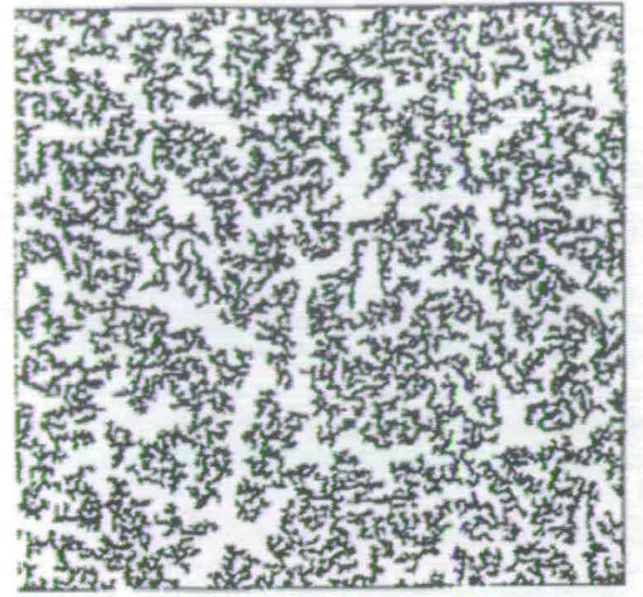

(A)

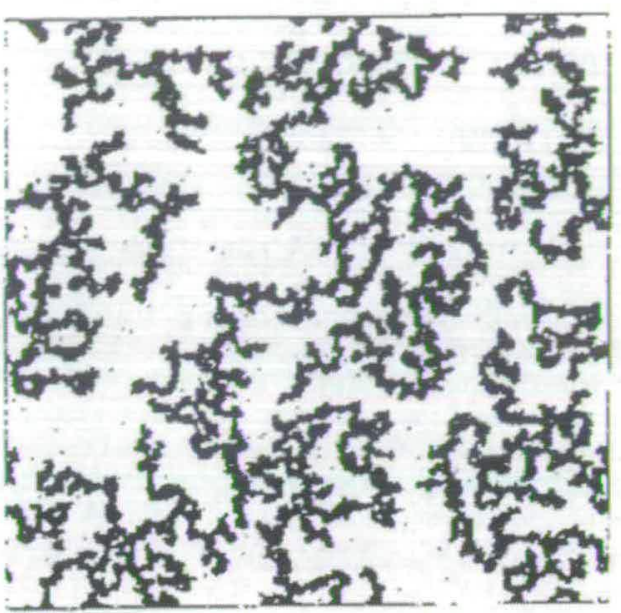

(B)

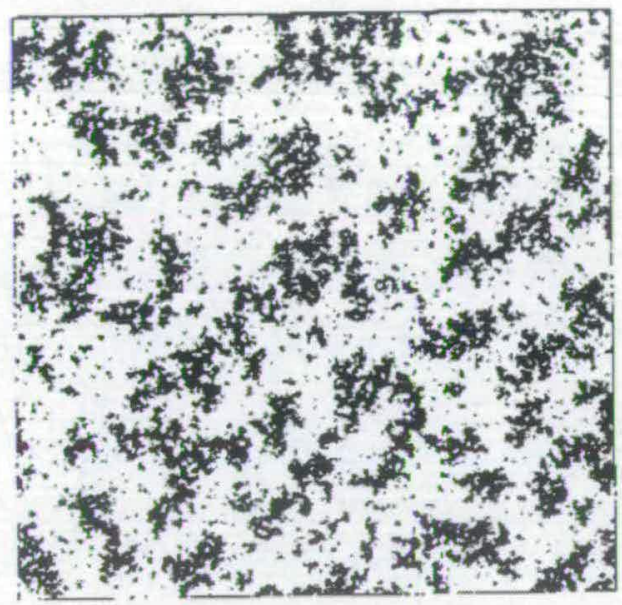

(C)

Figure 2.7: 2D Reversible DLCA simulation of particle aggregation with varying bond strength. (A) $U_{d e p} \gg k_{B} T$, (B) $U_{d e p} \sim 3 k_{B} T$, (C) $U_{d e p} \approx k_{B} T$. Figure reproduced from [19]

A simple model proposed by Poon [20] and Allain [21] attempts to explain the effect of gravity on the position of the transient gel boundary. The model predicts a threshold volume fraction $\phi^{*}$ below which gelation does not occur. Below this threshold volume fraction, the clusters cannot diffuse fast enough to form a space-spanning network. At infinite interparticle bond energy, $\phi^{*}$ can be written as

$$
\phi^{*} \sim\left(\frac{9 k_{B} T}{2 \pi \Delta \rho g a^{4}}\right)^{\frac{d-3}{d+1}}
$$

where $\Delta \rho$ is the difference in density between the particles and the suspending solvent, 
$g$ is the gravitational acceleration constant, $a$ is the radius of the particles and $d$ is the fractal dimension of the clusters ( $d \approx 1.8$ for DLCA clusters).

The effect of increasing the influence of gravity in this model is to increase $\Delta \rho g$ thus increasing $\phi^{*}$. We therefore expect to see the onset of transient gelation at much higher volume fractions than those predicted by just considering aggregation-thermal fluctuation competition.

\subsubsection{The Sharpness of the Non-equilibrium Boundary}

The reversible DLCA model can explain the crossover to a non-equlibrium regime. However, it is unable to predict the position of the various non-equilibrium boundaries. The position of these boundaries in real systems are highly reproducible and sharply defined and any theoretical framework must be able to reproduce this property.

Poon has proposed a method for predicting the non-equilibrium boundary using a thermodynamic approach [15]. He suggests that non-equilibrium behaviour is switchedon by the presence of a hidden gas-liquid binodal within the fluid-crystal region of the phase diagram. The origin of this hidden binodal is in the double minimum found in the fluid branch of the free energy landscape at strong enough depletion potential, see figure 2.8 .

The points on the equilibrium phase boundary (fluid-crystal) for this polymer concentration are obtained by constructing the lowest common-tangent between the fluid and the crystal branches. However, we can draw another common tangent between the two minima of the fluid branch which produces two points on a gas-liquid binodal inside the equilibrium fluid-crystal binodal. It has been found that both the fluid-crystal binodal and hidden gas-liquid binodal agree, at least qualitatively, with the equlibrium and non-equilibrium boundaries found experimentally.

If the identification of the non-equilibrium boundary with the hidden gas-liquid binodal is valid then we may expect to find behaviour similar to that of simple fluids undergoing phase separation just above the non-equilibrium boundary. For simple liquids we expect to see nucleation of droplets close to the binodal followed by spinodal decomposition further in. This kind of non-equlibrium is observed in colloid-polymer systems lending support to Poon's proposal. 


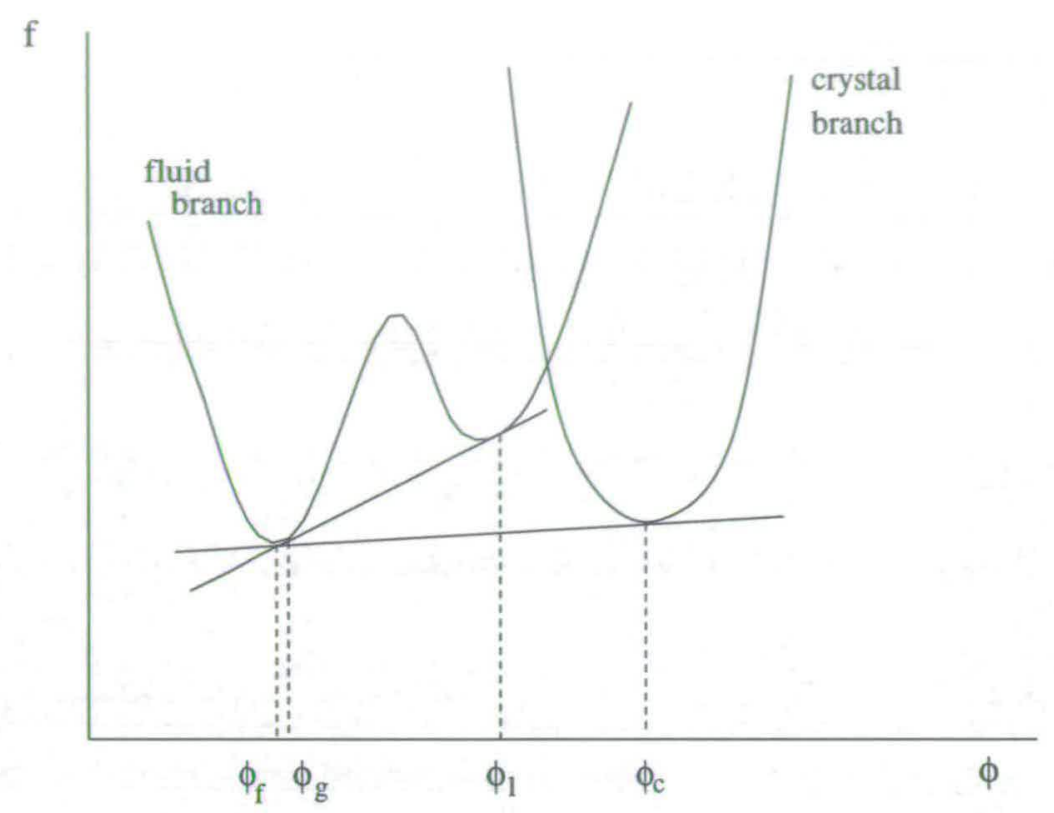

Figure 2.8: Schematic diagram of the free energy density against volume fraction of colloid where the pair-potential, i.e. $C_{p}^{f r e e}$, is large.

\subsection{Transient Gel Collapse}

In the previous section we used the idea of reversible DLCA to explain the formation of transient gels in colloid-polymer systems. We now turn to the very different problem of gel collapse. In this section we shall introduce the findings of a previous study [16] into gel collapse which has posed many questions that this current study has attempted to answer.

\subsubsection{Delayed Sedimentation}

Direct observation of transient gels show that they display delayed sedimentation. This is an interesting non-equilibrium phenomenon which is observed in many weakly flocculated systems, e.g. polymer-stabilised oil-water emulsions [22], aqueous suspensions of calcium carbonate [23], and suspensions of needle-like particles of iron oxide [24]. The defining characteristic of this behaviour is the presence of a delay time, during which little or no settling of the suspension is observed, before a sudden onset of rapid sedimentation. Delayed sedimentation is a general behaviour of the suspension which is not yet fully understood. 
Delayed sedimentation occurs in three stages as shown in figure 2.9. Firstly, a period of slow settling of the suspension is observed, (region A). The end of the slow sedimentation period is followed by a sudden increase in the sedimentation rate (region B). The point at which this occurs defines the delay time of the suspension $(\tau)$. This delay time can vary from a period of hours to months depending on the conditions of the system under study. During the rapid collapse a dense amorphous sediment forms at the bottom. The final stage of the settling process is the slow compaction of this sediment (region C).

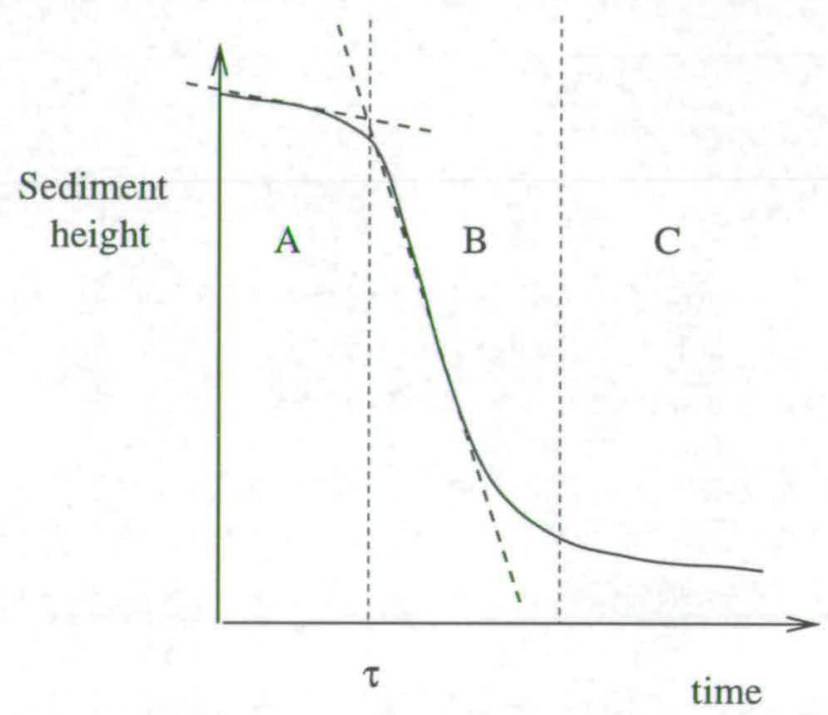

Figure 2.9: Schematic diagram showing the typical sedimentation profile of a suspension exhibiting delayed sedimentation. Regions $A, B$ and $C$ represent the three phases of the settling process. $\tau$ is the delay time.

The settling rates in the first two stages of delayed sedimentation are approximately linear. It is therefore possible to extract settling velocities for these two regimes from the sedimentation curve by fitting average lines to the data.

We can correlate the SALS ring behaviour to the settling behaviour as shown in figure 2.10. After initially shrinking in size during the first few minutes, the scattering ring remains frozen throughout the remainder of the delay stage. The point at which the ring collapses is closely correlated in time with the onset of rapid sedimentation. The time at which the small angle ring collapses is therefore a measure of the delay time.

The gel appears to be in a quiescent state throughout the delay period and we are given 


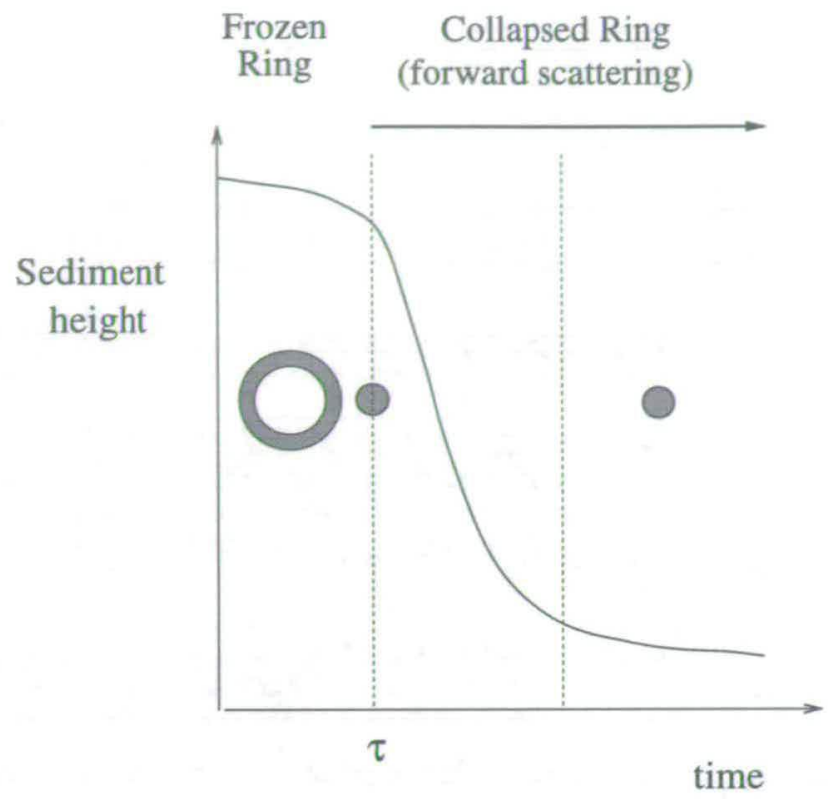

Figure 2.10: Schematic diagram showing how the characteristic profile of delayed sedimentation corresponds to the small angle light scattering ring of a transient gel. The forward scattering peak is typical of an amorphous sediment.

no clue either by observations or SALS to indicate that a gel is about to collapse.

\subsubsection{Predicting the Delay Time}

The formulation of a model for gel collapse has been difficult due to the large number of parameters controlling the settling behaviour. In his $\mathrm{PhD}$ thesis Meeker [16] investigated the effect on delay time of a range of different sample parameters. The system under study was a colloid-polymer mixture (PMMA-polystyrene) of size ratio $\xi \approx 0.06$ $\left(a=300 \mathrm{~nm}, r_{g}=19 \mathrm{~nm}\right.$ at $\left.\mathrm{T}=19 \pm 2^{\circ} \mathrm{C}\right)$.

\section{Sample Composition}

The delay time, $\tau$, at moderate volume fractions, $\phi=0.20 \rightarrow 0.30$, has an exponentiallike dependence on free volume polymer concentration $C_{p}^{f r e e}$. This dependency is more pronounced at higher volume fraction. At lower volume fraction the dependency of $\tau$ on $C_{p}^{\text {free }}$ is linear. 
If the collapse of the gel network is driven by thermal rearrangements then we may expect the time scale of the restructuring, and so the delay time, to be related to the Boltzmann distribution, i.e. $\sim \exp \left(-U_{\text {dep }} / k_{B} T\right)$. We recall from equation 2.7 that the depletion strength $U_{d} e p$ is proportional to the free volume polymer concentration $C_{p}^{\text {free }}$ therefore it is not unrealistic to expect an exponential dependency of delay time on polymer concentration.

At stronger interaction potentials we might expect gel collapse to be driven by weight induced rearrangements. In this case we expect the rigidity of the gel network to increase with the bond strength between particles. This will increase the delay time but how it will depend on $C_{p}^{\text {free }}$ is not obvious.

The strong dependency of $\tau$ on $\phi$ can be seen by comparing the delay time of gels with the same concentration of polymer but different volume fractions. At $C_{p}^{\text {free }} \approx 6.00$ $\mathrm{mgcm}^{-3} \tau(\phi=0.20) \approx 1$ hour, $\tau(\phi=0.30) \approx 4$ hours and, $\tau(\phi=0.40) \approx 50$ hours. This strong dependency is due to the reduction of free volume as $\phi$ increases, recall equations 2.5 and 2.6. This has the effect of enhancing $C_{p}$, equation 2.8 , and thus the strength of the depletion interaction.

\section{Sample Size: Strong and Weak Behaviour}

According to Meeker [16], the transient gels can be divided into two categories based on the response of the delay time to varying the initial suspension height. At high polymer concentrations the delay time increases as the initial height of the gel decreases. These gels are known as strong as they are found at higher polymer content and therefore stronger interaction potential. At lower polymer concentrations the delay time is independent of the initial height. These gels are known as weak.

The crossover from weak to strong behaviour was explained by Meeker [16] as a consequence of a change in collapse mechanism. At low polymer concentrations the collapse of a gel is envisaged to be driven by intrinsic rearrangement such as thermally induced bond breaking leading to depercolation of the gel network. Such processes are height independent. At higher polymer concentrations the collapse is driven by weight-induced rearrangements. The gel breaks apart under its own weight which then causes back flow of solvent leading to the destruction of the gel. These processes should show some height dependency. 
Width dependency has also been found in other systems exhibiting delayed sedimentation. It has been found that gelled suspensions of needle-like iron oxide particles show width dependent delay times at high enough concentrations [24]. In this case the delay time increases when the diameter of the cylindrical cell is decreased. Similar behaviour has also been observed in the PMMA-polystyrene system [25].

It has been suggested by Meeker [16] that strong gels may show weak, i.e. heightindependent, behaviour if they could be made tall enough. We shall return to this idea in chapter 6 where we present the results of a more detailed study of size dependency in colloid-polymer gels.

\section{Sample Geometry}

The shape of the sample cell can also affect the measured delay time of a gel but in a very confusing way. It has been found [16] that delay time of a weak gel ( $\tau \approx 5$ hours) in a $1 \mathrm{~cm}$ circular cross-section cell is consistently half that of the same gel in a $1 \mathrm{~cm}$ square cross-section cell. Why this should be is not clear especially when we consider the height and width independent nature of the delay time in weak gels.

The delay time of a strong gel $(\tau \approx 20$ hours) remains unchanged in making the same cell change. However, by increasing the diameter of the cell to $1.5 \mathrm{~cm}$ the delay time falls dramatically to 5 hours. Increasing the sample width further to $3 \mathrm{~cm}$ has no effect on the delay time.

All of these experiments were carried out at constant sample height of $2 \mathrm{~cm}$.

\subsubsection{Imaging of Gel Collapse}

Some imaging of the outer surfaces during gel collapse has shown some interesting features. In his thesis, Meeker observed the top half of a weak gel sample ( $\tau \approx 3.5$ hours) in a plane adjacent to the cell wall [16]. Good contrast images were obtained by illuminating a region of the suspension with a thin sheet of white light in a plane perpendicular to the viewing direction. Here we present a summary of his findings. 
Initially the settling of the suspension was confined to the top corners of the sample. This was an effect of meniscus draining and the bulk of the sample remained unaffected. As the suspension slowly settled the surface began to buckle. The top of the suspension appeared bright indicating that it may have been more dense. As the end of the delay period approached, vertically oriented channels began to move upwards in the sample. Colloidal material carried by solvent flow was seen moving up through these channels. The top corners began to show bright regions of a coarse structure indicative of large density fluctuations. These regions sank slowly into the suspension at first increasing the amount of surface buckling. The coarse regions then suddenly began to settle much faster breaking up the structure of the whole sample as they propagated. The gel was now in the rapid collapse stage. The suspension surface was now flat and the whole sample has the appearance of the coarse bright material. Material could be seen circulating in the settling suspension. From the bottom a structureless region could be seen growing upwards indicating the growth of a dense amorphous sediment.

In his study, Meeker did not image the collapse of a strong gel. If the occurrence of strong gel behaviour is due to a change from intrinsically led collapse to weight-induced collapse then perhaps there may be an observable difference in the settling processes of strong and weak gels. This matter will be addressed later on in chapter 4 where we present new dark-field observations on a series of gels with a wide range of delay times.

\subsubsection{Low Shear Rheology of Transient Gels}

One explanation for the delayed settling behaviour of transient gels may be the presence of a significant yield stress. However, a low shear rheological study of colloid-polymer gels [16] finds that no yield stress exists within the limits of experiment. An upper bound of $10^{-3} \mathrm{Nm}^{-2}$ has been placed on the yield stress. It should be noted however that this is a measure of the yield stress due to shearing which may not be as important as a compressional yield-stress in understanding gel collapse.

The viscosity of a transient gel at low shear rates decreases rapidly as applied shear stress increases. It has been suggested that this strong shear-thinning property of transient gels can lead to the yield-like settling behaviour [16]. 
The delay time of a gel decreases as the applied shear stress increases for all but the lowest applied stresses. Increasing the applied shear stress also increases the rapid settling velocity and the volume fraction of the final sediment formed after collapse. This can be explained by the fact that only the most compact clusters will survive as the applied stress is increased. The most compact clusters will be the ones which settle fastest and pack the most efficiently in the final sediment.

At low constant shear stresses, the viscosity of a transient gel increases to a maximum value and then sharply decreases. Comparisons with the settling profile measured at the same time show that this apparent strengthening of the gel occurs during the delay time. The sudden drop in viscosity occurs around the same time as the gel begins to settle rapidly. 


\section{Chapter 3}

\section{Experimental Methods}

\subsection{Introduction}

In this chapter we introduce the two main experimental techniques used to study colloidpolymer gels in this work: dark-field imaging and ultrasonic concentration profiling.

\subsection{Dark-Field Imaging}

One of the great advantages in studying colloidal systems is the accessibility of the length scale of colloidal structures to visual observation ( $\sim$ millimetres in colloidpolymer gels). These macroscopic sizes allow us to probe colloidal structures using radiation in the visible light spectrum and one obvious way to study the collapse of colloid-polymer gels is to observe them directly using video recording techniques.

However, for the system studied here there exists a considerable problem as the contrast between different regions in the bulk of the suspension is very poor. This occurs because high contrast imaging requires a large difference between the refractive indices of the particles and the suspending solvent. Unfortunately, this situations leads to a large amount of multiple scattering by the sample which makes imaging the bulk impossible. We note that previous observations of structure formation in systems showing delayed sedimentation have been made only on the surfaces of the suspension [16, 24]. 
To reduce the amount of multiple scattering we combine two solvents (tetralin and cisdecalin) to match the refractive of the solvent mixture to that of the particles. However, refractive-index-matching leads to very poor contrast between different regions within the suspension under ordinary bright-field imaging conditions. To obtain good video pictures it is necessary to enhance the contrast in some way. The solution to the contrast problem employed in this work was to use dark-field imaging.

\subsubsection{Theoretical Background}

Figure 3.1 below shows the principle of dark-field imaging. Light is collimated by a lens and illuminates an object. In a nearly index-matched sample most of the light passes through undeviated. This makes up an unscattered beam and is represented by the solid rays in figure 3.1. Small refractive index inhomogeneities within the object will weakly scatter the incident light, dotted rays in figure 3.1. Scattered light is collimated by the objective and is finally focused to points in the image plane by the focusing lens. The unscattered light is focused to a point in the back focal plane of the objective lens and is then collimated by the focusing lens to illuminate uniformly the field of view in the image plane.

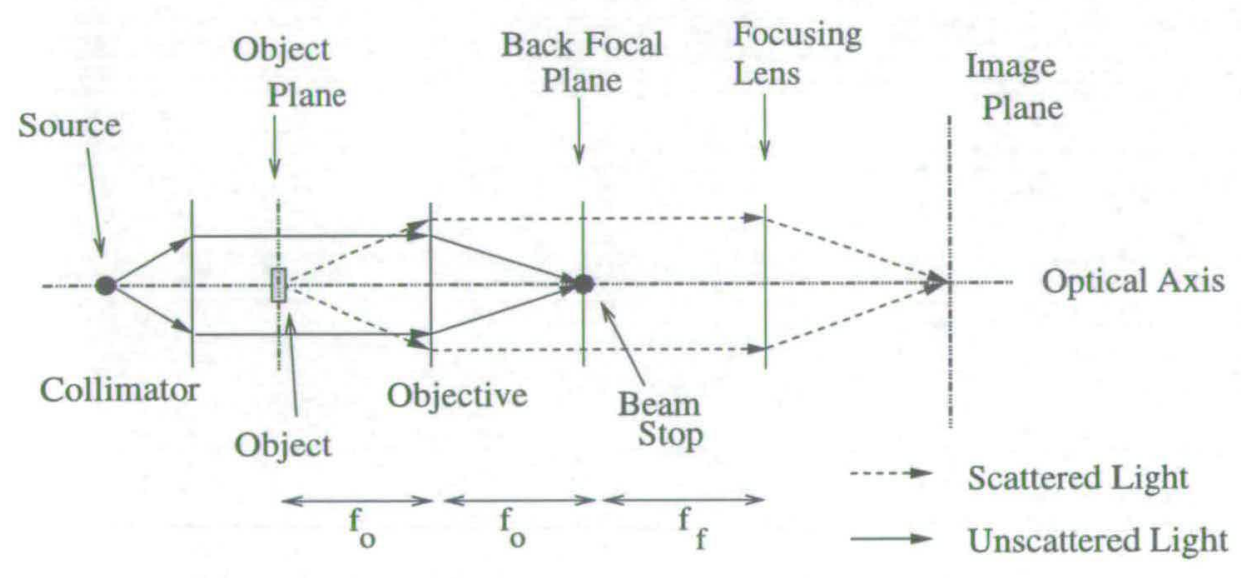

Figure 3.1: Schematic diagram showing the principle of dark-field imaging.

If both unscattered and scattered light is allowed to reach the image plane then the image formed is a combination of them both, giving a conventional bright-field image. For samples in which scattering is weak, i.e. small refractive index inhomogeneities, we observe very little detail using bright-field imaging. 
If we in some way stop the direct beam from reaching the imaging plane then only light scattered by the sample will be collected. This is how a dark-field image is produced. The name refers to what is observed when no sample is present in the object plane, i.e. a dark field of view. There is more than one way to achieve dark-field imaging [26]. In this work we take advantage of the spatial separation of the unscattered and scattered light in the back focal plane of the objective lens. In this plane the unscattered light is focused to a point while the scattered light is distributed across the whole plane. By placing a small beam stop in this plane it is possible to block the unscattered while still allowing most of the scattered radiation to pass through as shown in figure 3.1.

\section{Depth of Field: The Strehl Limit for Defocus}

The depth of field for an optical system is the distance along the optic axis over which the image produced can be considered to be in focus. This is shown schematically in figure 3.2. An object in plane $P$ is imaged by a thin lens $L$ a distance $z$ downstream. The image is formed in the plane $P^{\prime}$ which is some distance $z^{\prime}$ downstream of the lens.

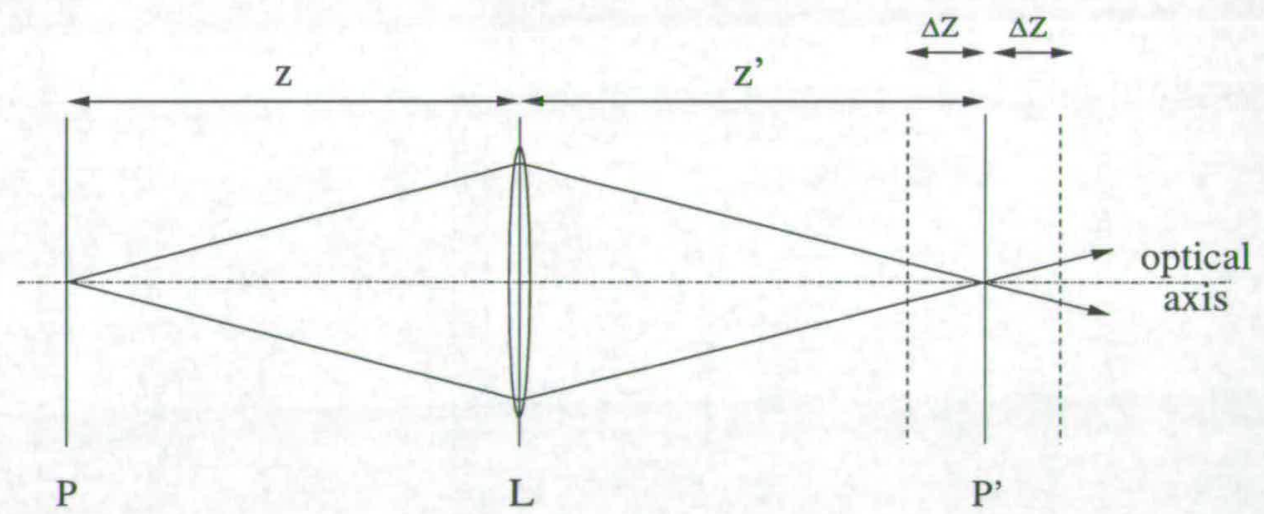

Figure 3.2: Schematic ray diagram of a single lens system. A focused image of an object in the plane $P$ will be formed in the plane $P^{\prime}$ if $1 / z+1 / z^{\prime}=1 / f$ where $f$ is the focal length of the lens $L$.

Moving the imaging plane by some distance $\Delta z$ causes the image to become defocused. However, small differences in the image plane's position will not have a significant effect on the quality of the image, i.e. it can still be regarded as in focus.

The limit at which defocus becomes significant is known as the Strehl limit [27] which 
is defined as the point where the central peak of the point spread function* has fallen to $80 \%$ of its ideal intensity. This occurs when the phase difference caused by defocusing reaches $\Delta \phi \sim \pi / 4$.

For the case where the object is at infinity, i.e the rays from the object are parallel when they arrive at the imaging lens, the Strehl limit for defocus is given by;

$$
\Delta z \leq \frac{1}{2}\left(\frac{f}{a}\right)^{2} \lambda
$$

where $\Delta z$ is half the depth of field, $f$ is the focal length of the lens, $a$ is the radius of the lens aperture and $\lambda$ is the wavelength of the incident illumination. Applying this equation to a typical objective lens used in the experiments $(a=20 \mathrm{~mm}, f=400 \mathrm{~mm}$, $\lambda=550 \mathrm{~nm}$ ) we estimate the theoretical depth of focus to be $2 \Delta z \leq 0.22 \mathrm{~mm}$.

The depth of focus of the system was measured experimentally by imaging a microscope graticule inclined at a known angle, $\theta$, to the beam direction at high magnification, see figure 3.3 .

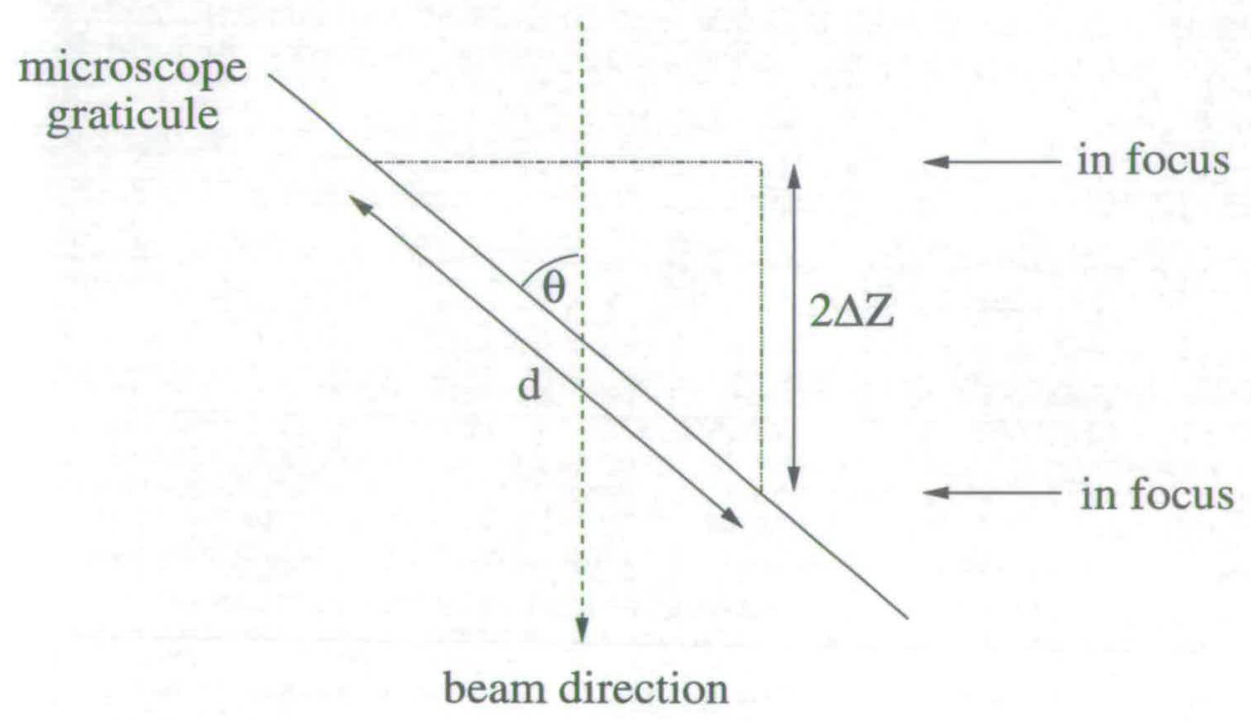

Figure 3.3: Diagram showing the orientation of a microscope graticule with respect to beam direction in order to measure the depth of field of the dark-field observation rig

*The point spread function is the function that describes the intensity distribution in the image plane 
From the above figure we see, by geometrical arguments, that the depth of field $2 \Delta z$ is given by

$$
2 \Delta z=d \cos \theta
$$

where $2 \Delta z$ is the depth of focus and $d$ is the lateral distance over which the graticule scale is in focus. Using this technique the depth of focus was found to be $0.4 \pm 0.1$ $\mathrm{mm}$ which is twice the theoretically expected value. To check this value, the graticule was fixed at right angle to the beam and imaged at high magnification. The graticule position was then varied in the direction of the beam and its displacement measured by a micrometer accurate to $0.01 \mathrm{~mm}$. Using this method the depth of focus was found to be $0.35 \pm 0.01 \mathrm{~mm}$.

\subsubsection{Experimental Setup}

Figure 3.4 shows a schematic diagram of the Dark-field Observation Rig (DOR). Samples are illuminated by a white light source (tungsten-halogen). A ground-glass screen is used to remove detail of the lamp filament from the final image. The light is then focused onto a small aperture of diameter 1-2 $\mathrm{mm}$ by the condenser lens. This small spot of light is then used as the illumination source.

By using a small aperture we produce a partially coherent source. This means that the images obtained by this method show some speckle pattern due to short range correlations in the illumination. This is especially obvious in regions of gel break up when scatterers are moving rapidly ${ }^{\dagger}$. Images are captured by a CCD camera and recorded on time-lapsed video. It is also possible to capture images onto computer disk by using image-grabbing software [28].

The lenses used in the DOR are achromatic doublets which have an anti-reflection coating. The objective lenses used have a useful aperture of $35 \mathrm{~mm}$ and nominal focal lengths of $400 \mathrm{~mm}$ or $200 \mathrm{~mm}$. The lenses used to form the final image on the CCD array have a useful aperture of $20 \mathrm{~mm}$ and nominal focal lengths of $50 \mathrm{~mm}$ or $125 \mathrm{~mm}$.

\footnotetext{
${ }^{t}$ This texture should not be linked to the presence of colloidal crystals in the sediment which have not been observed in these system as will be shown later
} 


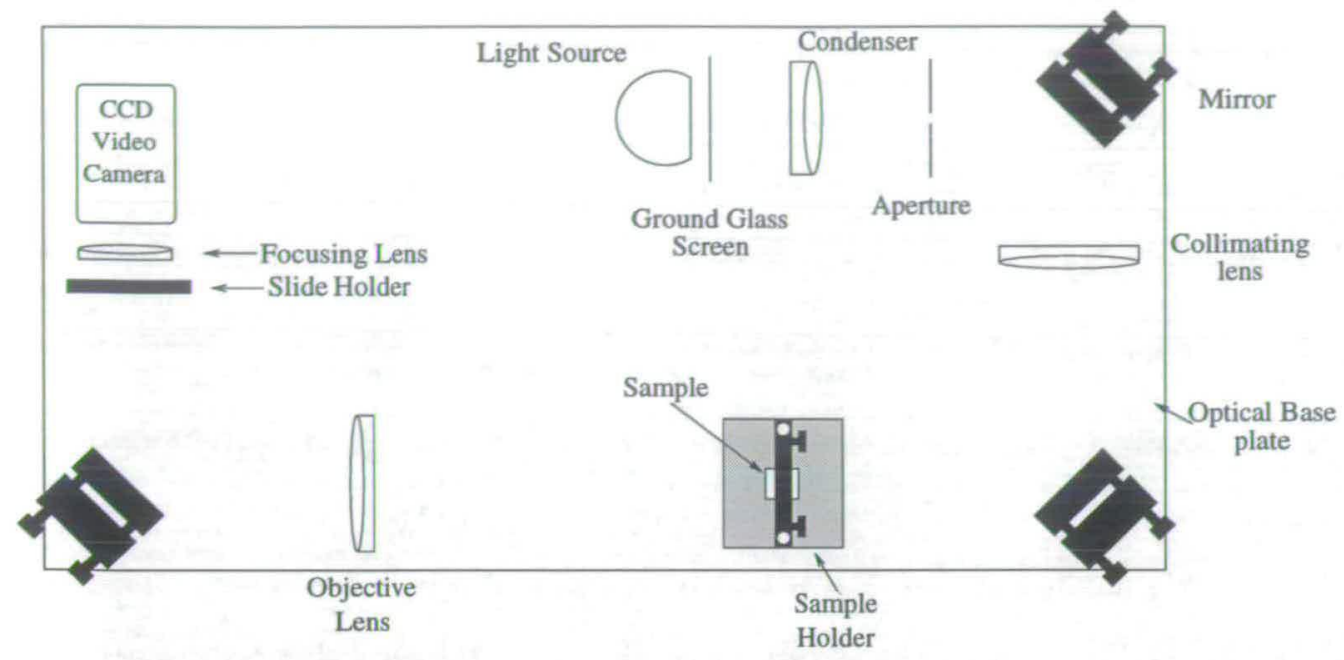

Figure 3.4: Schematic diagram showing the layout of the Dark-field Observation Rig (DOR). The temperature control apparatus is not shown.

The optical magnification of this system is given by the ratio of the focal lengths of the objective $\left(f_{o}\right)$ and imaging lenses $\left(f_{f}\right)$;

$$
M=+\left(\frac{f_{f}}{f_{o}}\right)
$$

In order to view the whole of a sample, typically $2 \mathrm{~cm}$ high and $1 \mathrm{~cm}$ wide, it is necessary to reduce the image size to fit onto the CCD array of the camera, i.e. have magnifications of less than one. The most commonly used optical set up comprises the $400 \mathrm{~mm}$ objective lens and the $50 \mathrm{~mm}$ imaging lens giving a magnification factor of $\mathrm{M}=50 / 400=0.125$. Thus, this optical setup is not a microscope but a macroscope [29].

The sample holder and the CCD camera are mounted such that they can be moved parallel to the beam direction and in the two perpendicular directions. The sample holder is enclosed in a temperature-controlled chamber, not shown in the above figure. This comprises four perspex boxes which fit together under an insulated lid to surround the sample holder in a sealed chamber. The temperature in the chamber is kept constant by pumping water through the boxes at a constant temperature. The two boxes perpendicular to the beam direction have a sealed region in which no water can enter. These windows are to ensure that the illuminating beam does not pass through any of the water which may affect the quality of the image. At typical operating temperatures, between $20^{\circ} \mathrm{C}$ and $25^{\circ} \mathrm{C}$ the chamber temperature varies by less than $0.5^{\circ} \mathrm{C}$. 


\subsubsection{Image Interpretation}

Under dark field imaging conditions, quiescent gel appears dark while gel which has begun to break up appears bright. This can be understood in terms of the small angle light scattering behaviour of gels [15]. At low angles the onset of gelation is observed by the presence of a ring of scattered light in Fourier space. During the quiescent stage this ring is static at $Q a \sim 10^{-1}$, where $a$ is the radius of the colloidal particles and $Q$ is the scattering vector. We also observe very slow speckle dynamics, i.e. to the eye the speckle pattern produced in the scattered intensity appears frozen in time. The onset of rapid collapse coincides with the collapse of the scattering ring to much lower angles $\left(Q a \sim 10^{-2}\right)$.

In our dark field observation set up the aperture of the objective lens has been chosen to be slightly smaller than the diameter of the small angle scattering ring of the quiescent gel. Thus, no scattered light is collected from a quiescent gel and so it will appear dark. As the gel collapses, the small angle ring shrinks in diameter and enters the aperture of the lens. Thus the light scattered by broken gel structures is collected. We therefore expect regions of broken gel to appear as bright areas in the image. Solvent and polymer molecules are not expected to be visible using this technique as they scatter light very weakly and far outside the aperture of the objective due to their small size.

Figure 3.5 shows the scattering geometry of the direct observation setup used in this study. The objective lens is placed at a distance equal to its focal length $f$ downstream of the sample. The lens has finite aperture of radius $r$. Only light which is scattered at an angle less than $\theta$ from the optic axis may enter the aperture of the lens and be detected.

For a typical objective lens $(f=400 \mathrm{~mm}, r=17.5 \mathrm{~mm})$ this angle is

$$
\theta=\tan ^{-1}\left(\frac{r}{f}\right)=\tan ^{-1}\left(\frac{17.5}{400}\right)=2.5^{\circ}
$$

This maximum angle corresponds to a scattering vector $\vec{Q}$ whose magnitude is 


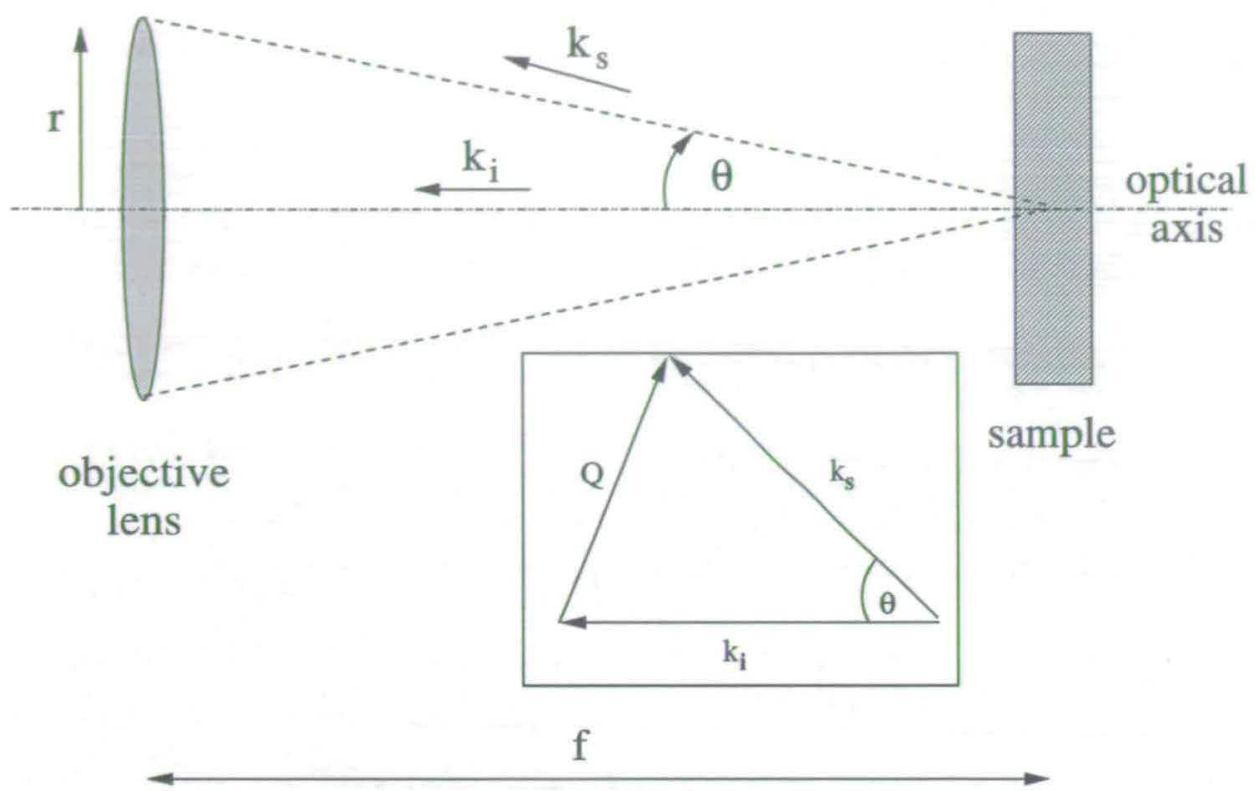

Figure 3.5: Schematic diagram showing the geometrical relation between the objective lens and the scattering source in the sample. Only light scattered at angles less than $\theta$ from the optic axis will enter the aperture of the lens, radius $r$, and be imaged. $f$ is the focal length of the objective. The directions of the incident and scattered propagating vectors $\overrightarrow{k_{i}}$ and $\overrightarrow{k_{s}}$ are indicated. Inset: The scattering vector $\vec{Q}$ is the difference between the vectors $\overrightarrow{k_{i}}$ and $\overrightarrow{k_{s}}$.

$$
Q=\frac{4 \pi}{\lambda} \sin \left(\frac{\theta}{2}\right)=\left(\frac{4 \pi}{5.5 \times 10^{-7}}\right) \sin \left(1.25^{\circ}\right)=5 \times 10^{5} \mathrm{~m}^{-1},
$$

where we have assumed a mean wavelength of $\lambda=550 \mathrm{~nm}$ for the white light source used. The mean radius of the colloidal particles used in this study was typically $a \approx$ $200 \mathrm{~nm}$ giving $Q a \approx 0.1$ for this particular scattering geometry.

\subsection{Ultrasonic Time-of-Flight Measurements}

We have also studied the time dependent concentration with height of a collapsing transient gel using ultrasound. Many methods for measuring the concentration of a settling suspension of particles are intrusive and disrupt the process under observation, e.g. sampling [30]. One non-intrusive method is to measure the speed of a pulse of ultrasound travelling through the suspension and to relate the pulse velocity to concentration. This section introduces some basic background to the field of ultrasonic profiling and describes the technique. 


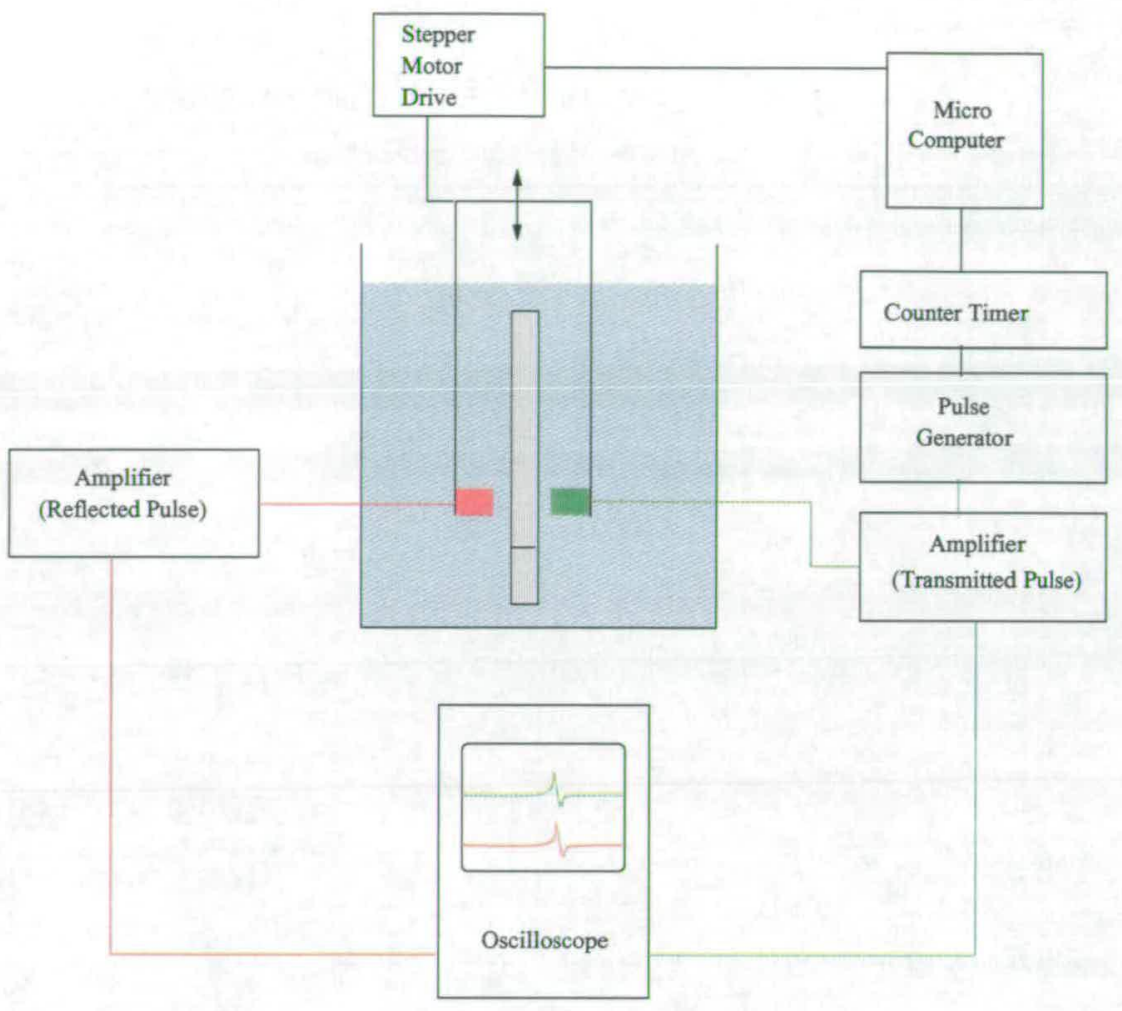

Figure 3.6: Schematic diagram showing the set up of the Ultrasonic rig at IFR

\subsubsection{Measuring Time-of-Flight}

Figure 3.6 shows a schematic representation of the ultrasonic rig at the Institute of Food Research (IFR) [31]. The sample cell is held on a rotating carousel which is enclosed in a water bath, the temperature of which is controlled to within $0.02^{\circ} \mathrm{C}$. This is necessary as the speed of ultrasound in many substances is highly sensitive to temperature variations. For example, the speed of sound in distilled water at atmospheric pressure increases steeply with increasing temperature around $T=20^{\circ} \mathrm{C}$, see figure 3.7 .

The water in the temperature bath also acts as an ultrasonic buffer between the transducers and the sample cell to reduce any loses due to mismatching of acoustic impedance of the two media. All materials have a characteristic acoustic impedance $Z=\rho c$ [33] where $\rho$ is the density of the material and $c$ is the speed of sound. Consider a sound wave of amplitude $A_{i}$ travelling from one medium of characteristic impedance $Z_{1}$ into another of impedance $Z_{2}$. Part of the initial wave will be reflected from the boundary between the two media and part will be transmitted. 


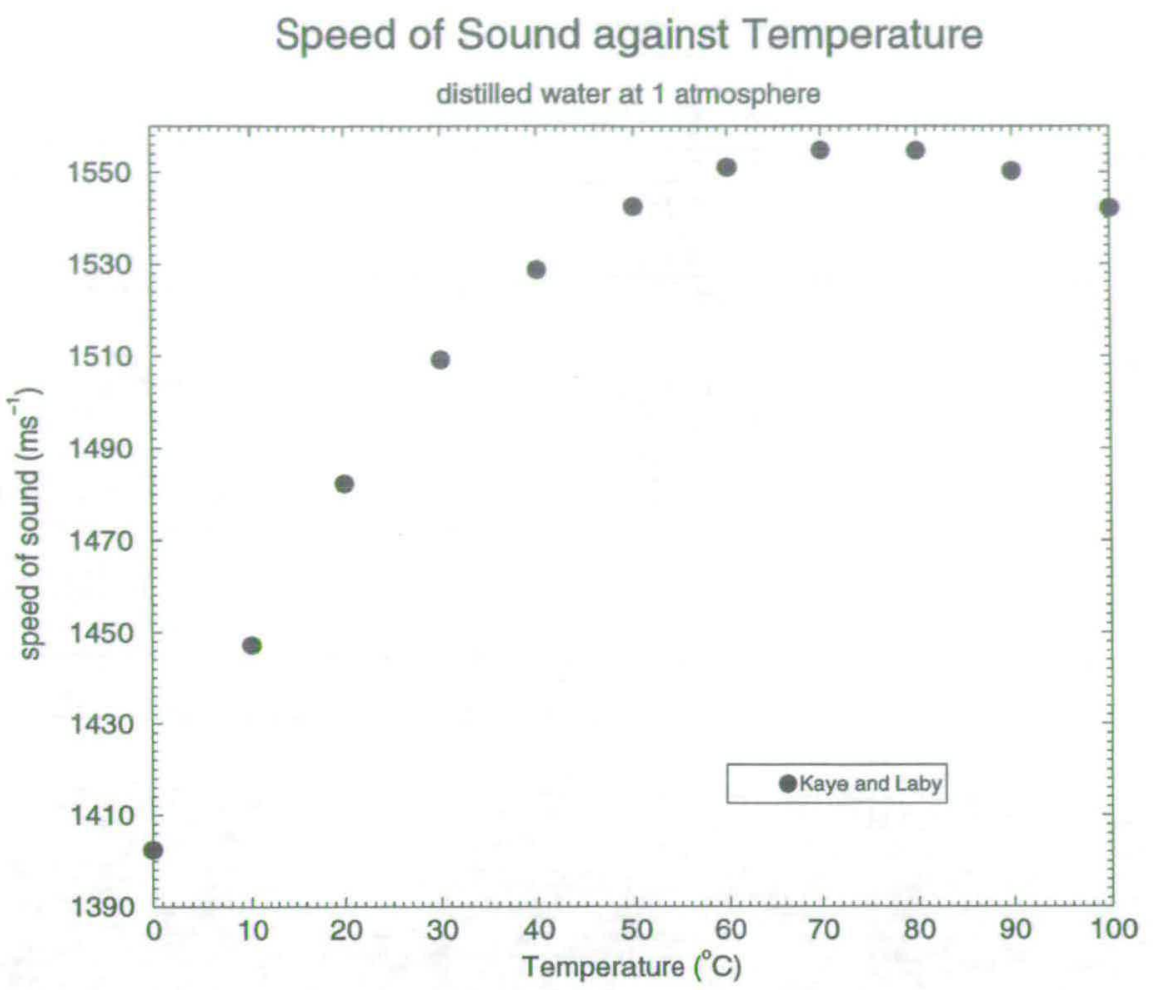

Figure 3.7: Graph of speed of sound against temperature for distilled water at 1 atmosphere. Data reproduced from Kaye and Laby [32].

The proportion of the initial wave which will be reflected can be written in terms of the impedance difference between the two media as [33]

$$
a_{r}=\left(\frac{Z_{2}-Z_{1}}{Z_{2}+Z_{1}}\right)^{2}
$$

where $a_{r}$ is known as the coefficient of reflectance. Any portion of the incident wave which is reflected from the boundary results in a loss of transmitted signal. Perfect transmission of the acoustic wave occurs when $a_{r}=0$, i.e. the characteristic impedances of the two media are the same. In practice this is not possible. However, we can increase the amount of signal transmitted by reducing the impedance difference as much as possible.

As an example of impedance matching we calculate $a_{r}$ for an air-perspex interface and a water-perspex ${ }^{\ddagger}$ interface. Typical characteristic impedances for air, water and perspex

\footnotetext{
${ }^{\ddagger}$ The sample cells used in this technique are constructed from perspex
} 
at $20^{\circ} \mathrm{C}$ are $Z($ air $)=430 \mathrm{kgm}^{-2} \mathrm{~s}^{-1}, Z$ (water $)=1.5 \times 10^{6} \mathrm{kgm}^{-2} \mathrm{~s}^{-1}$, and $Z$ (perspex) $=3.2 \times 10^{6} \mathrm{kgm}^{-2} \mathrm{~s}^{-1}[33]$. Thus,

$$
\begin{gathered}
a_{r}(\text { air - perspex })=\left(\frac{Z_{\text {perspex }}-Z_{\text {air }}}{Z_{\text {perspex }}+Z_{\text {air }}}\right)^{2}=\left(\frac{3.2 \times 10^{6}-430}{3.2 \times 10^{6}+430}\right)^{2}=1.00, \\
a_{r}(\text { water }- \text { perspex })=\left(\frac{Z_{\text {perspex }}-Z_{\text {water }}}{Z_{\text {perspex }}+Z_{\text {water }}}\right)^{2}=\left(\frac{3.2 \times 10^{6}-1.5 \times 10^{6}}{3.2 \times 10^{6}+1.5 \times 10^{6}}\right)^{2}=0.13
\end{gathered}
$$

From the above calculations it is clear that we will obtain good transmission of the ultrasonic signal by placing the sample in bath of water as opposed to conducting the experiment in air where the pulse will be reflected from the cell wall.

\section{The Sample Cell}

The cell used is rectangular in shape with dimensions $14 \mathrm{~mm}$ width by $10 \mathrm{~mm}$ depth by $192 \mathrm{~mm}$ height. It is made of Perspex (PMMA) so as to reduce any reflections of the ultrasonic pulse inside the cell walls caused by the difference in characteristic impedance of the cell material and the surrounding media. The result of the impedance mismatch is to produce extra reflected pulses in the received signal which, if large enough, can mask the true signal. Perspex cells are used as they have a characteristic impedance close to that of water and so are able to reduce the intensity of the reverberation signal.

The cell has two chambers which are separate from each other. The upper, larger chamber is where the sample is placed. The lower chamber, known as the false base, is filled with demineralised water and is used to make calibration measurements before each experimental run.

\section{The Ultrasonic Transducers}

The transducers are mounted opposite each other on a rigid carriage which may be moved vertically up and down the sample by a stepper motor. The minimum step size employed is $1 \mathrm{~mm}$. The transducers produce ultrasound with a typical centre frequency 
of $6.4 \mathrm{MHz}$, corresponding to a wavelength in water of around $250 \mu \mathrm{m}$ at $20^{\circ} \mathrm{C}$. The transducers measure $10 \mathrm{~mm}$ in diameter but produce an ultrasonic beam of estimated width $2 \mathrm{~mm}$ [34]. This reduction in beam width is due to two factors. Firstly only a small area in the centre of the transducers is ultrasonically active. Secondly, the pulse produced is strongly peaked around the centre of the transducer further reducing the width of the ultrasonic beam.

\section{Making the Measurement}

To create a pulsed signal for the ultrasonic transducers, a function generator is used to produce a half sine wave. This signal is sent to a pulse generator which produces pulses at a frequency that can be continuously varied. The pulse output by the generator is then passed to the transmitter transducer via a linear amplifier. The signal received once it has travelled through the sample is passed from the receiving transducer through another linear amplifier to a calibrated attenuator. The two pulses are then compared, by eye, on an oscilloscope.

To find the time-of-flight the time interval between the input pulses is varied manually using the pulse generator. When the interval between transmitted pulses matches the time-of-flight, the transmitted pulse will coincide on the oscilloscope screen with the received pulse from the previous transmission.

The instrument is controlled by a microcomputer which is interfaced with a countertimer and the stepper motor driving the transducer carriage and the sample carousel. When the two pulses are made to coincide as described then the microcomputer is allowed to log the time-of-flight, the height of the transducers and the absolute time. The computer takes an average of 500,000 time-of-flight measurements in order to achieve an accuracy of $1 \mathrm{~ns}$ in $25 \mu \mathrm{s}$.

\subsubsection{Calculating the Speed of Sound in the Sample}

By measuring the pulse time-of-flight between the transducers we can calculate the average velocity of pulse since the transducer separation is known. We wish to know the velocity of sound in the sample dispersion. However, it is not only the sample 
which contributes to the time-of-flight of the ultrasonic pulse as it travels between the transducers. The pulse also has to pass through the cell walls and the water in the temperature bath which will both contribute to the pulse time-of-flight. There are also time delays introduced by the various electronic devices used to modify and measure the pulse to be accounted for.

To find the time-of-flight due to the sample dispersion, and thus the speed of sound in the sample, we must subtract all the non-sample contributions to time-of-flight from the measured raw time-of-flight. This is achieved by measuring the time-of-flight in two reference fluids in which the velocity of sound is well characterised. Since we know the velocity of sound, and so the time-of-flight of a pulse of ultrasound, in these two liquids we can calculate the time delay introduced by all the other sources. The two liquids used are demineralised water and hexane. Once the delay constant is known it can be subtracted from all measurements made on a sample to give a true time-of-flight due to the dispersion.

Before any measurement of the time-of-flight are made in a sample we first make a measurement in the false base which is filled with demineralised water. The false base is a small chamber, about $5 \mathrm{~cm}$ high, which is separated from the main sample chamber. Its purpose is to allow further correction to the delay constant to be made to each measurement of the time-of-flight in any suspension. These arise from small changes which occur in the sample cell over time, e.g. the cells are known to absorb small amounts of water from the temperature bath which will affect the time-of-flight of the ultrasonic pulse.

\subsubsection{Calculating the Sample Concentration}

It is now possible to measure the time-of-flight and thus the velocity of ultrasound in a colloidal suspension. However, in order to calculate the volume fraction of colloid from the velocity profiles the relationship between these two quantities must be deduced.

In general it is a non-trivial exercise to find the relationship between dispersion concentration and the speed of sound. The speed at which the pulse propagates depends not only upon the concentration of particles but also upon their size, shape, excitation 
frequency and the physical properties of both the dispersed and continuous phases. However, when the particles are much smaller than the wavelength of ultrasound, and there is a large enough difference in the speed of sound between the materials which make up the dispersed and continuous phases, then the effect of particle concentration greatly outweighs any other contribution. Under these circumstances we can assume that the dispersion behaves like a simple mixture of its components. This approach leads to a quadratic relationship between pulse speed and volume fraction known as the Urick equation [35]. This can be written as

$$
\left(\frac{v}{v_{c}}\right)^{-2}=\left[1-\phi\left(1-\frac{\rho_{d}}{\rho_{c}}\right)\right]\left[1-\phi\left(1-\frac{\rho_{c} v_{c}^{2}}{\rho_{d} v_{d}^{2}}\right)\right],
$$

where $v$ is the speed of ultrasound through the dispersion, $v_{c}$ and $v_{d}$ are the speed of ultrasound through the continuous and dispersed phases respectively, and $\rho_{c}$ and $\rho_{d}$ are the respective densities.

By solving equation 3.9 for the dispersed phase volume fraction, $\phi$, we can calculate the concentration of a dispersion at a particular point if the speed of ultrasound at that point is known.

\section{Calibration}

To use the Urick equation (equ. 3.9 ) we need to know the values of $v_{c}, v_{d}, \rho_{c}$, and $\rho_{d}$ for our specific system. We find these values by measuring the velocity of ultrasound in samples of pure colloid of known volume fraction, see table 3.1. This produces some points on a calibration curve to which we fit a quadratic relationship in $\phi$ where $v_{c}, v_{d}, \rho_{c}$, and $\rho_{d}$ are variables which are allowed to vary in order to find the best fit. For the system used in these experiments the best values to be input into the Urick equation were found to be $v_{c}=1433.9 \mathrm{~ms}^{-1}, v_{d}=1991.0 \mathrm{~ms}^{-1}, \rho_{c}=892.64 \mathrm{kgm}^{-3}, \rho_{d}=$ $1143.78 \mathrm{kgm}^{-3}$.

The continuous phase in colloid-polymer gels will not only comprise solvent but will also contain some polymer. To account for this we also measure the velocity of ultrasound in the polymer stock, see table 3.1 . The speed of sound in the polymer stock is 


\begin{tabular}{|c|c|}
\hline$\phi$ PMMA & velocity $\left(\mathrm{ms}^{-1}\right)$ \\
\hline 0.4814 & 1593.65 \\
0.3755 & 1546.50 \\
0.2620 & 1506.10 \\
0.1695 & 1478.20 \\
0.0901 & 1456.00 \\
\hline pure cis-decalin & 1433.90 \\
Polymer Stock & 1435.35 \\
\hline
\end{tabular}

Table 3.1: Table of ultrasound velocity with different colloid volume fractions. The velocity of ultrasound is also shown for pure solvent and the polymer stock solution.

less than $2 \mathrm{~ms}^{-1}$ faster than that in pure cis-decalin. This leads to a less than $1 \%$ error in determining the volume fraction from velocity data. However, in producing concentration data we have made small alterations to $v_{c}$ and $\rho_{c}$ to account for the different amounts of polymer present in the continuous phase.

\subsubsection{Multiple Scattering}

It is known that the effects of multiple scattering in many colloidal suspensions can be quite significant [36]. However, the Urick equation takes no account of multiple scattering from the dispersion. By using measured values for the variables in the Urick equation we are able to compensate for small scattering effects when calculating the volume fraction. However, this calibration has been performed on non-aggregated colloidal suspensions whilst we are interested in measuring the concentration of an aggregated structure. The effects of multiple scattering are dependent upon the structure of what is causing the scattering. It is known that aggregated dispersions scatter the ultrasonic pulse more than non-aggregated dispersions [37]. This will introduce further error into our time-of-flight measurement and so to the final volume fraction measurement. The magnitude of this error is not know but we shall assume that it is small enough to be unimportant. 


\section{Chapter 4}

\section{Direct Observation of Transient Gel Collapse}

\subsection{Introduction}

Although mesoscopic in size, colloids can produce macroscopic structures. This allows us to probe such structures with radiation in the visible spectrum. Thus one of the simplest and yet most powerful techniques we can use to study colloidal systems is by direct observation.

Preliminary observations of gel collapse in this colloid-polymer system have shown some interesting features during the delay and rapid collapse stages [16] (see also section 2.6.3 for a brief summary). However, only the top half of the sample could be viewed. Other studies [24] have only been able to image surface features.

The main advantage our imaging technique has over previous studies is the ability to image in the bulk of the suspension. This has been achieved by using a darkfield imaging technique combined with refractive-index-matching of the samples under study. The index-matching is important in reducing the effects of multiple scattering. However, this leads to poor contrast in the suspension and we use a dark-field technique where only light scattered by the sample is collected to enhance the contrast. The details of the observation technique employed are discussed in more detail in section 3.2 . 


\subsection{The Model System}

The colloidal particles used in this study are sterically-stabilised PMMA hard spheres dispersed in a mixture of tetralin (tetrahydronaptheline) and cis-decalin. A mixture of two solvents is used to produce a suspending medium with a refractive index approximately equal to that of the PMMA particles. This index matching of the solvent and particles is necessary to produce good imaging conditions as explained in section 3.2. The spheres have radius $a=186 \mathrm{~nm}$ and size-polydispersity $\sigma \approx 5 \% *$.

The non-adsorbing polymer added to the colloid is polystyrene (PS) which is also dissolved in the same solvent mixture. The polystyrene has a weight-averaged molecular mass of $M_{w}=320,000 \mathrm{gmol}^{-1}$. According to Berry [38] the radius of gyration $r_{g}$ of a polystyrene coil suspended in cis-decalin at a temperature $T$ (in kelvin) is given by

$$
r_{g}=r_{g}^{\theta} \sqrt{1+\frac{134}{105} z}
$$

where $r_{g}^{\theta}=0.0276 \sqrt{M_{w}}$ is the radius of gyration at the theta temperature $T_{\theta}\left(T_{\theta}=\right.$ $12.5^{\circ} \mathrm{C}$ for polystyrene in cis-decalin) and $\mathrm{z}$ is the Fixman parameter

$$
z=0.00975 \sqrt{M_{w}}\left[1-\left(\frac{T_{\theta}}{T}\right)\right] \quad .
$$

Following this approach we find a radius of gyration of $r_{g} \approx 17 \mathrm{~nm}$ for polystyrene in cis-decalin at $25^{\circ} \mathrm{C}$. This produces a polymer to colloid size ratio of $\xi \approx 0.09$.

The effect of adding tetralin to the solvent mixture is to swell the polymer coils as tetralin is a better solvent for polystyrene than cis-decalin. For such small polymers we assume that this swelling is relatively small and so we estimate that the size ratio of the system used here is $\xi \sim 0.1$. We therefore do not observe distinct liquid and gas phases as the range of the interaction is too short.

Although no extensive study of the phase diagram has been made in this particular work, no evidence for liquid and gas phases has been found indicating that the polymer has not swollen significantly in the tetralin cis-decalin mixture.

\footnotetext{
*Particle size and polydispersity were determined by A. Schofield from static light scattering measurements.
} 
The polydispersity in molecular mass of the polymer coils can be calculated from the ratio of the weight-averaged molecular mass to the number-averaged molecular mass $M_{w} / M_{n}$. This quantity is commonly quoted by polymer manufactures and is related to the polydispersity in $M_{w}$ by

$$
\sigma_{M_{w}}^{2}=\frac{M_{w}}{M_{n}}-1
$$

which gives a polydispersity in the ideal radius of gyration of $\sigma_{r_{g}^{\theta}}=\sigma_{M_{w}} / 2$ [7]. For the polymer used in this study we calculate a polydispersity in $r_{g}^{\theta}$ of $10 \%$ from the manufactures quoted value of $M_{w} / M_{n}=1.04$.

\subsection{Sample Preparation}

Firstly a batch of solvent mixture is made up with the required ratio (by volume) of tetralin to cis-decalin, $V_{t} /\left(V_{t}+V_{d}\right) \approx 0.35$, giving a solvent refractive index of 1.499 . This was found to be an ideal index-match between the particles and solvent for our dark-field imaging technique. The refractive index of the solvent stock is measured to an accuracy of \pm 0.002 using an Abbe refractometer on a regular basis to check for deviations caused by the tetralin and cis-decalin evaporating at different rates. Any small changes in refractive index are corrected by adding small amounts of either solvent to compensate (usually tetralin as this evaporates faster than cis-decalin).

The polymer stock is made by placing a known mass of solid polystyrene in a container and then adding a known amount of the solvent mixture by weight. This is then mixed by slow tumbling overnight to dissolve the polymer in the solvent completely. The polymer stock is usually made up to a concentration of $C_{p s} \approx 15 \mathrm{mgcm}^{-3}$.

A stock of PMMA colloidal particles is then prepared in the same solvent mixture. This is done by centrifuging a dispersion in cis-decalin until a compact sediment collects in the bottom of the sample bottle. The excess solvent is then removed and replaced by the index-matched solvent mixture. The particles are then re-dispersed in the new solvent mixture. This procedure, known as washing, is repeated until the refractive index of the solvent matches that of the solvent stock. 
The colloid stock is then calibrated by a similar method to washing. The stock sample is centrifuged until a compact sediment forms at the bottom of the bottle. The excess solvent is then removed and the sediment is weighed. The sediment is assumed to have a volume fraction of $\phi=0.65$ [39]. A known volume of the solvent mixture is then added to the compact sediment to produce a colloid stock solution with volume fraction $\phi \approx 0.50 \pm 5 \%$.

Within the coexistence region $\left(\phi_{F}=0.494 \leq \phi \leq \phi_{M}=0.545\right)$ the fraction of crystalline phase present increases linearly with the volume fraction, see figure 4.1 . This property of hard-sphere colloids can be used to calibrate the colloid stock to a higher accuracy than the centrifuging method previously described. However, high accuracy in determining the composition of samples is not required in this study as we are interested in comparing a sequence of samples with the same volume fraction but different polymer contents, i.e. different delay times.

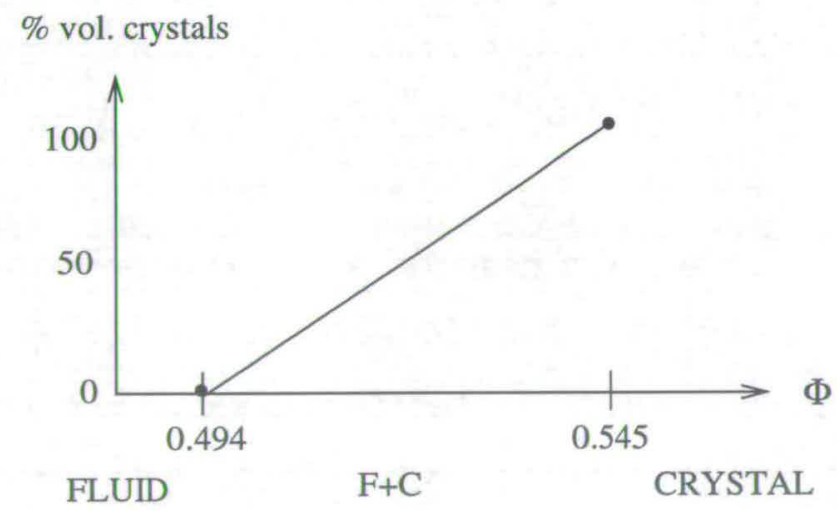

Figure 4.1: Schematic diagram showing the linear relationship between colloid volume fraction and percentage quantity of crystal phase within the fluid-crystal coexistence region of the hard-sphere phase diagram.

Measured amounts of each component (colloid and polymer stocks and solvent mixture) are then added by weight to a glass sample cell and mixed well to produce a sample of the desired colloid volume fraction $\phi$ and polymer concentration $C_{p}\left(\right.$ in $\left.\mathrm{mgcm}^{-3}\right)$.

\subsubsection{The Sample Cell}

The sample cells used for observations are of square cross-section $(1 \mathrm{~cm} \times 1 \mathrm{~cm})$ and are typically $4 \mathrm{~cm}$ in height. They are sealed with a teflon stopper which is fitted with a 
viton O-ring in order to reduce solvent evaporation. The sample cell is typically halffilled in order to make a standard sample of around $2 \mathrm{~cm}$ height. The cells are made of glass with four clear windows on the long dimension which are flat to within a micron. This makes them good cells for dark-field observations.

\subsubsection{Errors}

Uncertainties in the final sample composition $\left(\phi, C_{p}\right)$ arise from (i) uncertainties in the concentration of solvent mixture, and polymer and colloid stock solutions and (ii) solvent evaporation from the sample once it has been placed in the sample cell.

\section{Solvent Stock}

Uncertainty in the composition of the solvent stock arises due to errors in measuring the masses of the two solvents combined to form the index-matched mixture. Using the literature values for the solvent densities [40] we estimate the error on the density of the two solvents to be $\Delta \rho_{d}=\Delta \rho_{t}= \pm 0.00005 \mathrm{gcm}^{-3}$ which is a relative error of $\Delta \rho_{d}=\Delta \rho_{t}= \pm 0.005 \%$.

The error in measuring the masses of solvents mixed to form the mixture is $\Delta m_{d}=$ $\Delta m_{t}= \pm 0.001 \mathrm{~g}$. Typical masses measured are of the order of $10 \mathrm{~g}$ leading to a relative error on the mass of the two solvents measured of $\pm 0.01 \%$. This leads to a relative error in the density of the solvent mixture of $\Delta \rho_{s}=0.04 \%$.

Upon combining the errors involved in producing the solvent mixture we calculate the relative error on the mixing ratio $\left(V_{t} / V_{t}+V_{d}\right)$ to be around $\pm 0.03 \%$.

\section{Colloid Stock}

By comparison with colloid stock calibrated by the crystallisation method, the volume fraction of the colloid stock obtained by centrifugation is on average $5 \%$ higher than expected for particles of approximately $200 \mathrm{~nm}$ radius [41]. Even a small size polydispersity leads to a much more efficiently packed sediment than is predicted by random 
close packing [39]. Therefore the sediment produced by centrifuging has a higher volume fraction than the assumed value of $\phi=0.65$. By using the centrifugation method to calibrate the colloid stock a systematic error of $5 \%$ has been introduced into the calculated volume fraction.

Further error in the colloid stock volume fraction $\phi_{c s}$ is introduced by uncertainties in the balance used to measure the mass of solvent added to the dense colloidal sediment. It is estimated that all masses on the balance are accurate to $\pm 0.001 \mathrm{~g}$ which leads to a further uncertainty on $\phi_{c s}$ of $\pm 0.01 \%$.

\section{Polymer Stock}

Uncertainty in the composition of the polymer stock solution can arise from errors in the solvent stock solution and from measurement errors in weighing the dry polystyrene.

Making up the polymer stock requires us to measure out a small quantity of dry polystyrene $(\sim 0.1 \mathrm{~g})$ in a heavy container $(\sim 20 \mathrm{~g})$ and add to it a large mass of solvent stock $(\sim 10 \mathrm{~g})$. The relative error in measuring the mass of the dry polystyrene is calculated at $\Delta m_{p}= \pm 1 \%$ which is significantly larger than the error on the mass of solvent stock weighed, $\Delta m_{s}= \pm 0.01 \%$.

The concentration of polymer in the stock solution is given by

$$
C_{p s}=\frac{m_{p}}{V_{p}+V_{s}}=\frac{m_{p}}{\frac{m_{p}}{\rho_{p}}+\frac{m_{s}}{\rho_{s}}}
$$

Where $m_{p}$ is the mass of polymer measured out, $V_{p}$ and $V_{s}$ are the volume of polymer coils and solvent in the stock solution, $\rho_{p}$ and $\rho_{s}$ are the densities of the polymer and the solvent mixture.

The relative errors on the mass and density of the solvent mixture as well as in the density of the polymer are very small in comparison with $\Delta m_{p}\left(\Delta \rho_{s}= \pm 0.04 \%, \Delta m_{s}=\right.$ $\pm 0.01 \%$, and $\left.\Delta \rho_{p}= \pm 5 \times 10^{-3} \%\right)$. The uncertainty in the polymer concentration is then due only to the uncertainties in the mass of polymer $m_{p}$ and the volume of the polymer coils $V_{p}$. The overall error in the concentration of the polymer stock is thus calculated to be $\Delta C_{p s}= \pm 2 \%$. 


\section{Sample Composition}

To produce a sample of desired volume fraction and polymer concentration, known quantities of the three stock solutions (solvent, colloid, and polymer) are mixed in the sample cell. The volume fraction $\phi$ and the polymer concentration $C_{p}$ in the sample can be calculated from

$$
\phi=\frac{V_{c s}}{V_{c s}+V_{p s}+V_{s s}} \phi_{c s} \quad C_{p}=\frac{V_{c s}}{V_{c s}+V_{p s}+V_{s s}} C_{p s}
$$

where $V_{c s}, V_{p s}$, and $V_{s s}$ are the volumes of colloid, polymer and solvent stock solutions added to the sample. $\phi_{c s}$ and $C_{p s}$ are the volume fraction of the colloid stock and the polymer concentration of the polymer stock.

The relative error in the sample composition due to uncertainties in the concentrations of the solvent mixture, and polymer and colloid stock has been calculated to be $\Delta \phi=$ $\pm 1 \%$ and $\Delta C_{p}= \pm 2 \%$.

\section{Evaporation}

Evaporation of solvent has in all but a few exceptional cases no significant effect on sample composition. Once in the sample cell, evaporation is negligible which is checked regularly by weighing the sample. Evaporation of the stock solutions is more significant due to poorer seals on the stock bottles. As already mentioned the solvent stock is checked regularly by measuring its refractive index. Any variation in the refractive index of the solvent mixture, and thus its composition, is corrected by adding small quantities of either tetralin or cis-decalin.

\subsection{The Sedimentation Profile}

The plot of suspension height against time known as the sedimentation profile is a useful quantitative measure from which we can extract much information about the settling process. As already mentioned in section 2.6.1 the sedimentation profile for a suspension undergoing delayed sedimentation has three characteristic stages. An 
initial delay period during which little or no settling is observed is followed by a sudden increase in sedimentation rate as the gel structure rapidly collapses. The final stage is the slow compaction of the sediment as it rearranges into a more compact structure.

\subsubsection{Measuring the Sedimentation Profile}

Figure 4.2 below shows schematically how the sedimentation profile can be built up by observing the suspension through time. Observations are made with a CCD camera and recorded onto video tape using a time-lapsed video recorder. The height of the suspension is then simply measured from a monitor with the time of the measurement given by the video recorder's own time signal which is displayed on the monitor.

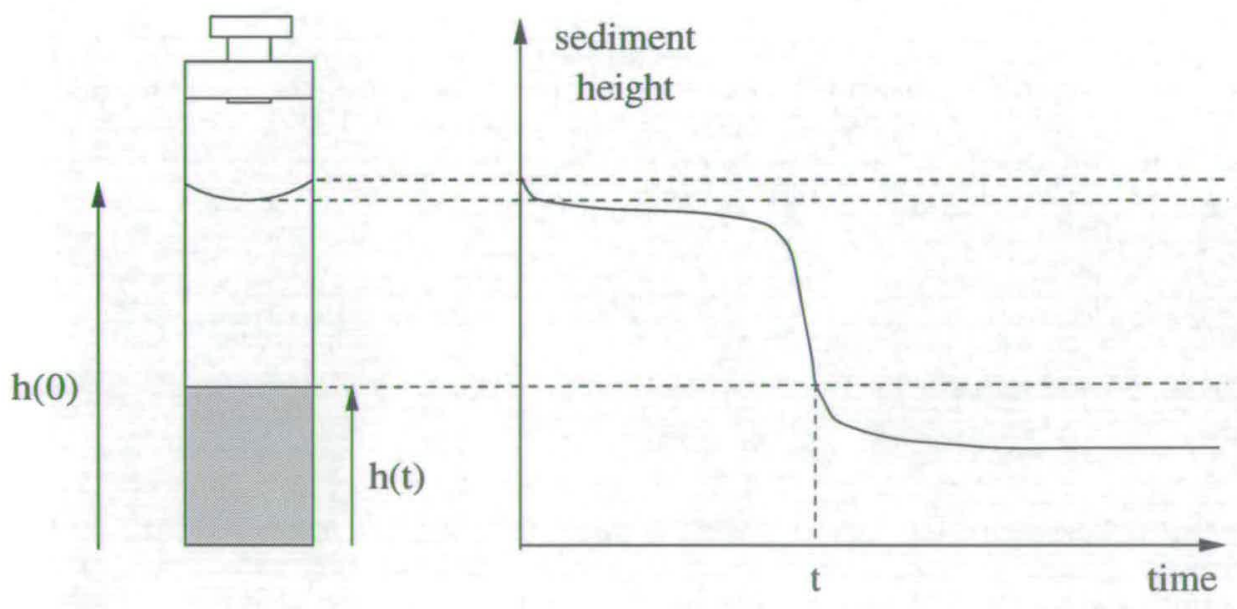

Figure 4.2: Schematic diagram showing how to produce a sedimentation profile. The initial suspension height $h(0)$, the height of the meniscus base, and the height at some later time $h(t)$ are shown.

The height of the suspension is measured along the outside edge of the sample cell. This means that the height at time zero, $h(0)$ on figure 4.2 , refers to the height of the suspension at the corner of the sample. This has the effect of producing a small tail at the beginning of the sedimentation profile caused by meniscus drainage. This tail becomes smaller as we make the sample taller or wider. For wide samples this is due to wider samples having a meniscus with lower curvature thus reducing the volume of the corners where drainage occurs. In tall samples the effect of the meniscus as compared with the bulk of the sample is relatively small and so the tail caused by drainage will also be small. 
One final point to mention is the magnification factor introduced by measuring height data from a large T.V. screen. The image produced by the direct observation setup as described in section 3.2 fills as much of the CCD array of the camera as possible. To do this requires a reduction in image size by the DOR. However, upon viewing the images on a T.V. monitor we observe an effective magnification since the image now fills the entire screen which is considerably larger than the CCD array.

It is desirable to have sedimentation data plotted as real suspension height against time so the measured data is reduced by a magnification factor $M=H(0) / h(0)$, where $H(0)$ is the initial sample height as measured on the screen at time zero and $h(0)$ is the real initial sample height.

\subsubsection{Extracting the Delay Time and Settling Velocities}

The sedimentation profile of a collapsing gel provides us with a simple way to define its delay time. We notice that the relationship between sediment height and time is almost linear in the delay and rapid collapse stages. If we fit two straight lines through the measured points, see figure 4.3 , then we find that the time coordinate of their intersection gives a good measure of the delay time of the gel. The gradients of each line give the settling rates in the delay and rapid collapse stages, $u_{s}$ and $u_{f}$ respectively.

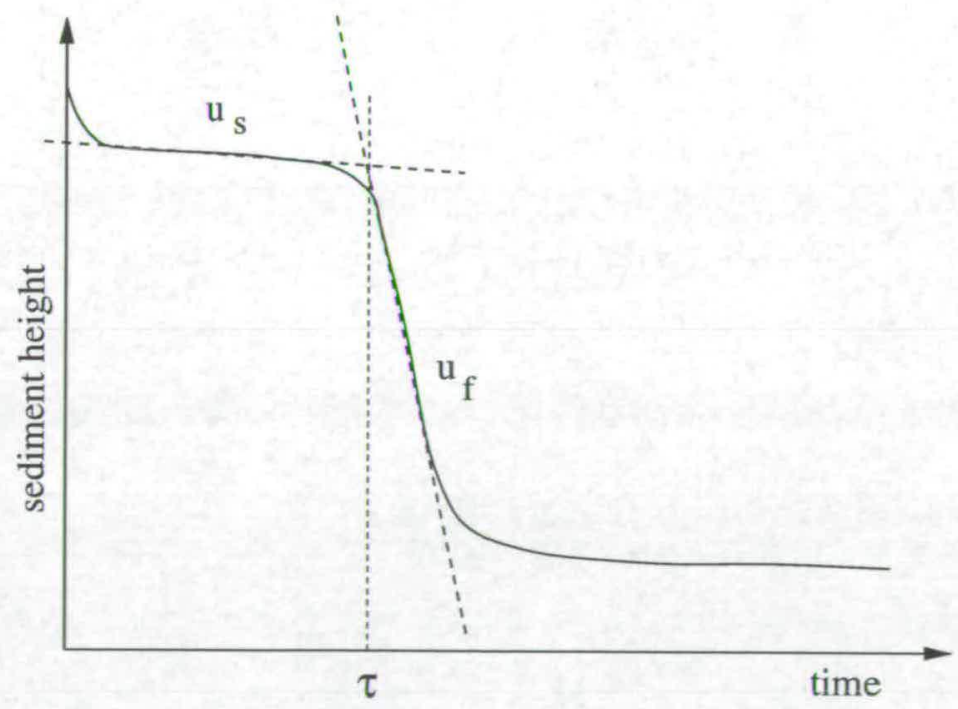

Figure 4.3: Schematic diagram showing how to measure the delay time, $\tau$, and the slow and fast settling rates, $u_{s}$ and $u_{f}$ respectively, from a sedimentation profile 


\subsubsection{Errors}

The error on the time measurements ( $\sim 0.1$ seconds) are essentially zero as they are insignificant compared with the length of the experiment (24 hours-weeks) and the time between measurements ( 5 minutes -5 hours)

The on-screen height of the suspension can be measured to an accuracy of $\pm 1-2 \mathrm{~mm}$ depending on the sharpness of the boundary being measured and the quality of the video recording. With a typical height measured varying from $50 \mathrm{~mm}$ to $100 \mathrm{~mm}$ this leads to a relative error of $\pm 1 \%-4 \%$ in the on-screen height $H$.

The real sediment height is found by dividing the on-screen height by the magnification factor. The magnification factor is found by dividing the on-screen initial height (typically $\Delta H(0)= \pm 2 \%)$ by the real initial height $(\Delta h(0)= \pm 1 \%)$. Thus the relative error on the magnification factor is typically $3 \%$.

Combining the error on the measured height with that on the magnification we calculate a typical relative error on the real sediment height of $\Delta h(t) \approx 5 \%$.

The error in determining the delay time from the sedimentation profile is estimated at $\Delta \tau= \pm 30$ minutes. This error is small when compared with the variation found in the delay time caused by random processes intrinsic to the gel. It has been found that the delay time of any particular gel sample can vary from experiment to experiment by $20 \%$ or more due to such processes. It is for this reason that all delay times are averaged over three measurements of the same sample.

In order to estimate the error on $u_{s}$ and $u_{f}$ we have calculated the standard deviation in the velocities measured from each experiment.

\subsection{Delayed Sedimentation Observations}

We shall concentrate our discussion on a set of samples with volume fraction $\phi \approx 0.2$ and varying polymer concentrations. However, we note that similar behaviour to that described here has been observed at higher and lower volume fractions. Table 4.1 gives the compositions $\left(\phi, C_{p}\right)$, free volume polymer concentrations $\left(C_{p}^{f r e e}\right)$, typical 
delay times $(\tau)$, and the average slow and fast settling rates $\left(u_{s}, u_{f}\right)$ for the samples discussed in this section. $C_{p}^{f r e e}=C_{p} / \alpha$ where $\alpha$ is the free volume fraction as given in equation 2.6.

\begin{tabular}{|c|c|c|c|c|c|}
\hline$\phi$ & $C_{p}\left(\mathrm{mgcm}^{-3}\right)$ & $C_{p}^{f r e e}\left(\mathrm{mgcm}^{-3}\right)$ & $\tau$ (minutes) & $u_{s}\left(\mu \mathrm{ms}^{-1}\right)$ & $u_{f}\left(\mu \mathrm{ms}^{-1}\right)$ \\
\hline 0.19 & 5.67 & 7.58 & $\approx 200$ & 0.1 & 1.2 \\
0.20 & 5.94 & 8.08 & $\approx 460$ & 0.08 & 0.8 \\
0.20 & 6.44 & 8.77 & $\approx 1000$ & 0.06 & 0.7 \\
0.20 & 6.64 & 9.04 & $\approx 1200$ & 0.04 & 0.6 \\
0.20 & 6.98 & 9.50 & - & - & - \\
\hline
\end{tabular}

Table 4.1: Table showing the volume fraction $\phi$, polymer concentration $C_{p}$ in $\mathrm{mgcm}^{-3}$, free volume polymer concentration $C_{p}^{f r e e}$ in $\mathrm{mgcm}^{-3}$, the typical delay time $\tau$ and the slow and fast settling rates, $u_{s}$ and $u_{f}$, of the samples to be discussed in this chapter.

For polymer concentrations above $5.5 \mathrm{mgcm}^{-3}$ we observe delayed sedimentation behaviour. This is evident from the characteristic shape observed in the sedimentation profile, figure 4.4. The delay time depends upon the free volume polymer concentration in a non-linear fashion as shown in figure 4.5. Measured delay times range from around 2 hours up to 25 hours.

The slow and fast settling velocities, $u_{s}$ and $u_{f}$ respectively, do not appear to be strongly dependent upon polymer concentration although they both decrease with increasing $C_{p}$. Typically, $u_{s}$ is around ten times slower than $u_{f}$ but still ten times faster than the Stokes velocity of an individual particle which for this system is estimated to be $v_{\text {stokes }}=\frac{2 \Delta \rho g a^{2}}{9 \eta} \sim 0.01 \mu \mathrm{ms}^{-1}$. In estimating the Stokes velocity we have assumed the density difference between the particles and the solvent to be $\Delta \rho=\rho_{p m m a}-\rho_{s} \simeq$ $300 \mathrm{kgm}^{-1}, \mathrm{a}=200 \mathrm{~nm}$ and the solvent viscosity to be $\eta \simeq 3 \times 10^{-3} \mathrm{~Pa}$ s.

At the highest polymer concentrations we find a different settling process which we call creeping sedimentation, figure 4.4 (purple down-triangles). These samples never undergo a transition to a rapidly sedimenting stage and we cannot define a delay time for them. The sample settles at a rate comparable with the slow settling rate of gels which exhibit delayed sedimentation behaviour. As the polymer concentration is increased further so the rate at which the samples creep slows down. 


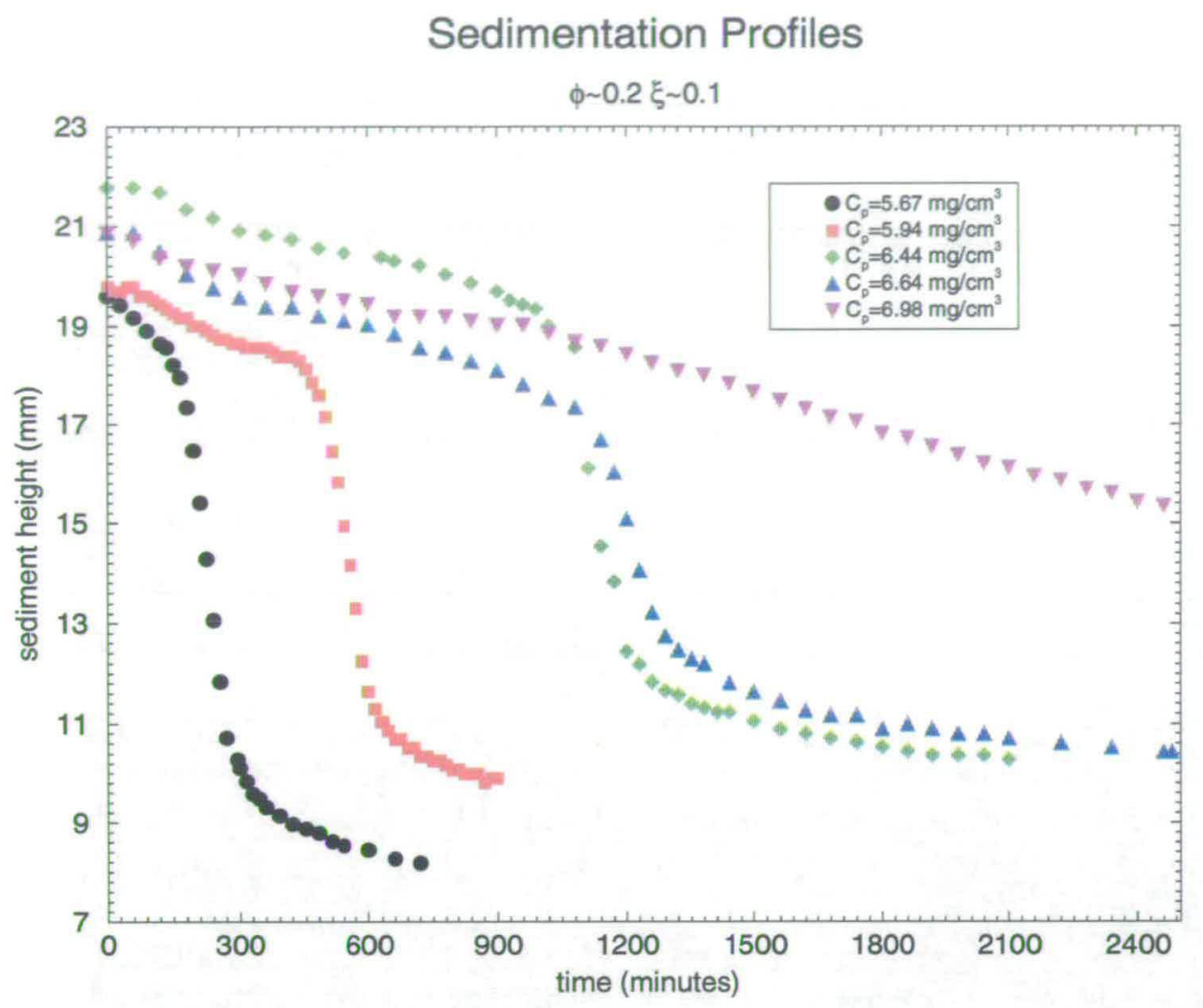

Figure 4.4: Sedimentation profiles of the samples discussed in this section (black circles $C_{p}=5.67 \mathrm{mgcm}^{-3}$, red squares $C_{p}=5.94 \mathrm{mgcm}^{-3}$, green diamonds $C_{p}=6.44 \mathrm{mgcm}^{-3}$, blue up-triangles $C_{p}=6.64 \mathrm{mgcm}^{-3}$, purple down-triangles $C_{p}=6.98 \mathrm{mgcm}^{-3}$.

\subsubsection{Short and Intermediate Delay Times}

At moderate polymer concentrations, $5.00 \mathrm{mgcm}^{-3} \lesssim C_{p} \lesssim 6.00 \mathrm{mgcm}^{-3}$, we observe gels which undergo delayed sedimentation with delay times ranging from 1 to 10 hours.

Figure 4.6 shows a time-lapsed sequence of dark-field images of a collapsing gel with a delay time of $\tau=460$ minutes ( $\sim 7$ hours). The sample shown here had a colloid volume fraction of 0.2 and polymer concentration $5.94 \mathrm{mgcm}^{-3}$. The initial height of the suspension was $24.5 \pm 0.1 \mathrm{~mm}$. The time at which images (a) to (l) were captured is shown on the sedimentation profile at the bottom of figure 4.6. In these pictures the very bottom of the sample has not been captured. Images (m)-(o) show a different gelled sample $\left(\phi=0.19, C_{p}=5.67 \mathrm{mgcm}^{-3} \tau \sim 200\right.$ minutes) where the initial suspension height was small enough to image the whole sample $(h(0)=10 \mathrm{~mm})$. 


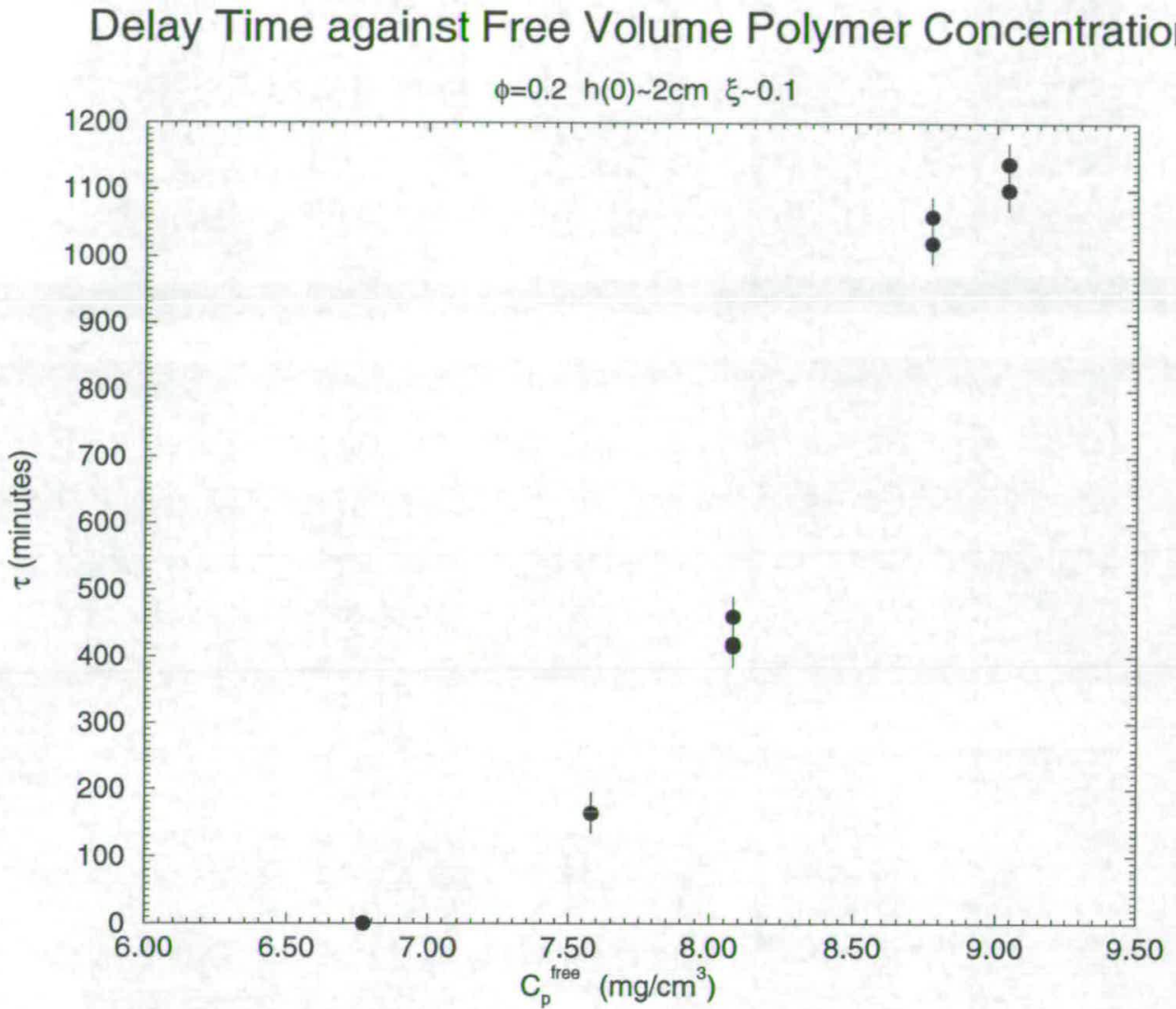

Figure 4.5: Plot of delay time against free volume polymer concentration for a sequence of gels with $\phi \approx 0.2$ and initial sample height $\mathrm{h}(0)$ of around $2 \mathrm{~cm}$.

Initially the whole sample appears to be dark except for small bright spots caused by dust on the inner surface of the sample cell, figure 4.6(a). During the delay period bright patches begin to form underneath the sample meniscus, figure 4.6(b), and grow across and downwards into the suspension. At the same time a layer of bright material begins to form at the bottom, figure $4.6(\mathrm{~m})$. This grows steadily throughout the delay time, figure $4.6(\mathrm{n})$, and reaches a maximum thickness of around $1 \mathrm{~mm}$.

Later on during the delay "channels" are observed travelling upwards in the sample. Figure 4.6(c) shows a channel that has just formed near the left hand wall of the sample cell. Channels also appear bright and are observed to start at various points within the sample. Some observations have shown a tendency for channel formation to begin at or near the cell walls suggesting that they may nucleate on small inhomogeneities on the inner cell wall. More than one channel is often observed as is shown in figures $4.6(\mathrm{~d})$ and $4.6(\mathrm{e})$ where two fainter channels can be seen to the right of the first one. 


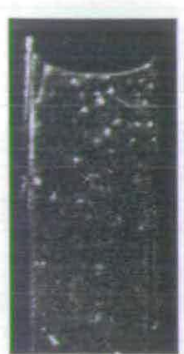

(a)

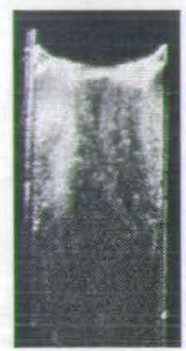

(f)

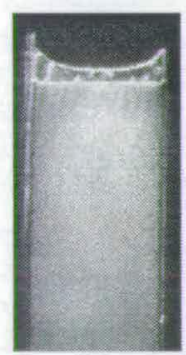

(k)

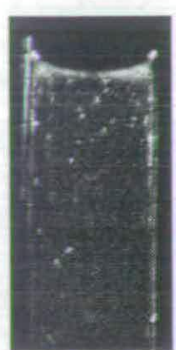

(b)

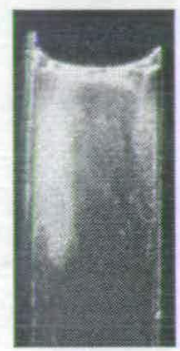

(g)

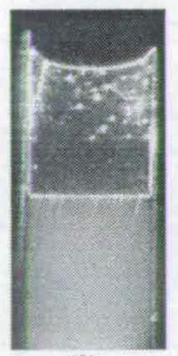

(1)

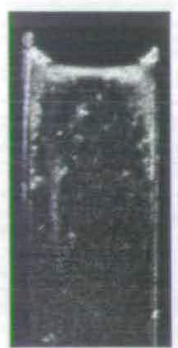

(c)

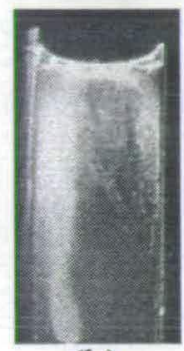

(h)

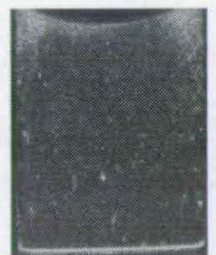

(m)

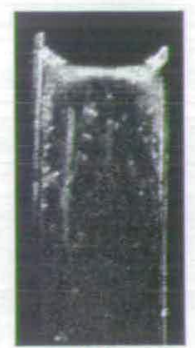

(d)

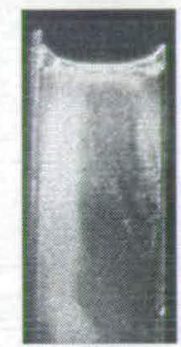

(i)

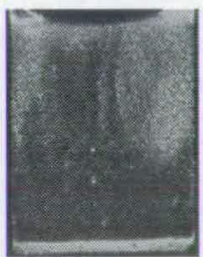

(n)

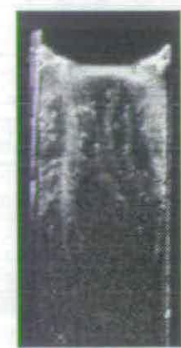

(e)

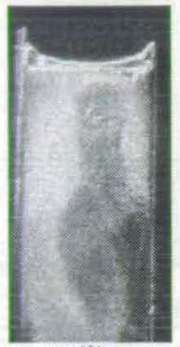

(j)

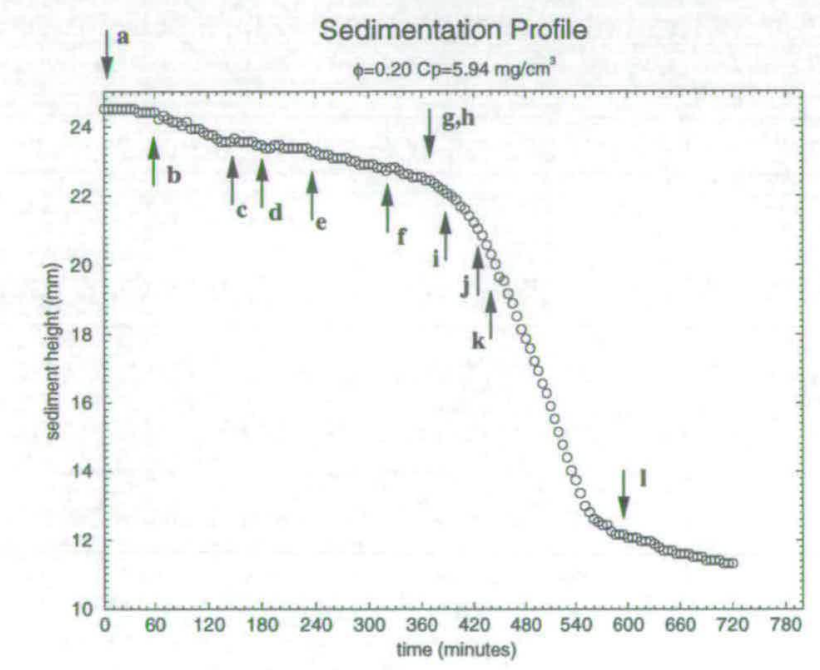

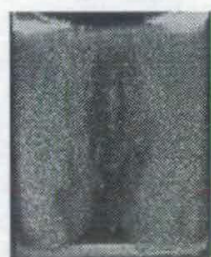

(o)

Figure 4.6: Dark-field images of a gel undergoing delayed sedimentation with a latency time of 420 minutes. For images (a)-(I) the time at which each image was captured is shown in the $h(t)$ plot for this sample. Images $(m)-(0)$ show what happens at the bottom of a sample during delayed sedimentation. Here the latency time is 200 minutes and the images were captured at 60,150 , and 180 minutes respectively. 
When a channel breaks through the surface of the suspension we observe small volcanolike structures as colloidal material carried by the channel is deposited. Once a channel breaks the surface of the suspension it begins to grow in width. The typical width of a channel initially is of order $0.5 \mathrm{~mm}$ which can increase to $2-3 \mathrm{~mm}$ before the onset of rapid collapse.

Approximately half way through the delay, the sedimentation of the suspension surface becomes quite noticeable, figure 4.6(e,f). The bright patches now extend a considerable distance below the suspension surface $(\sim 1 \mathrm{~cm})$ and form what are called "streamers". These streamers sink rapidly into the suspension, figure $4.6(\mathrm{~h})-(\mathrm{i})$, triggering the rapid settling of the gel. Upon reaching the bottom, the streamers destroy the layer of bright material by mixing, figure $4.6(\mathrm{o})$.

Streamers have a tendency to travel down the paths formed by earlier channels, figure 4.6(f)-(h). This is not always true as can be seen in figure 4.6(n) where a wide channel has broken through the suspension surface near its middle while the streamers still propagate from the upper corners of the sample.

Small volcano-like features are observed on the suspension surface above the streamers. These form due to the expulsion of solvent from the suspension which is causing some of the material on the surface to be forced upwards into the supernatant. The size and frequency of these volcano-like features rises dramatically moments before the streamer velocity suddenly increases indicating an increase in solvent expulsion from the suspension.

During the rapid sedimentation phase, figures $4.6(\mathrm{k})-(\mathrm{l})$, circulation of material can be seen (not visible in still pictures). During this part of the settling process the fluctuation of speckles is very rapid and slows down as the suspension settles. These speckles are caused by the partially coherent nature of the illumination source and are not a feature of the sediment.

A region of slow speckle fluctuation is observed to grow upwards from the bottom of the cell during this time indicating the presence of dense amorphous sediment forming at the bottom as the suspension settles. A diffuse boundary between settling and compact suspension can be observed and measured. When the top sediment surface 
and the bottom compaction surface meet the rate of settling falls dramatically. The gel is now in the slow sedimentation or compaction stage.

\subsubsection{Long Delay Times}

Dark-field observations of delayed sedimentation in gels with long delay times show similar features to those of the shorter lived gels. The formation of channels, bright bands and streamers are all observed. However, we also observe horizontal cracking of the suspension during the delay time. This can occur in the bulk region of the sample but frequently starts from the cell walls and propagates inwards and upwards into the sample. These features are illustrated in figure 4.7 which shows a sequence of dark-field images captured during the delay stage from a gel with a delay time of 1000 minutes.

Figure 4.7(a) shows a crack that has just formed on the left hand side of the sample at approximately one third of the total sample height. This crack propagates into the sample as shown in figures 4.7 (b) to 4.7 (d). The very bright areas near the top of the samples indicate regions where many small cracks have formed. This region was so bright that it was difficult to see the formation of streamers although they were observed. Figure 4.7(c)-(e) show a channel moving upwards in the sample from an origin near the base. We also observe a bright band growing in thickness near the bottom. In this case the band was much brighter and reached a larger maximum thickness than was observed for shorter lived gels. The layer of bright material persisted after the onset of rapid collapse.

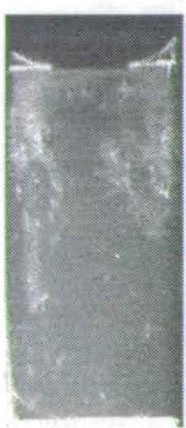

(a)

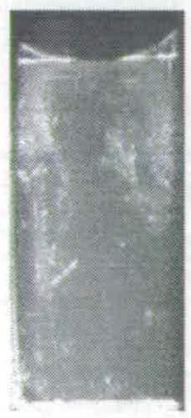

(b)

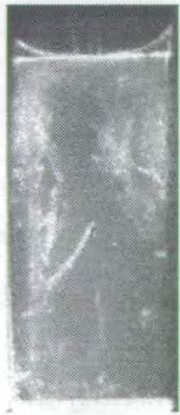

(c)

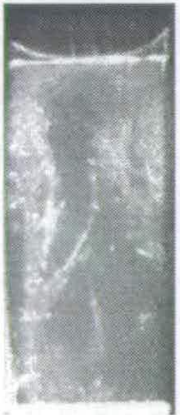

(d)

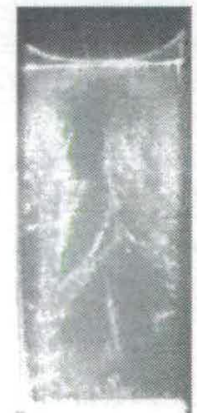

(e)

Figure 4.7: Dark-field images of a gel exhibiting delayed sedimentation with a delay time of 1000 minutes. Images (a) to (e) were captured at $360,420,480,540$, and 600 minutes respectively. The initial sample height is $h(0)=20.8 \mathrm{~mm}$. 
It has already been shown that the fast settling rate $u_{f}$ slows down as we increase the polymer concentration and therefore the delay time. We also observe a slowing down of streamer propagation rate. During the rapid collapse stage the circulation of material also appears slower than in the shorter lived gels.

\subsection{Creeping Sedimentation Observations}

At the highest polymer concentrations there is a sudden change in the settling behaviour as illustrated by the sedimentation profile, figure 4.4. Creeping sedimentation behaviour has been observed in a previous study of gel collapse [16] but was never studied in detail.

Figure 4.8 shows a time-lapsed sequence of dark-field images for a sample which exhibits creeping sedimentation behaviour $\left(\phi=0.20, C_{p}=6.98 \mathrm{mgcm}^{-3}\right)$. Initially the sample appears dark except for small bright spots caused by dust on the cell walls. The suspension begins to slowly sediment almost immediately after the beginning of the experiment. A coarse, bright texture forms at the top of the sample at early times, figure $4.8(\mathrm{c})$, and moves downwards with time. Further down in the suspension, cracklike features grow inwards and upwards into the sample from the cell walls from $t=14$ hours onwards, figure 4.8(e)-(h). These features move very slowly into the sample and are similar in appearance to those observed in long delay times gels, see figure 4.7. The cracks first appear near the top of the sample, figure 4.8 (d)-(e), but as the suspension settles, they begin to form lower down 4.8(f)-(i). Smaller cracks are also observed propagating horizontally far from the cell walls, e.g. figure $4.8(\mathrm{~d})-(\mathrm{f})$.

The cracks are finally broken up by the downward propagating bright texture, figure $4.8(\mathrm{j})-(\mathrm{m})$, which eventually fills the entire sample cell. These samples have been observed over a period of weeks and have shown no sign of rapid collapse. Samples with higher polymer concentrations show creeping behaviour but with slower settling rates.

Visual inspection of the bright region shows a crumbled texture as if it were composed of large aggregates. Large cracks open up in the sediment producing voids which are then filled by surrounding material. In the regions where no cracking is observed the sample appears smooth and unperturbed. 


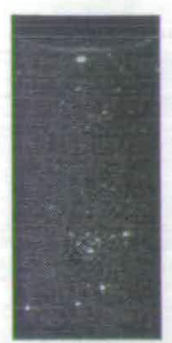

(a)

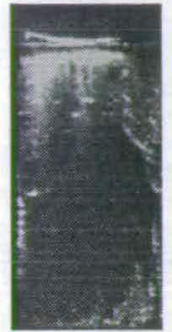

(f)

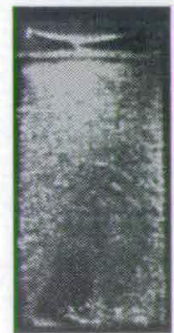

(k)

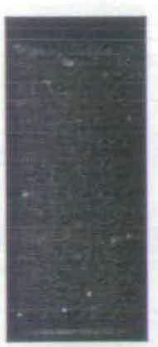

(b)

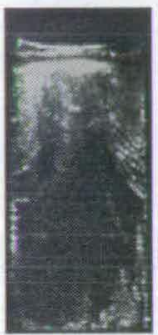

(g)

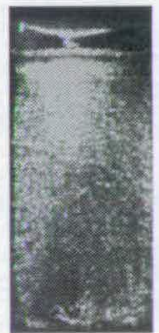

(1)

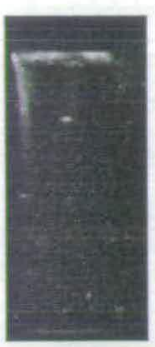

(c)

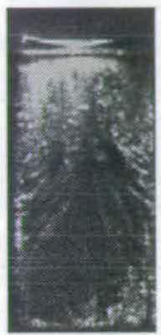

(h)

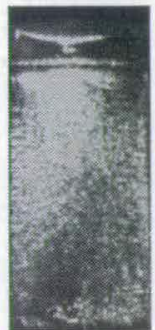

(m)

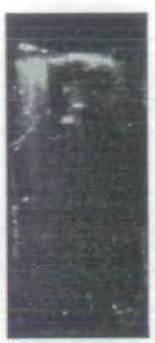

(d)

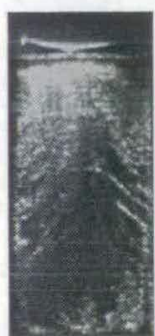

(i)

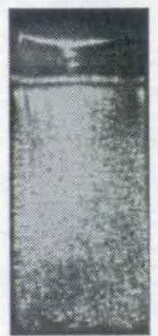

(n)

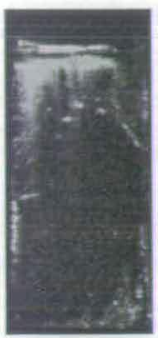

(e)

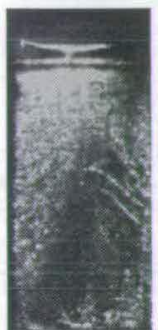

(j)

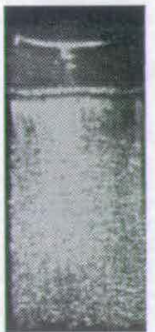

(o)

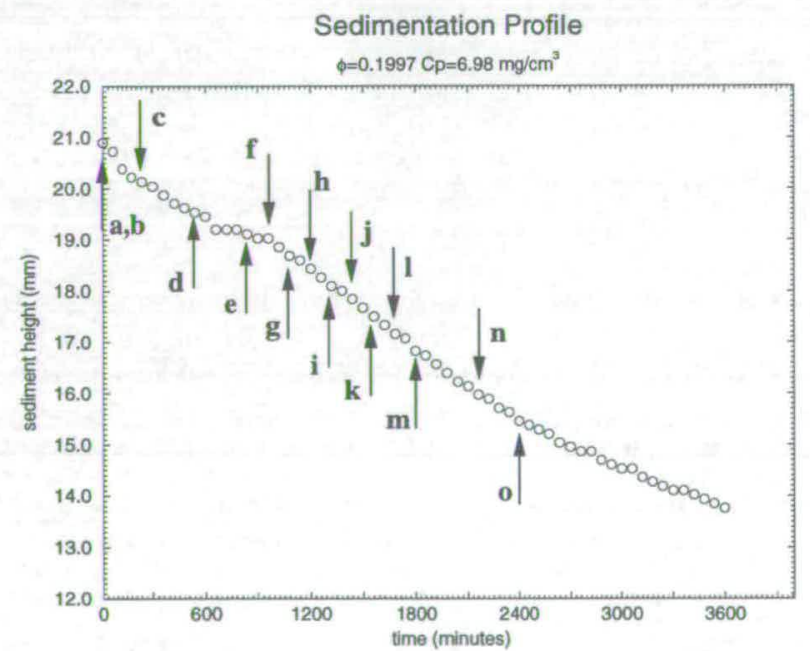

Figure 4.8: Dark-field images of a gel exhibiting creeping sedimentation behaviour. The time at which each image was captured is shown on the $h(t)$ plot. 


\subsection{Discussion}

We now make some comments on the results of our observational study of gel collapse. It is clear from our observations that there is no difference in the way in which a long delay time gel and a short delay time gel collapse, i.e. there is no observable difference between strong and weak gels. The features observed in collapsing gels are the same over a wide range of delay times with the only difference being a slowing down of the rate of streamer propagation and circulation of material at higher $C_{p}$.

Many of the comments made here will be revisited when we present a more general discussion of gel collapse in chapter 7 .

\subsubsection{Channels}

Channels are direct routes through which solvent can reach the suspension surface. As the suspension slowly settles during the delay time solvent is forced upwards. Rather than diffusing through the interconnected pores of the gel structure, solvent prefers to create channels through which it can flow more easily. In creating a channel the solvent causes local breaking of the gel structure which is why channels appear bright in the dark-field images.

Figure 4.9 shows the variation of channel-front height with time for four different channels for a gel with a delay time of 420 minutes. The data is taken from two separate experiments run under the same conditions (temperature, initial suspension height, mixing history, etc.).

From figure 4.9 it is clear that some measurements give a more complete record of the channel development than others. For example, curves 2 and 4 are similar to curves 1 and 3 but some of the early time data is missing. This is due to the fact that the exact point of origin for any individual channel is not always observed as this point can be out of the plane of focus.

The main feature of these curves is their apparent similarity in shape irrespective of the initial height and time. This is shown more clearly in figure 4.10 which shows the same curves but shifted in height and time in order to make them collapse onto one 


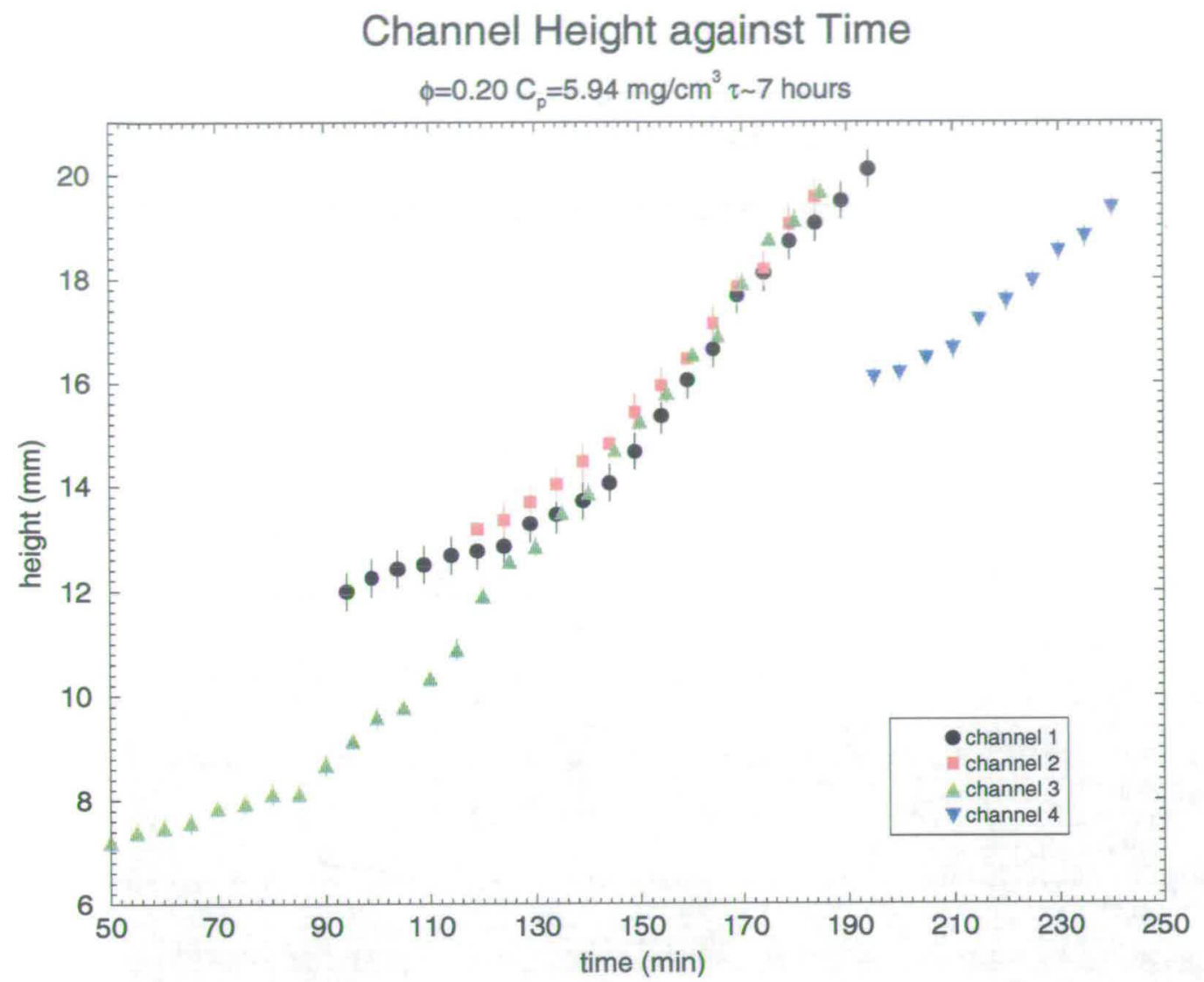

Figure 4.9: Graph of channel height against time for a gel showing delayed sedimentation with delay time $\tau \approx 420$ minutes $\left(\phi=0.20, C_{p}=5.94 \mathrm{mgcm}^{-3}\right)$.

curve. To form this plot, the curves are first shifted by a height and time constant so that they all have a common origin at $h=0 \mathrm{~mm}, t=0 \mathrm{~min}$. Curves 1,2 , and 4 are then further shifted relative to curve 3 to account for an out-of-focal-plane point of origin. From the observations we are confident that the origin of curve 3 was observed in the focal plane.

The channel propagation speed suddenly increases 50 minutes after it has formed as if a yield stress of the material has been overcome. By measuring the average slope of the channel height against time curves we have estimated the slow and fast propagation rates for the channels. The fast propagation rate of the channel $u_{f}^{c}=2.1 \mu \mathrm{ms}^{-1}$ is of the same order of magnitude as the fast sedimentation speed $u_{f}$ observed once the gel has begun to rapidly collapse. The slow channel propagation rate $u_{s}^{c}=0.5 \mu \mathrm{ms}^{-1}$ is ten times faster than the slow sedimentation rate 


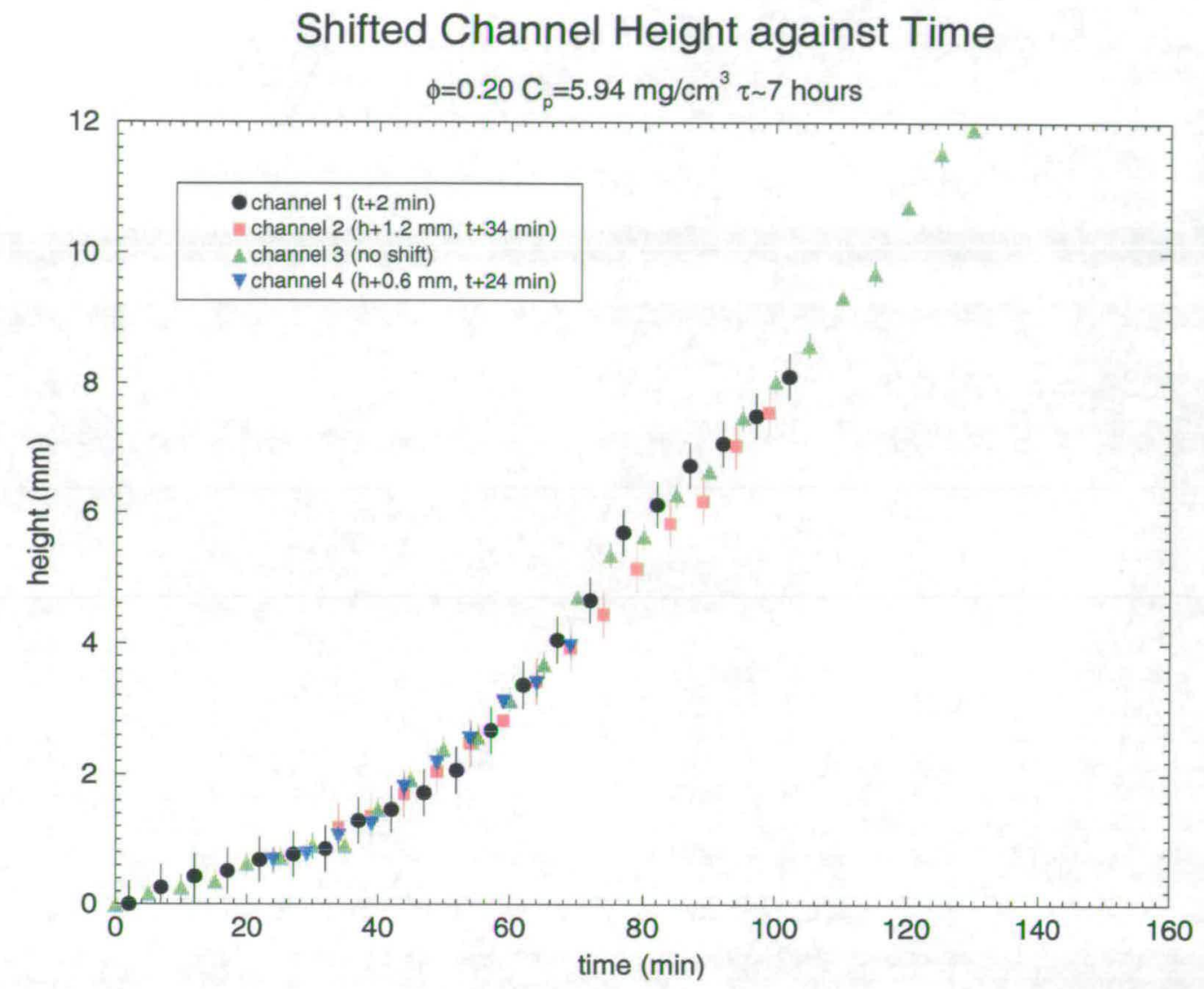

Figure 4.10: Graph of shifted channel height against time for a gel showing delayed sedimentation with delay time $\tau \approx 420$ minutes $\left(\phi=0.20, C_{p}=5.94 \mathrm{mgcm}^{-3}\right)$.

\subsubsection{Streamers}

Figure 4.11 shows how the height of a streamer-front varies with time in a sample with $\phi=0.2, C_{p}=5.94 \mathrm{mgcm}^{-3}, \tau \approx 420$ minutes. The rate at which the streamers move through the sample increases rapidly as the end of the latency time approaches. Streamers initially move relatively slowly through the sample, however at around $t=348$ minutes the rate at which the streamer propagates suddenly increases signalling the onset of rapid collapse. This is another example of yield-like behaviour in transient gels.

The slow propagation velocity as determined from figure $4.11\left(u_{s}^{s t}=1.7 \mu \mathrm{ms}^{-1}\right)$ is of the same order of magnitude as the fast sedimentation rate $u_{f}$. The streamer's fast propagation velocity is ten times faster than the fast sedimentation rate. 


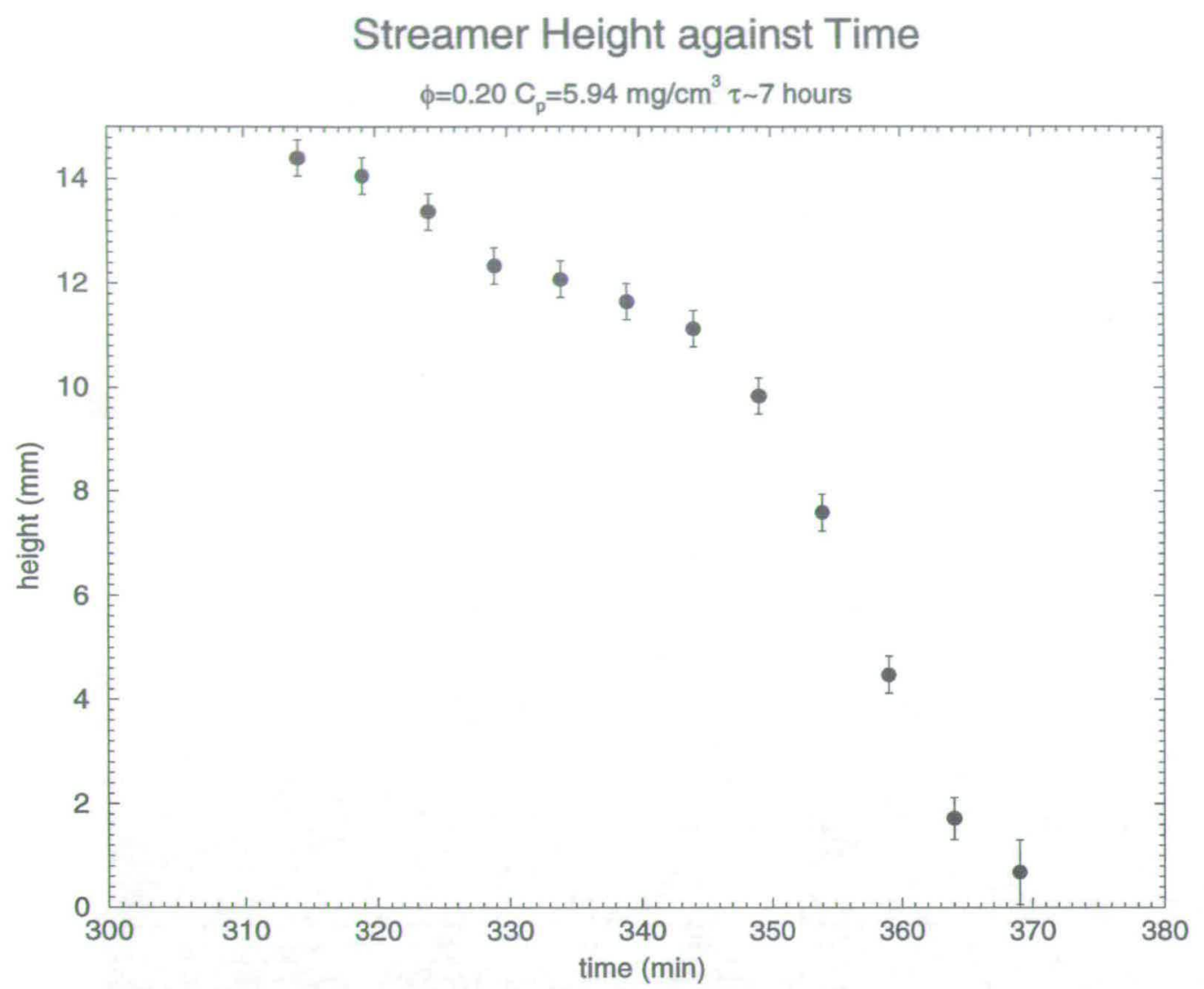

Figure 4.11: Graph of streamer height against time for a gel showing delayed sedimentation with delay time $\tau \approx 420$ minutes $\left(\phi=0.20, C_{p}=5.94 \mathrm{mgcm}^{-3}\right)$.

The formation of streamers is due to local breaking of the gel structure near the top of the suspension. Broken gel shows up as bright regions in our dark-field technique. The local breaking of the network produces regions of more compact and therefore denser clusters which we observe as bright patches. These more dense regions will then sink through the gel causing more local flow and thus allowing the material to sink faster due to the shear-thinning properties of the gel.

\subsubsection{Bright Bands}

The growth of a layer of bright material at the bottom of a gel indicates the presence of structural rearrangement due to the slow settling of the bulk. Figure 4.12 shows a plot of band thickness against time for a gel with delay time $\tau \approx 420$ minutes $(\phi=$ 
$0.20, C_{p}=5.94 \mathrm{mgcm}^{-3}$ ). From this plot we see that the band of bright material grows linearly in time until its destruction by the streamers.

\section{"Compact" Layer Height against Time}

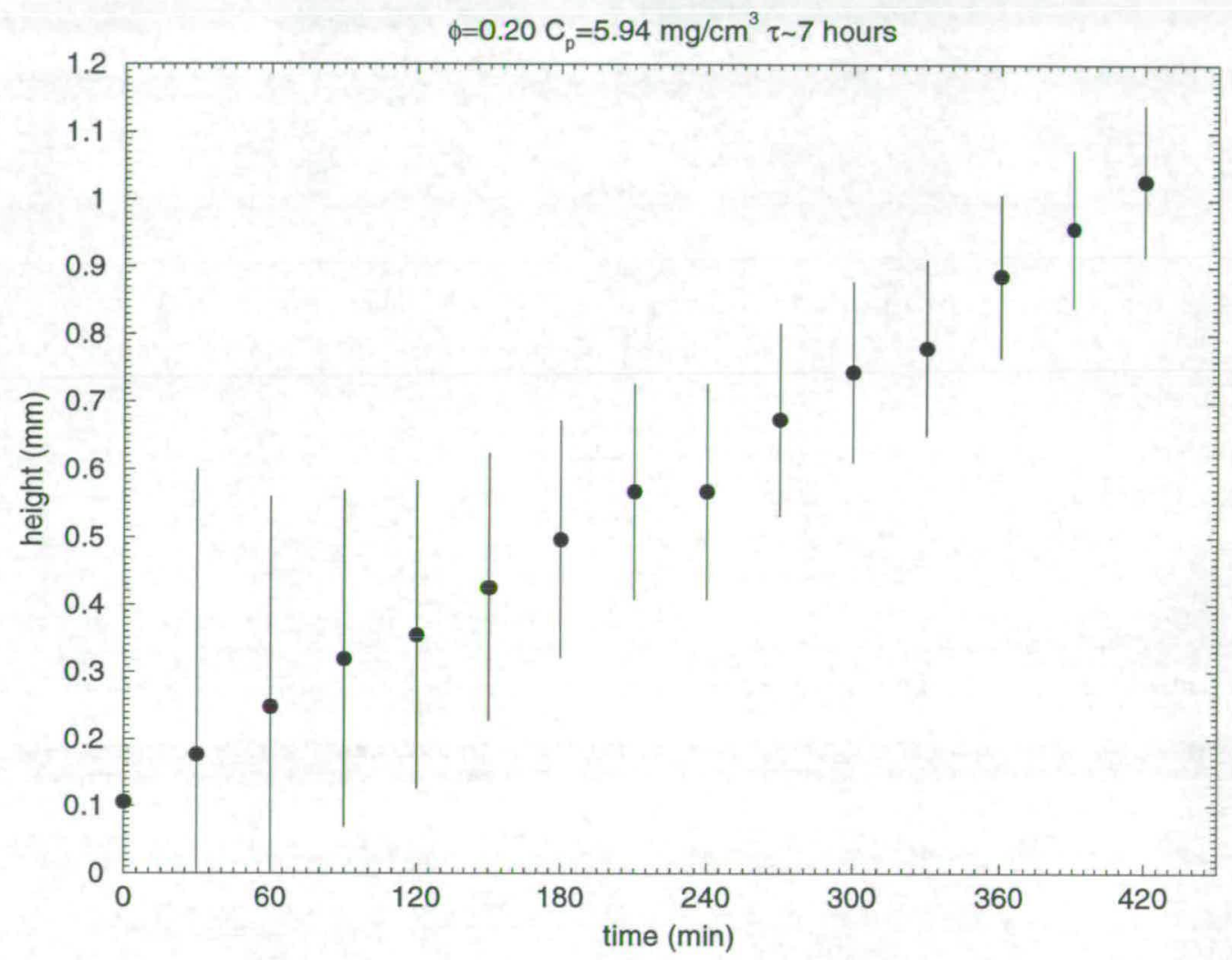

Figure 4.12: Graph of thickness of the layer of bright material against time for a gel with delay time $\tau \approx 7$ hours $\left(\phi=0.20, C_{p}=5.94 \mathrm{mgcm}^{-3}\right)$.

Observations at higher magnification, figure 4.13, show some texture in these bright layers which is different from the speckled texture seen in streamers and channels. No change in the texture with time has been observed. The boundary between the bright layer and the rest of the suspension is fairly well defined.

The brightness of these bands suggests that they are made of a more compact structure than the rest of the gel structure. This may be produced by crushing of the gel base as solvent is expelled from this region due to slow settling of the suspension during the delay time. 


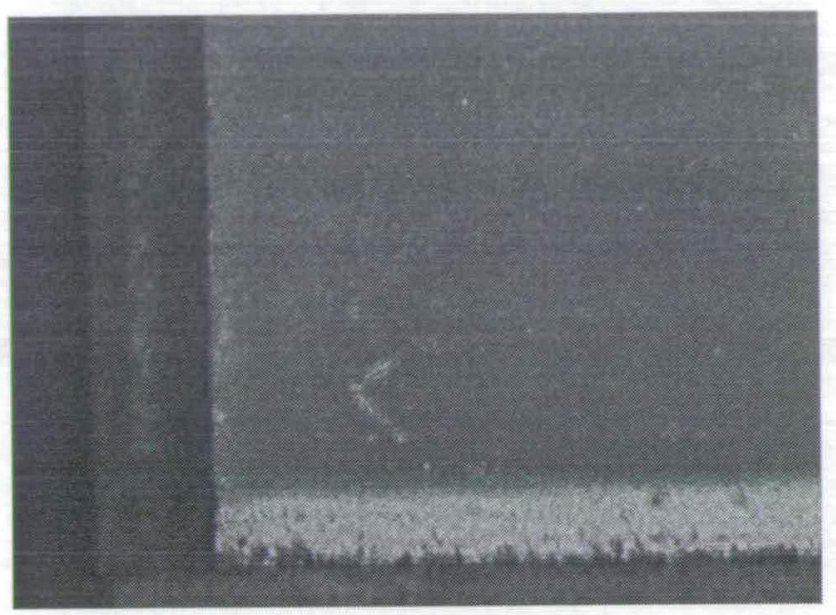

Figure 4.13: Close up of the bottom $3 \mathrm{~mm}$ of a gel with delay time $\tau \approx 3$ hours $(\phi=$ $0.20, C_{p}=5.64 \mathrm{mgcm}^{-3}$ ) showing the layer of bright material. The layer is around $0.5 \mathrm{~mm}$ in thickness.

\subsubsection{Cracks}

The appearance of crack propagating from the cell walls in long lived gels and samples exhibiting creeping sedimentation suggest an increasing importance of wall stress in supporting the suspension as polymer concentration increases. The importance of structural support from the walls will be discussed in more detail in chapter 6 .

\subsubsection{Creeping Sedimentation}

We have shown that suspensions exhibiting creeping sedimentation behave in a different manner to those which undergo rapid collapse. The reason for a change in settling behaviour is not clear from observations alone. However, we note that samples which display creeping behaviour never form streamers which are known to trigger the onset of rapid collapse. It is clear that an alternative method of probing gel sedimentation is required in order to formulate some model of this behaviour. Such a study is the topic of the next chapter. 


\section{Chapter 5}

\section{Concentration Profiles}

\subsection{Introduction}

Direct observation of gels has given us some insight into the physical processes involved in the collapse process. In the images presented we interpret regions of brightness to indicate broken gel structure. This implies that these regions are denser than the surrounding gel and can cause instability to propagate throughout the whole sample, e.g. streamer formation leads to global mixing of the gel in figure 4.6. Using a non-intrusive ultrasonic technique [31] it is possible to measure the concentration of a colloidal suspension. We now present the results of such a study and discuss them in the context of what is already known from observation.

The experiments presented in this chapter were carried out at the Institute of Food Research, Norwich, and the author wishes to acknowledge the help and advice of Dr. Margaret Robins and Dr. David Hibberd in performing the experiments and analysing the data.

\subsection{The Model System}

The system studied in this chapter is the same PMMA-PS mixture as described in section 4.2 but the suspending solvent used is pure cis-decalin. The suspending solvent has been changed for two reasons. Firstly, the experimental technique does not require 
index-matched samples and secondly, it is found that tetralin significantly damages the sample cells used in the ultrasonics experiments.

The particles have mean radius $a=186 \mathrm{~nm}$ with a polydispersity of $5 \%$. The polymer has molecular weight $M_{w}=320,000 \mathrm{gmol}^{-1}$. At $25^{\circ} \mathrm{C}$ in cis-decalin the polymer has a radius of gyration of $r_{g}=17 \mathrm{~nm}$ (as calculated using the method set out by Berry [38]) giving a size ratio for this system of $\xi=0.09$.

\subsubsection{Sample Preparation}

A batch of PMMA colloidal particles was first prepared in cis-decalin. The colloid stock was calibrated by the centrifuging method described in section 4.3

The polymer stock was produced by placing a known mass of dry polystyrene in a container and then adding a known amount of cis-decalin by weight. The polymer stock was made up to a concentration of $C_{p s}=14.75 \mathrm{mgcm}^{-3}$ and then tumbled overnight to dissolve the polymer in the solvent completely.

Measured amounts of each component (colloid, polymer and solvent stock) were then added by weight in glass sample cells and mixed well to produce a sample of desired colloid volume fraction $\phi$ and polymer concentration $C_{p}\left(\right.$ in $\left.\mathrm{mgcm}^{-3}\right)$.

Samples were prepared in $30 \mathrm{ml}$ glass containers and slowly tumbled overnight until all the components were well mixed.

\subsection{Experimental Method}

The experimental method employed to measure sample concentration ultrasonically has already been described in section 3.3.

Once fully mixed the sample was carefully transferred to the ultrasonic sample cell and immediately placed in the temperature bath. The sample cell was usually filled to a height of around $8 \mathrm{~cm}$ in order to obtain as many points as possible on the concentration profile. The sample was then allowed to stand in the temperature bath at $25^{\circ} \mathrm{C} \pm 0.02^{\circ} \mathrm{C}$ for 45 minutes before the first ultrasonic measurements were made. This was to allow the sample to reach a steady temperature. 
The experimental set does not allow synchronous measurements of time-of-flight at all heights in the sample as there is only one pair of transducers which vertically scan the suspension. Therefore each point in the concentration plots is measured at a different time. In a sediment showing complicated behaviour it can take up to 30 minutes to finish a scan as we require many points to see the full picture and it is assumed that the suspension does not change significantly during this period. The time label given to a particular profile is the time at which the scan of the sample finishes. Each scan of the sample is made from the bottom to the top.

\subsection{Results}

The composition, initial sample height, and delay time for the samples presented in this section are shown in table 5.1. The settling behaviour of each sample was recorded by a video camera at the same time as the time-of-flight data was captured. This was used to generate a plot of sediment height against time from which the delay time was extracted in the manner described in section 4.4. The longest delay time sample was also used to produce creeping behaviour by reducing the initial height of the sample to $55 \mathrm{~mm}^{*}$.

\begin{tabular}{|c|c|c|c|}
\hline$\phi$ & $C_{p}\left(\mathrm{mgcm}^{-3}\right)$ & $h(0)(\mathrm{mm})$ & $\tau$ (hours) \\
\hline 0.19 & 7.39 & 79 & 3 \\
0.19 & 8.50 & 81 & 8 \\
0.19 & 10.66 & 71 & 25 \\
\hline
\end{tabular}

Table 5.1: Table of compositions and delay times for the samples studied by ultrasonic concentration profiling

The area under the concentration profile should remain constant with time since the mass of the system is conserved. This gives us a method to the accuracy of the Urick calibration by integrating the concentration against height data. For each of the samples presented in this section, we find a variance of $3 \%$ to $4 \%$ in the mean area under each curve.

\footnotetext{
*Height-dependency will be discussed in more detail in chapter 6
} 


\subsubsection{Short and Intermediate Delay Times}

Figure 5.1 shows a plot of colloid volume fraction against height in the suspension for a gel with a delay time of 3 hours. Each line in this plot represents the concentration profile measured at a different time. Initially the sample has a homogeneous concentration of $\phi \approx 20 \%$ as indicated by the black horizontal line. The rapid drop in concentration at the top of the sample is due to making measurements in the meniscus. Here, only part of the ultrasonic pulse passes through the sample while the other part passes through the air above the meniscus.

\section{Concentration Profile}

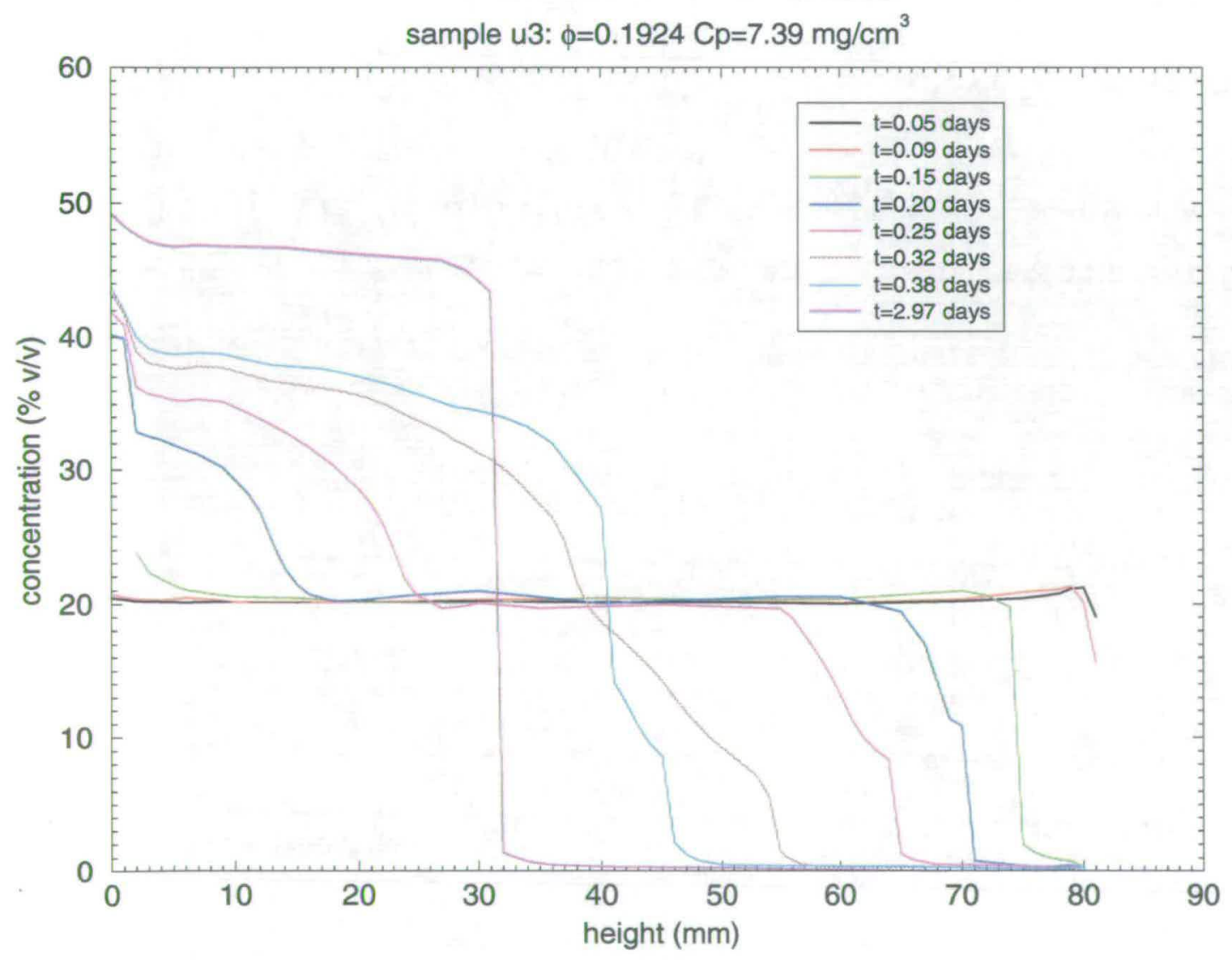

Figure 5.1: Concentration profile of a gel with a delay time of $\tau \approx 3$ hours ( 0.125 days). Each line represents the concentration profile measured at a different time (black $=0.05$ days, red $=0.09$ days, green $=0.15$ days, dark blue $=0.20$ days, magenta $=0.25$ days, brown $=0.32$ days, light blue $=0.38$ days, and purple $=2.97$ days).

The concentration profile remains unchanged throughout the delay time. Small rises in concentration are observed at the very top and bottom of the suspension. These 
features are difficult to see in short lived gels as they are small $(\sim 1 \%)$ but they are larger in gels with longer lifetimes as shown in figure 5.2 which shows the concentration profile for a gel with a delay time of $\tau=8$ hours.

By $\mathrm{t}=0.15$ days $(\approx 3.5$ hours $)$ the gel has started rapid collapse as shown by the green profile in figure 5.1. A sharp interface between the settling suspension and the supernatant left behind is clearly visible in this profile as a vertical line at a height of around $74 \mathrm{~mm}$. The concentration above this sharp interface falls to zero indicating that no colloidal material is left behind as the gel collapses. The concentration gradually increases towards the bottom of the sample while the bulk of the suspension remains homogeneous in concentration at the original volume fraction (20\%).

\section{Concentration Profile}

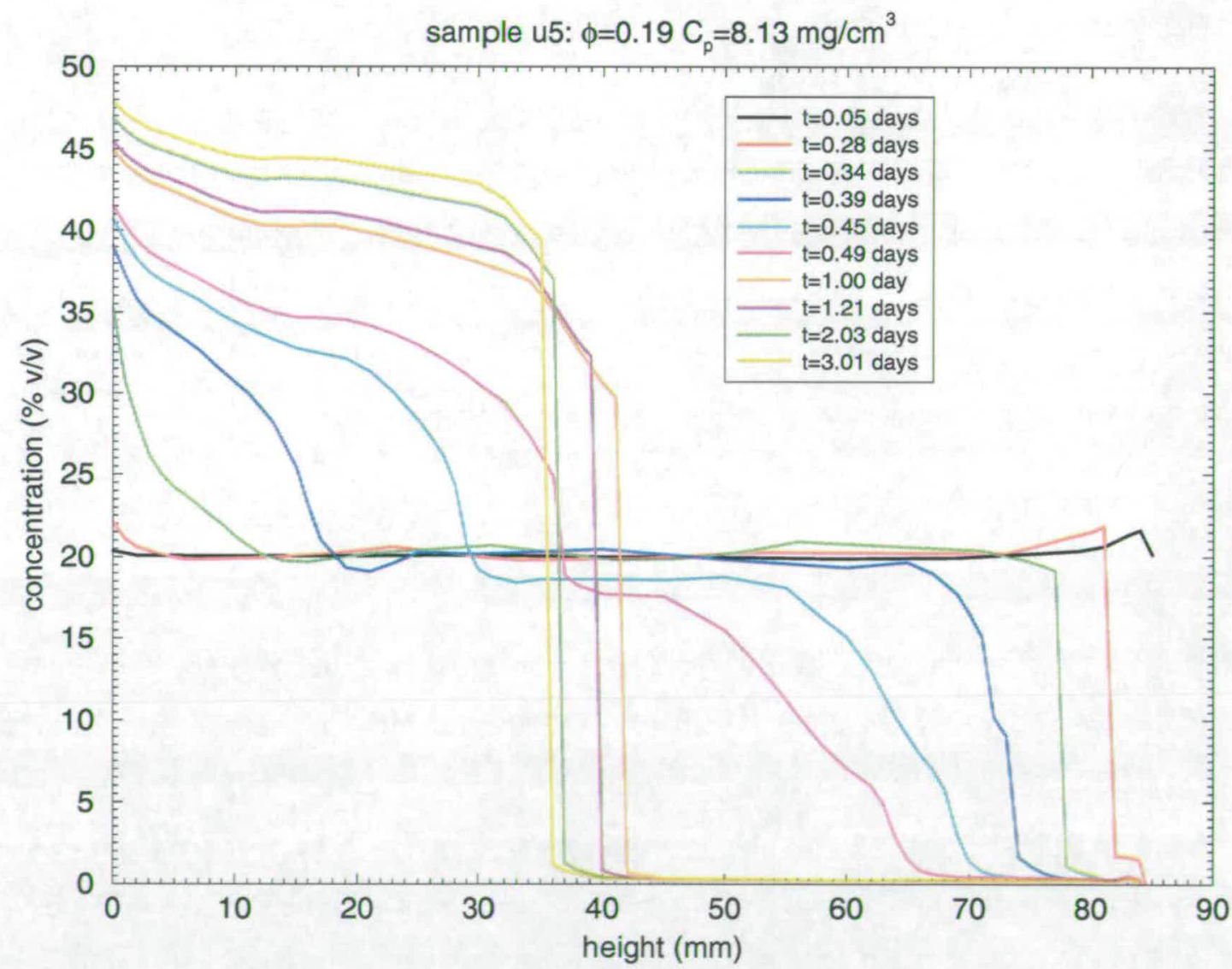

Figure 5.2: Concentration profile of a gel with a delay time of $\tau \approx 8$ hours ( 0.33 days). Each line represents the concentration profile measured at a different time (black $=0.05$ days, red $=0.28$ days, green $=0.34$ days, dark blue $=0.39$ days, light blue $=0.45$ days, magenta $=0.49$ days, orange $=1.00$ day, purple $=1.21$ days, dark green $=2.03$ days, and yellow $=3.01$ days) . 
As time progresses the top boundary continues to fall, $\mathrm{t}=0.20-0.25$ days (4.74-6 hours). Although this boundary remains sharp, we observe a gradient in concentration forming and growing underneath it. Immediately below this first region of concentration gradient there is a thick layer of material which remains at the original concentration. Lower in the suspension a layer of dense sediment grows upwards from the bottom. The sediment gradually decreases in concentration with increasing height.

The middle layer of constant concentration decreases in thickness as the suspension settles and by 0.32 days $(\approx 8$ hours) has disappeared. We now observe a sharp boundary at the top below which there is a layer about $16 \mathrm{~mm}$ in thickness where the concentration increases from zero to 20 percent as height decreases. Below this we observe a thick layer of dense sediment which gradually increases in concentration as height decreases. The interface between the dense sediment and less dense layer is very diffuse at first. However, as settling proceeds, $\mathrm{t}=0.38$ days $(\approx 9$ hours) this interface sharpens and moves upwards in the sample.

Eventually, after 3 days, there is no less dense layer left and we observe a very sharp interface between colloidal sediment and supernatant. Once the rapid collapse stage is over the sediment slowly compacts under its own weight. This can be seen in the profile of the gel with $\tau=8$ hours, figure 5.2 ( $\mathrm{t}=1-3$ days). The sediment gradually increases in concentration while the interface decreases in height. We also notice that the gradient in sediment concentration decreases during compaction.

\subsubsection{Long Delay Times}

Figure 5.3 shows the concentration profiles for a gel with a delay time of 25 hours. We observe many features similar to those observed for much shorter lived gels. The suspension is initially homogeneous at a volume fraction of around $20 \%$ and remains so throughout the delay time. We observe clear rises of up to $4 \%$ in concentration in the top and bottom few millimetres during the delay time while the bulk of the suspension remains unchanged. We also observe the formation of a sharp interface between colloid and supernatant at the top before the onset of rapid collapse signalling that this sample has settled below the base of the meniscus before it has started to rapidly collapse. 


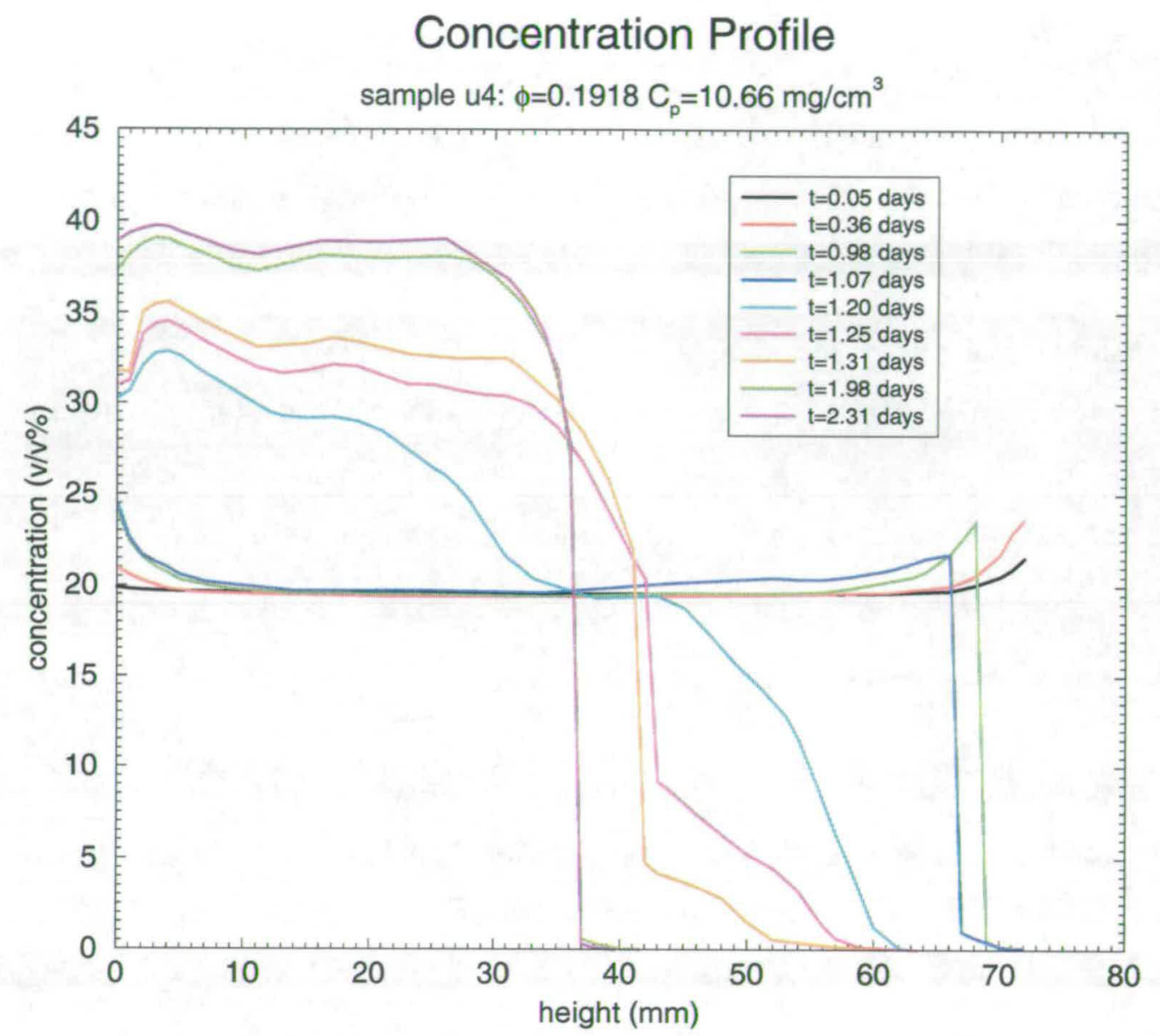

Figure 5.3: Concentration profiles of a long delay time gel, $\tau \approx 25$ hours. Each line represents the concentration profile measured at a different time (black $=0.05$ days, red $=0.36$ days, green $=0.98$ days, dark blue $=1.07$ days, light blue $=1.20$ days, magenta $=1.25$ days, orange $=1.31$ days, dark green $=1.98$ days, and purple $=2.31$ days).

Near the end of the rapid collapse stage ( $\mathrm{t}=1.20$ days) the concentration profile has a shape similar to that of the shorter lived gels at a similar point in their collapse, e.g. $t=0.25$ days in the 3 hour gel. Below the sharp interface with the supernatant there is a layer about $20 \mathrm{~mm}$ thick whose concentration increases from zero to $20 \%$ as height decreases. Below this is a thin layer with homogeneous concentration of $20 \%$. The interface between these two layers looks fairly sharp. The constant concentration layer blends smoothly into a layer of dense sediment which continues to increase in concentration as height decreases.

The layer of dense sediment appears non-uniform in density. Large oscillatory fluctuations are observed throughout the dense sediment. The largest of these "bumps" in 
the concentration occurs at the very bottom where the concentration appears $2 \%-3 \%$ lower than it is $5 \mathrm{~mm}$ higher up. These features become less pronounced as the sediment compacts.

Around 6 hours after the delay time ( $\mathrm{t}=1.25$ days) a sharp interface has formed between the dense sediment layer and the less dense layer on top. The less dense layer continues to settle on top of the sediment layer.

As in the shorter lived gels, once the rapid collapse stage is over, the sediment gradually compacts under its own weight, i.e. concentration increases while the interface between the sediment and supernatant decreases in height. The concentration of the sediment is mainly uniform but we still observe some small amplitude bumps in the profile.

\subsubsection{Creeping Sedimentation}

Figure 5.4 shows the concentration profiles for a sample exhibiting creeping sedimentation behaviour. In order to obtain creeping behaviour we have studied the previous sample ( $\tau=25$ hours) but filled the sample cell to height of $55 \mathrm{~mm}^{\dagger}$.

Initially the suspension has constant, homogeneous concentration $(\phi \approx 20 \%)$. During the first twelve hours only the top and bottom few millimetres increase in concentration slightly while the bulk of the suspension remains at the original concentration. After about a day, a sharp interface is clearly observed at the top of the settling suspension separating it from the clear supernatant left on top.

Over the next 30 days the suspension continues to sediment slowly. A gradient in concentration begins to form at the bottom of the suspension. This denser sediment merges into a region of constant concentration above it in a smooth manner. As the boundary settles the whole suspension becomes gradually more concentrated while maintaining the shape of the profile, i.e. the whole suspension is becoming more concentrated at the same rate. The layer of concentration gradient grows in thickness until it almost fills the whole of the sample after 31 days.

\footnotetext{
${ }^{\dagger}$ The origin of height-dependent settling properties will be discussed in more detail in chapter 6
} 
Some small oscillatory fluctuations begin to form in the middle of the sediment after 2 days. These grow a little in amplitude and move downwards in the suspension over the next 12 days but have disappeared after 25 days of settling. Even after 1 month we note that this sample has not yet finished settling nor has it shown any evidence that it may undergo a rapid collapse.

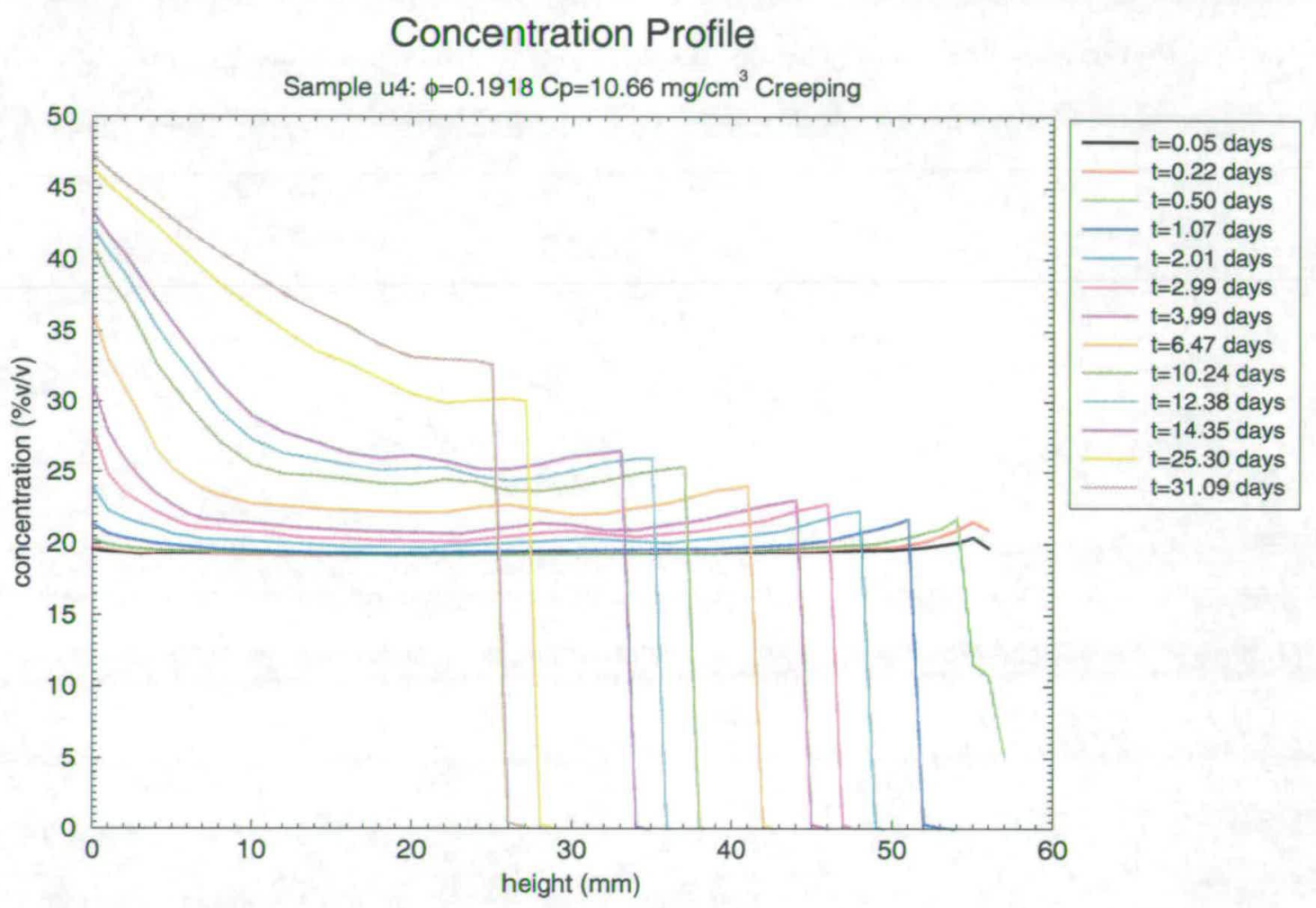

Figure 5.4: Concentration profiles of a gel exhibiting creeping sedimentation. Each line represents the concentration profile measured at different times (black $=0.05$ days, red $=0.22$ days, light green $=0.50$ days, dark blue $=1.07$ days, light blue $=2.01$ days, magenta $=2.99$ days, light purple $=3.99$ days, orange $=6.47$ days, dark green $=10.24$ days, turquoise $=12.38$ days, dark purple $=14.35$ days, yellow $=25.30$ days, and brown $=31.09$ days).

\subsection{Interpretation of Concentration Profiles}

Using ultrasonic density profiling we have been able to identify the difference between the two settling behaviours previously observed by dark-field viewing. We now discuss the concentration profiles in the context of what is observed in dark-field imaging. 


\subsubsection{Delayed Sedimentation}

The same features are observed in all gels exhibiting delayed sedimentation irrespective of the length of the delay. The main difference between short and long lived gels is that the concentration features displayed by longer lived gels are much more pronounced than those in the short $\tau$ gels. For example, small rises at the top and bottom of the suspension formed during the delay become much more pronounced as the lifetime of the gel increases. This may just be due to there being more time for variations in concentration to grow in long delay time gels than in the shorter lived samples.

\section{Delay Stage}

The concentration of a transient gel remains constant throughout almost the whole of its height during the delay stage. It is known from direct observation that local breaking of the gel occurs due to channels which begin to form early on. Breaking of the gel structure produces more compact clusters and thus a rise in concentration. However, this technique for measuring concentration is insensitive to such small variations as channels as it can only detect the average concentration of the suspension at any height. Channels are thin $(1 \mathrm{~mm})$ when compared with the width of the suspension which the ultrasonic pulse must travel through $(10 \mathrm{~mm})$. Unless the structure of the broken gel fragments is very compact, channels will not be detected as the rise in concentration they create will be too small to affect the average concentration of the bulk.

Regions of slightly raised concentration correspond to large areas identified by darkfield observation to be made up of broken up gel. Small rises in concentration detected at the top of the suspension correspond to the formation of the bright patches which eventually form streamers seen in figure 4.6. Similarly, small rises in concentration at the bottom of the suspension during the delay time correspond to the formation of the bright bands, figure 4.13. These features can be detected as they span the whole width of the sample. 


\section{Rapid Collapse}

The concentration of the clear supernatant left behind by the settling suspension is zero, i.e. no colloidal particles are left behind when the gel begins to collapse. As observed in dark-field and other imaging techniques, the interface between the settling particles and the supernatant is sharp. The concentration profiles show a slight rounding of the interface indicating that some material lags behind the main suspension. This is also detected in dark-field where the interface becomes blurred during rapid collapse.

One obvious feature of the concentration profiles during this time is the persistence of a layer which maintains the original concentration of $20 \%$ for a long time into the rapid collapse stage. From dark-field observations we know that material in this region is circulating around the sample cell. In short delay time gels this circulation of material can be very rapid, breaking the gelled structure into large clusters which sediment much faster than the individual particles. During this process clusters break and reform as they move around and collide with each other. We can therefore think of the sample as being a suspension of clusters which are well mixed by circulation. The concentration of this suspension will be unchanged from that of the gel structure before breaking. This region of constant concentration should persist so long as the suspension of clusters remains well mixed by circulation.

After some time a gradient in concentration begins to form underneath the top interface. Dark-field observations show that large scale circulation of material is no longer present in this region which is visible to the eye as a layer of less dense fluid-like suspension.

A possible explanation of this gradient may be that once mixing of the suspension by circulation has slowed down the clusters can settle at different speeds depending on their size, local density and structure. Thus, so long as the concentration of clusters is not so high as to prevent settling completely, the clusters will segregate according to their settling velocity and a concentration gradient will form.

This segregation is illustrated in figure 5.5 (solid lines) which shows the height variation with time of contours of specific concentration. This figure displays the same data as presented in the concentration profile in figure 5.3 but plotted as the height of a particular concentration against time. Once the suspension begins to settle rapidly the 
contours separate from each other indicating that each one is settling at a different rate. The contours accelerate up to a time of 1.25 days where the higher concentration contours suddenly slow down as they come into contact with the dense sediment that has formed at the bottom of the sample cell.

\section{Contours of Constant Concentration}

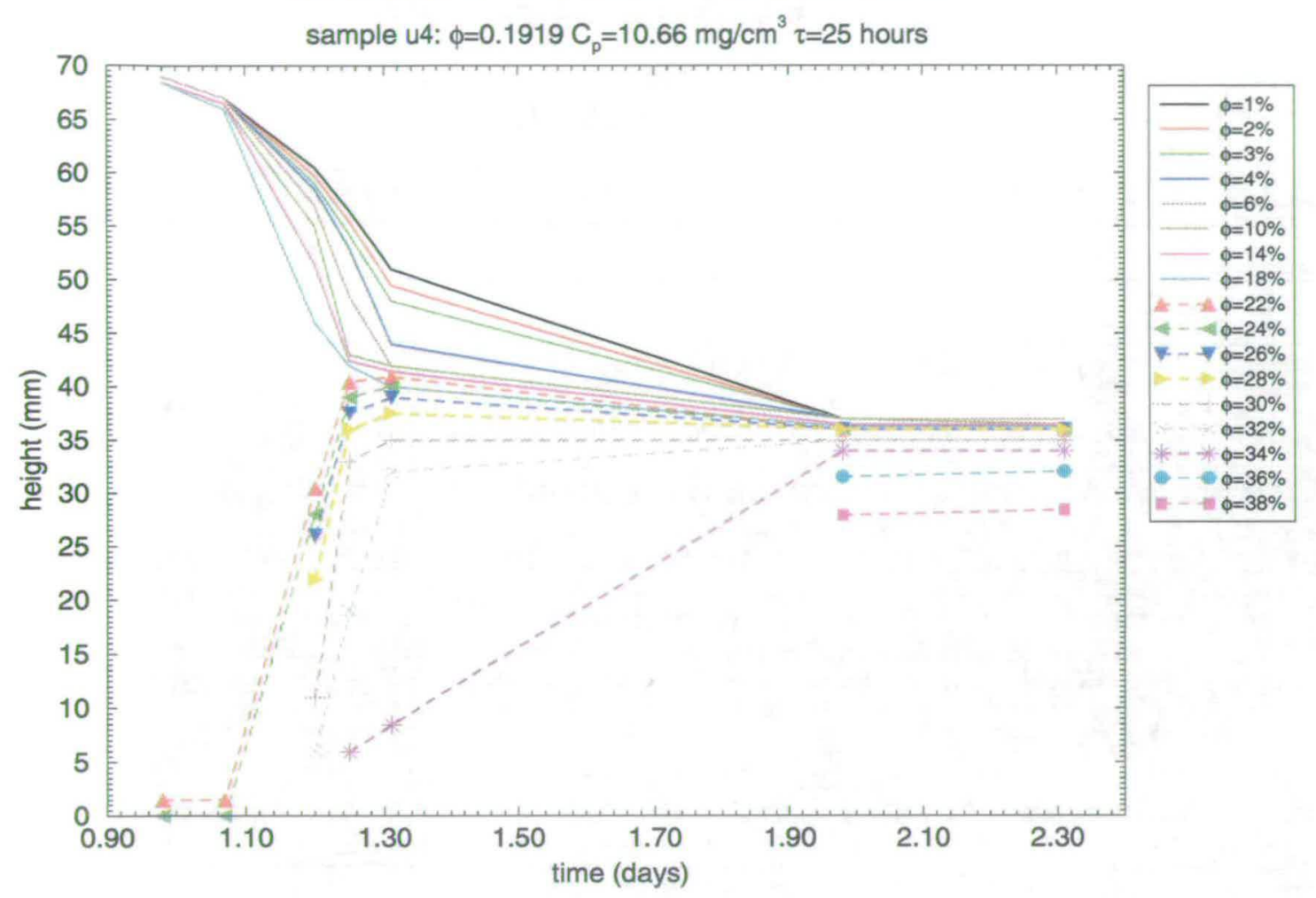

Figure 5.5: Plot of contours of constant concentration height against time for a gel exhibiting delayed sedimentation with delay time of 25 hours. Each line represents a different concentration. Solid lines indicate contours of concentration less than or equal to $20 \%$ and dashed lines indicate contours of concentration greater than $20 \%$

The dynamics of a concentrated suspension of clusters are complex and we cannot assume that the clusters settle independently of each other or that the suspension behaves in a similar way to a suspension of polydispersed particles. We must consider the hydrodynamics of the system in order to create a realistic model. Thus at this stage we can only speculate on the mechanism of concentration gradient formation.

The concentration profiles also show the formation of a dense sediment at the bottom of the cell which grows in thickness as the suspension of clusters settles. This sediment 
corresponds to a region of slower moving fluctuations observed in dark-field which builds up from the bottom. The boundary between the dense sediment and the upper part of the collapsing suspension is very diffuse in dark-field. This is confirmed by the smooth variation in concentration observed with ultrasound in this region. Contours of constant concentration in the sediment are plotted as dashed lines in figure 5.5. The top of the dense sediment as indicated by the $\phi=22 \%$ contour grows in height until it encounters the most concentrated contour in the settling suspension $\phi=18 \%$.

The oscillatory variations of concentration observed in the sediment as it forms suggest that the packing of colloidal material is non-uniform. Reduction in concentration may be caused by the presence of large voids where little colloidal material is present. However, why these may form is unclear.

\section{Compaction}

The final stage of gel collapse is the compaction of the amorphous sediment. The concentration profiles show the sediment becoming more concentrated in a uniform way as it compresses. The sediment is formed by deposition of clusters. Thus, when first formed the sediment will have a partly fractal structure. With time the particles in the sediment will slowly rearrange due to thermal fluctuations and compaction and so the concentration of the sediment will increase.

\subsubsection{Creeping Sedimentation}

It is clear from the concentration profiles and dark-field observations that creeping behaviour is very different from delayed sedimentation. Samples exhibiting creeping behaviour compress more or less uniformly from the very beginning of the settling process. This is illustrated in figure 5.6 which plots the height of contours of constant concentration with time for a sample exhibiting creeping sedimentation behaviour.

It is clear from figure 5.6 that all the contours settle in more or less the same way indicating that the suspension is compressing uniformly. However, the concentration profile, figure 5.4 shows that a dense sediment forms at the bottom of the sample and grows while the bulk of the suspension maintains a homogeneous concentration. 
U4h - creeper - $9 \mathrm{mgml}$

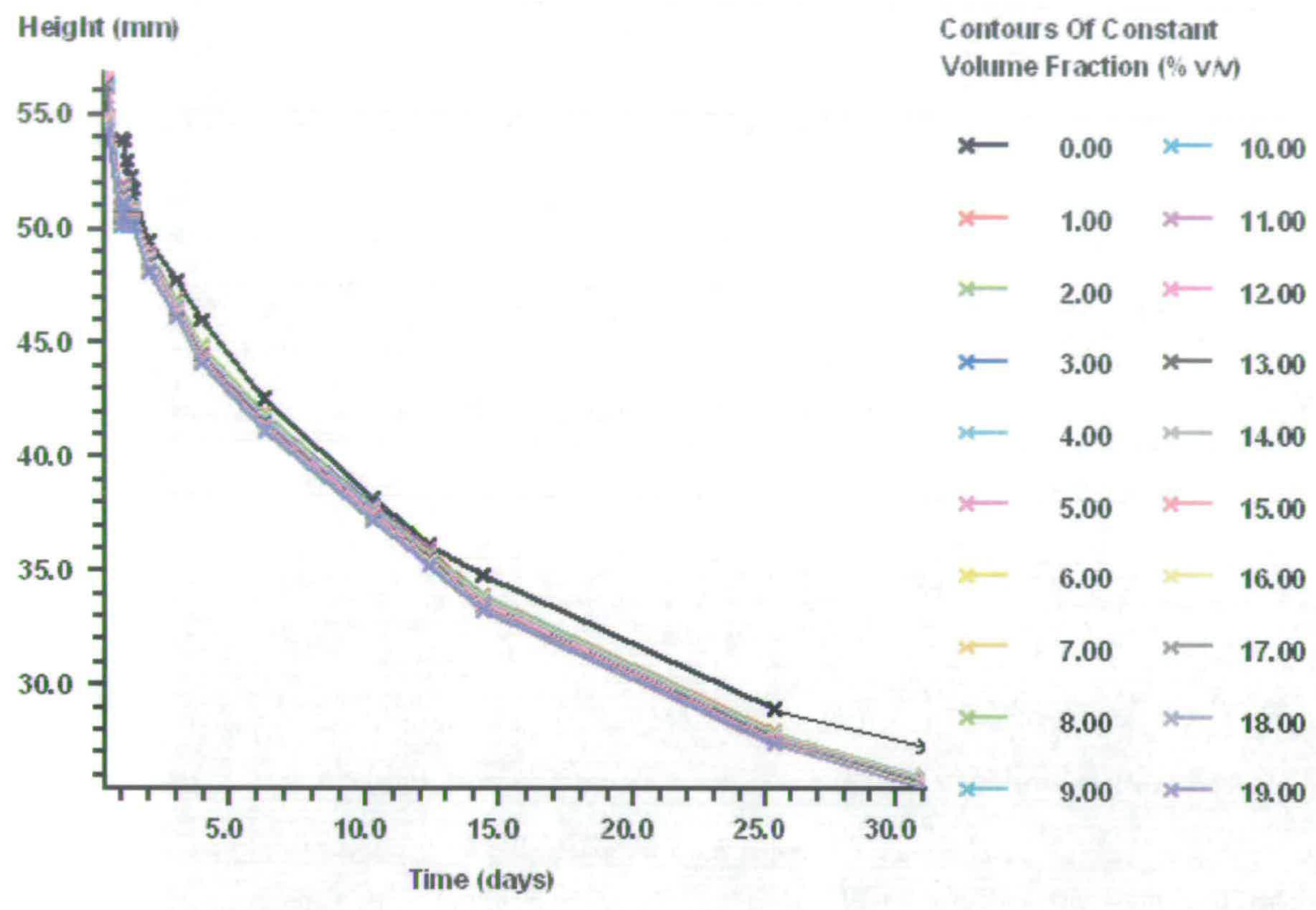

Figure 5.6: Plot of contours of constant concentration with time for a sample exhibiting creeping sedimentation. Each line represents a different contour of specific concentration.

In this case the bottom of the sample is being crushed from above by the bulk of the suspension which is settling as a single entity. As the suspension settles, the material at the bottom is compressed and forms a region of more compact structure where the sediment is more concentrated. As settling proceeds so material above the bottom layer becomes more compact while the material below becomes even more concentrated. This continues until the whole suspension is made up of the compacted sediment whose concentration decreases with increasing height.

The region of slightly raised concentration near the top of the sample corresponds to where we find a bright coarsened texture in dark-field observations, see figure 4.8. This begins to form from the very beginning of the settling as is observed in the dark-field images. However, the region of raised concentration does not appear to propagate downwards into the suspension in the way that the coarse texture in dark-field does. 


\section{Chapter 6}

\section{Size Effects}

\subsection{Introduction}

The delay time of a particular gel does not only depend upon its composition. Factors such as sample size, geometry, shear history and temperature can all affect the length of the delay time measured. In this section we shall concentrate on the effect of varying the height and width of a gel upon its delay time.

\subsection{Strong and Weak Gels}

In his thesis Meeker made a study of the dependency of delay time on the size and shape of the sample [16]. He found that gels with high polymer concentrations showed long delay times which increased as the height of the sample was reduced. For gels with delay times of less than ten hours he found that $\tau$ was independent of initial sample height. From these observations transient gels were categorised into two regimes known as strong and weak. Strong gels had stronger depletion attractions and showed heightdependent delay times while weak gels had weaker interactions and showed heightindependent delay times.

This categorisation of the gels into strong and weak was based upon observations made in just three samples which were restricted in their maximum height by the dimensions of the sample cell, $4 \mathrm{~cm}$ in height. It was suggested that perhaps all gels would show 
height-independent delay times if only they could be made tall enough.

The width dependency of gel lifetimes was also investigated by Meeker for weak gels by simply turning a tall and thin sample on its side to make a short and fat sample. There was no effect on the delay time. This experiment was not repeated for a strong gel.

\subsection{Height Dependency: A More Detailed Study}

Height-dependent and height-independent delay times have been observed in this study. Figure 6.1 shows a plot of delay time against initial sample height for a gel of composition $\phi=0.20, C_{p}=7.01 \mathrm{mgcm}^{-3}$ as measured in a cylindrical cell of diameter $\approx 1 \mathrm{~cm}$. This sample clearly has a height-dependent delay time for all initial heights below 88 $\mathrm{mm}$.

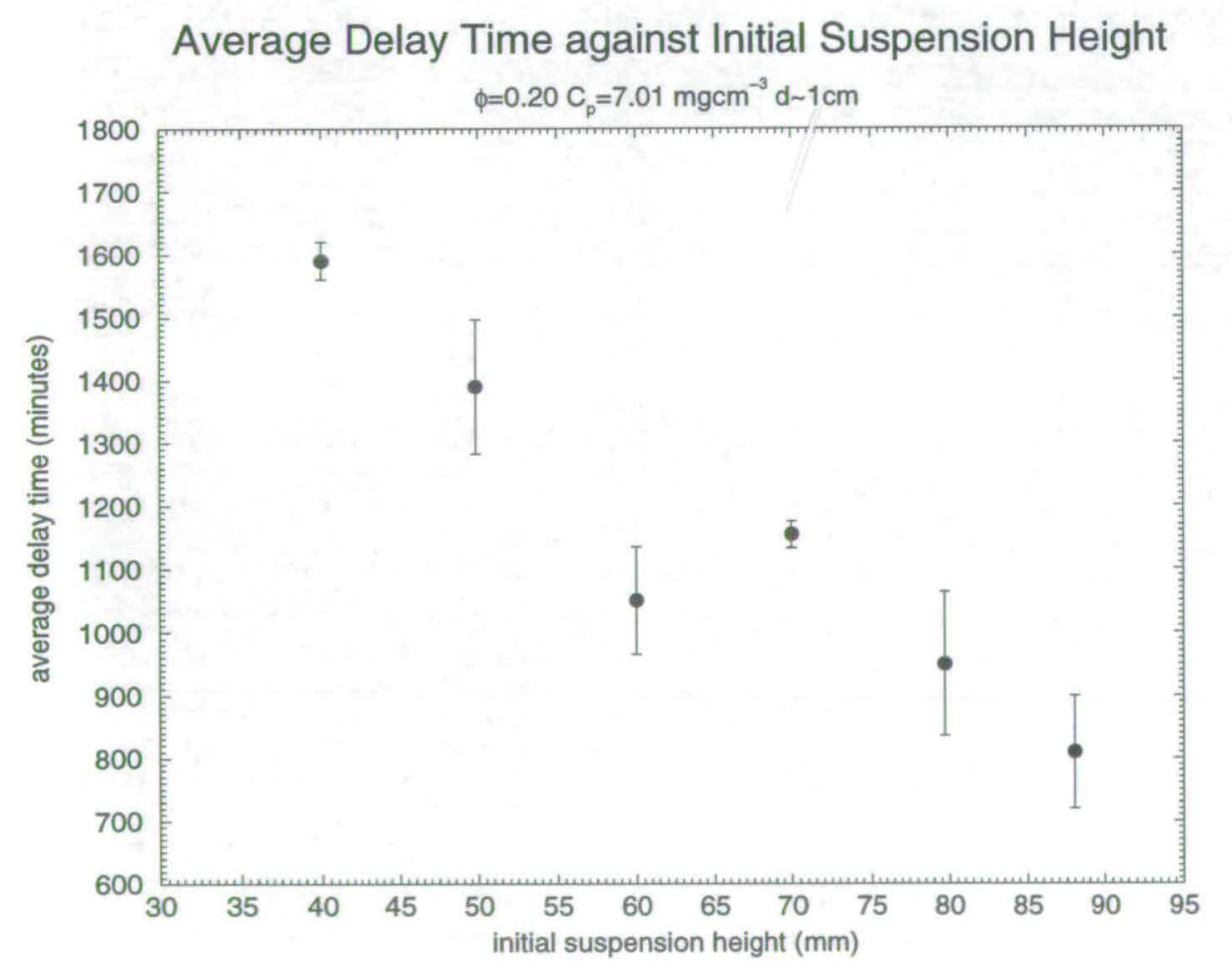

Figure 6.1: Plot of delay time against initial sample height showing height dependency of the delay time for a gel of composition $\phi=0.20, C_{p}=7.01 \mathrm{mgcm}^{-3}$ in a $1 \mathrm{~cm}$ diameter cylindrical cell. 
The fast (rapid collapse) settling velocity $u_{f}$ decreases as the sample gets shorter as shown in figure 6.2. The slow settling velocity remains unchanged as the height is reduced within the range of height studied. This particular sample has not been made tall enough to show clear height-independent delay times.

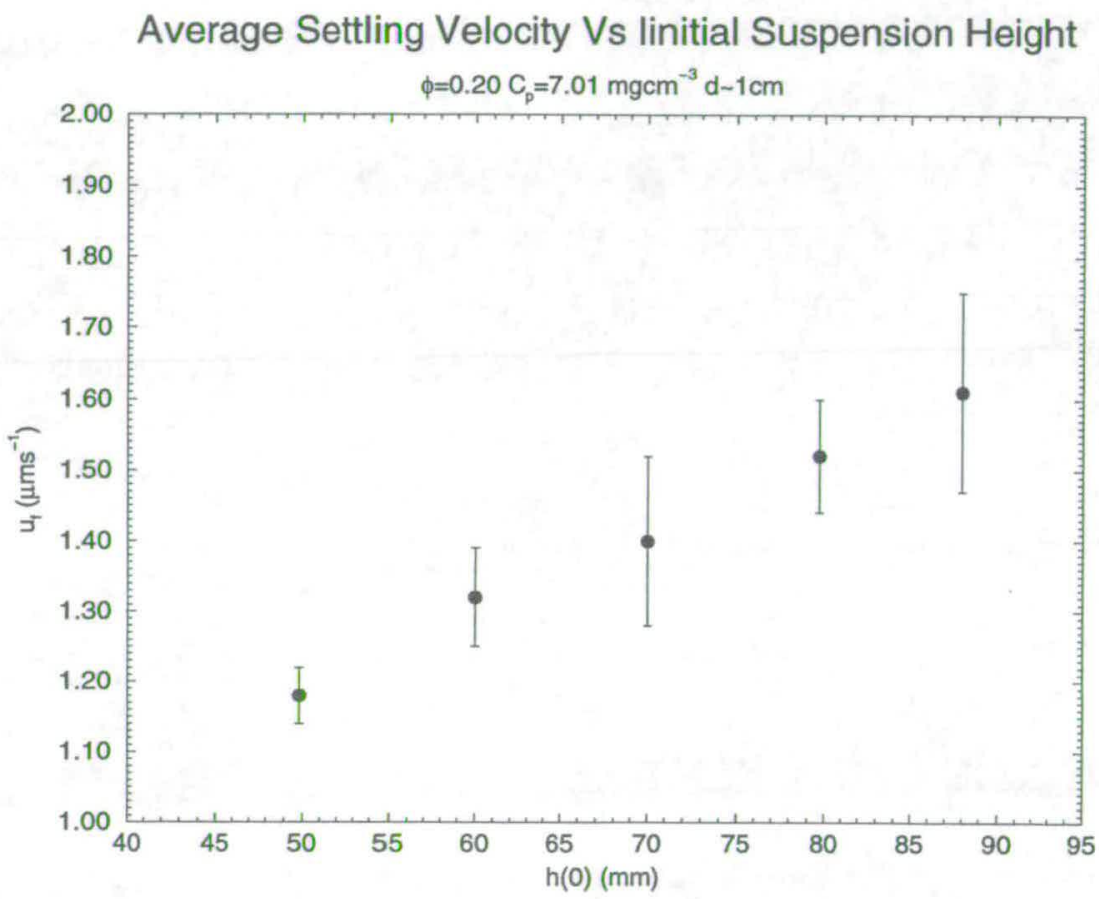

Figure 6.2: Plot of fast settling velocity against initial height for the same sample as in the previous figure.

\subsubsection{Creeping Sedimentation}

Creeping sedimentation behaviour can be induced in a gel sample if the initial height is made small enough. This is illustrated in figure 6.3 which shows the sedimentation profiles of two samples with $\phi=0.20$ and $C_{p} \approx 7 \mathrm{mgcm}^{-3}$ but different initial height (35 $\mathrm{mm}$ and $15 \mathrm{~mm}$ ). The profiles have been shifted in the height axis in order to produce a common origin. The taller sample shows delayed sedimentation behaviour with a delay time of around twelve hours whereas the shorter sample displays creeping behaviour. These measurements were made in the standard square cells described in section 4.3.1. 


\section{Shifted Sediment Height against Time}

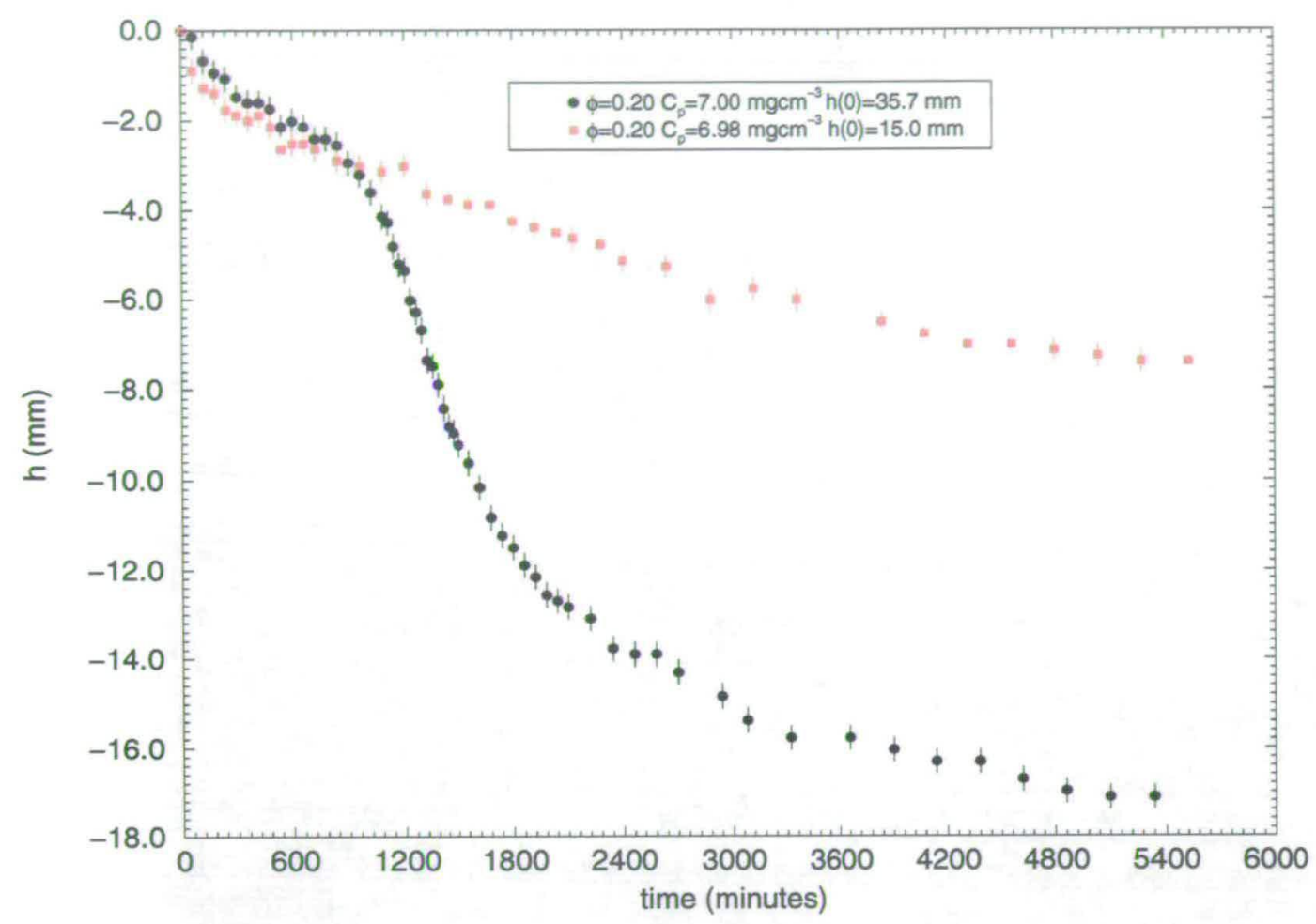

Figure 6.3: Sedimentation profiles of two samples with $\phi=0.20$ and $C_{p} \approx 7 \mathrm{mgcm}^{-3}$ but of different heights (circles: $\mathrm{h}(0)=35.7 \mathrm{~mm}$, squares: $\mathrm{h}(0)=15.0 \mathrm{~mm}$ ). The taller sample shows a delay time of around 12 hours whereas the shorter sample exhibits creeping sedimentation behaviour.

\section{Erratic Behaviour}

Near the crossover from delayed sedimentation to creeping sedimentation we observe what may be called erratic behaviour. In this case, a sample may change its settling behaviour from delayed sedimentation to creeping from experiment to experiment as shown in figure 6.4. The gel shown in this example is the same one as shown in the images in figure 4.6 (a)-(l) but the initial height is now only $1 \mathrm{~cm}$. The sample is still in the original square cell. On two occasions this sample showed delayed sedimentation with a delay time of around seven hours. On two other occasions the sample showed creeping sedimentation behaviour. 


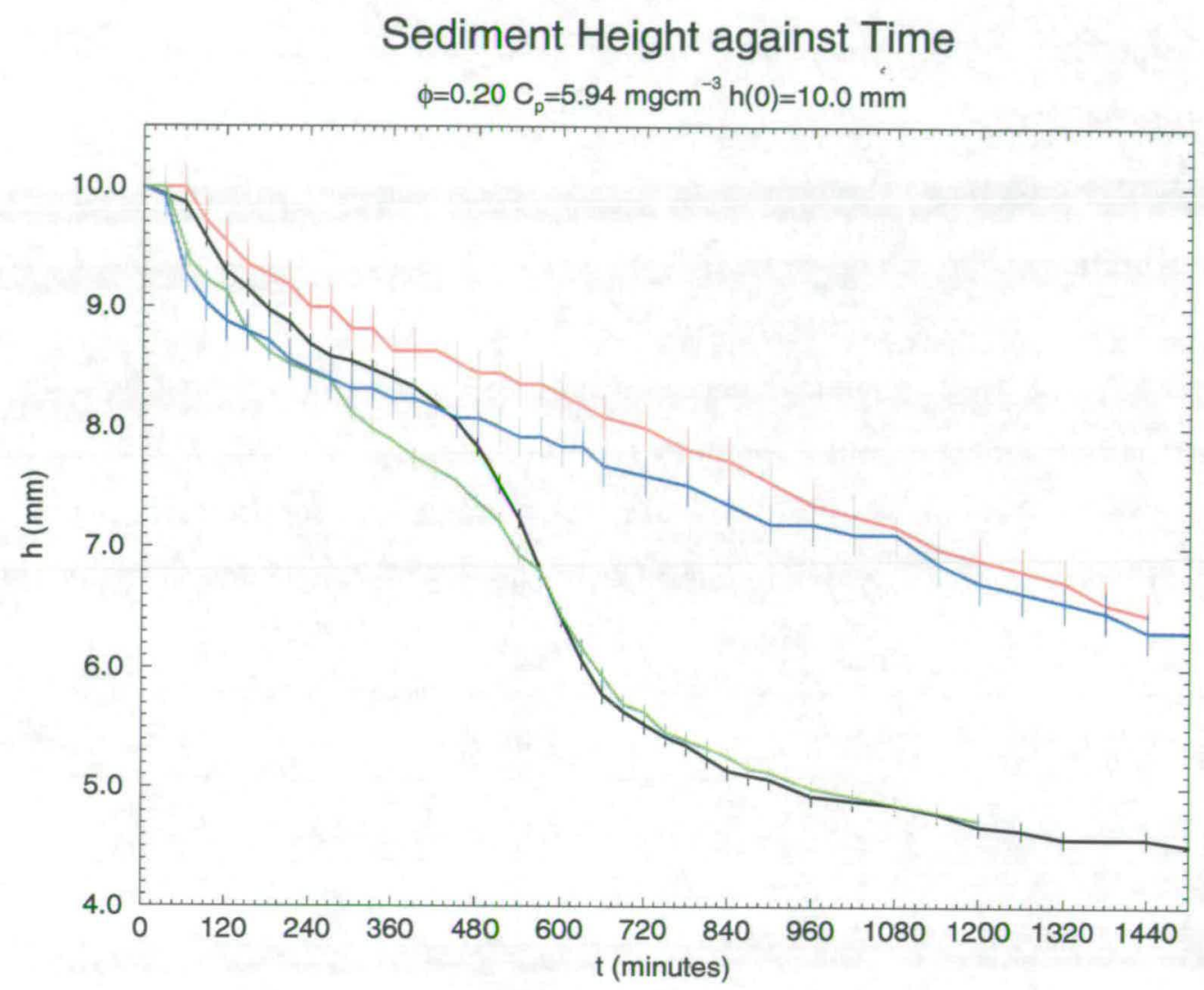

Figure 6.4: Sedimentation profiles of a gelled sample showing erratic settling behaviour. Each line represents a different measurement of the settling behaviour carried out under the same external conditions.

\subsection{Height and Width Dependency}

We now present a more detailed study of the way in which sample height and width determine the delay time of a particular gel.

\subsubsection{Experimental Method}

The model system used was as described in section 4.2 but on this occasion the particles and polymer are suspended in pure cis-decalin. The sample under study had volume fraction $\phi=0.20$ and polymer concentration $C_{p}=7.14 \mathrm{mgcm}^{-3}$. In a standard sample geometry we would expect this sample to show creeping behaviour. 
A set of cylindrical glass cells with different diameters were specially made for this study in order to eliminate any differences in boundary conditions due to the cell construction. The settling behaviour of the gel was recorded by time-lapsed video under normal lighting conditions, i.e. not dark-field. Each experiment was repeated at least three times.

\subsubsection{Results}

The average delay time in minutes and its standard deviation is tabulated in table 6.1 against the initial height $h(0)$ and width $w(0)$ of the gelled sample. The delay time measured in the thinest cell $(w(0)=5 \mathrm{~mm})$ was found to be consistently lower than expected. This is most likely due to improper mixing of the sample due to the thinness of the cell.

\begin{tabular}{|c|c|c|c|c|c|c|c|}
\hline \multirow{2}{*}{$\begin{array}{c}h(0) \\
(\mathrm{mm})\end{array}$} & \multicolumn{7}{|c|}{$w(0)(\mathrm{mm})$} \\
\cline { 2 - 8 } & 5 & 7 & 10 & 15 & 20 & 25 & 30 \\
\hline 5 & - & - & - & - & - & - & $300 \pm 42$ \\
\hline 7 & - & - & - & - & - & - & $185 \pm 35$ \\
\hline 10 & $270 \pm 30$ & CREEP & $415 \pm 333$ & $243 \pm 173$ & $185 \pm 64$ & $200 \pm 44$ & $188 \pm 35$ \\
\hline 15 & - & - & - & - & - & - & $167 \pm 55$ \\
\hline 20 & - & $770 \pm 75$ & $350 \pm 221$ & $197 \pm 67$ & $170 \pm 46$ & - & $183 \pm 25$ \\
\hline 25 & - & - & - & $160 \pm 30$ & - & - & $180 \pm 60$ \\
\hline 30 & $273 \pm 6$ & $473 \pm 8$ & $495 \pm 84$ & - & - & - & - \\
\hline 35 & - & - & - & - & - & - & $157 \pm 15$ \\
\hline 70 & - & $345 \pm 21$ & - & - & - & - & - \\
\hline 75 & - & - & $485 \pm 7$ & - & - & - & - \\
\hline 80 & $365 \pm 35$ & - & - & - & - & - & - \\
\hline
\end{tabular}

Table 6.1: Average delay time $\bar{\tau}$ in minutes as a function of initial sample height $h(0)$ and width $w(0)$. 


\section{Crossover Width}

When the initial height is $20 \mathrm{~mm}$, we find a width independent delay time above an initial width of $10 \mathrm{~mm}$. This is shown in figure 6.5 which shows the delay time against sample width for three separate measurements. Above $10 \mathrm{~mm}$ width the average delay time of the gel is about 180 minutes which suddenly increases to 350 minutes at a width of $10 \mathrm{~mm}$. Below $10 \mathrm{~mm}$ the delay time then increases further to 770 minutes at a width of $7 \mathrm{~mm}$.

These observations suggest that for a sufficiently tall sample there exists a crossover width $w_{x o}$ below which the delay time is dependent on the width of the sample. For this sample $w_{x o} \approx 10 \mathrm{~mm}$.

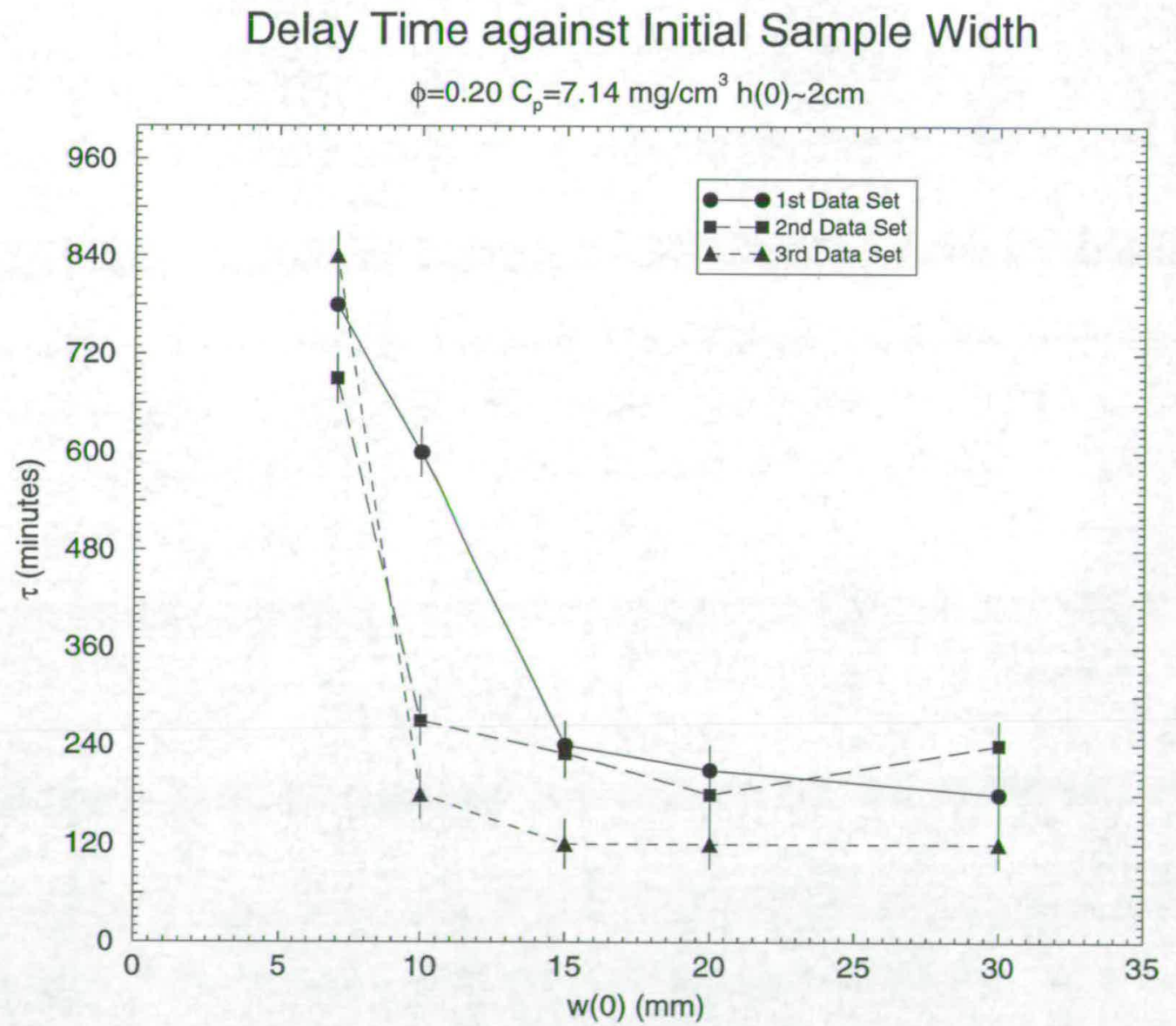

Figure 6.5: Plot of delay time $\tau$ against initial sample width $w(0)$. The initial sample height $h(0)$ was approximately $20 \mathrm{~mm}$. 


\section{Crossover Height}

When the suspension has a width well above $10 \mathrm{~mm}$ we find that the delay time is height-independent ( $\tau \approx 180$ minutes) down to a height of $5 \mathrm{~mm}$ where it suddenly increases to 300 minutes, see figure 6.6. It was not possible to study heights lower than this due to large meniscus effects. This suggests that for sufficiently wide samples $\left(w(0)>w_{x o}\right)$ there exists a crossover height $h_{x o}$ below which the delay time becomes dependent upon sample height. For this sample $h_{x o} \approx 5 \mathrm{~mm}$.

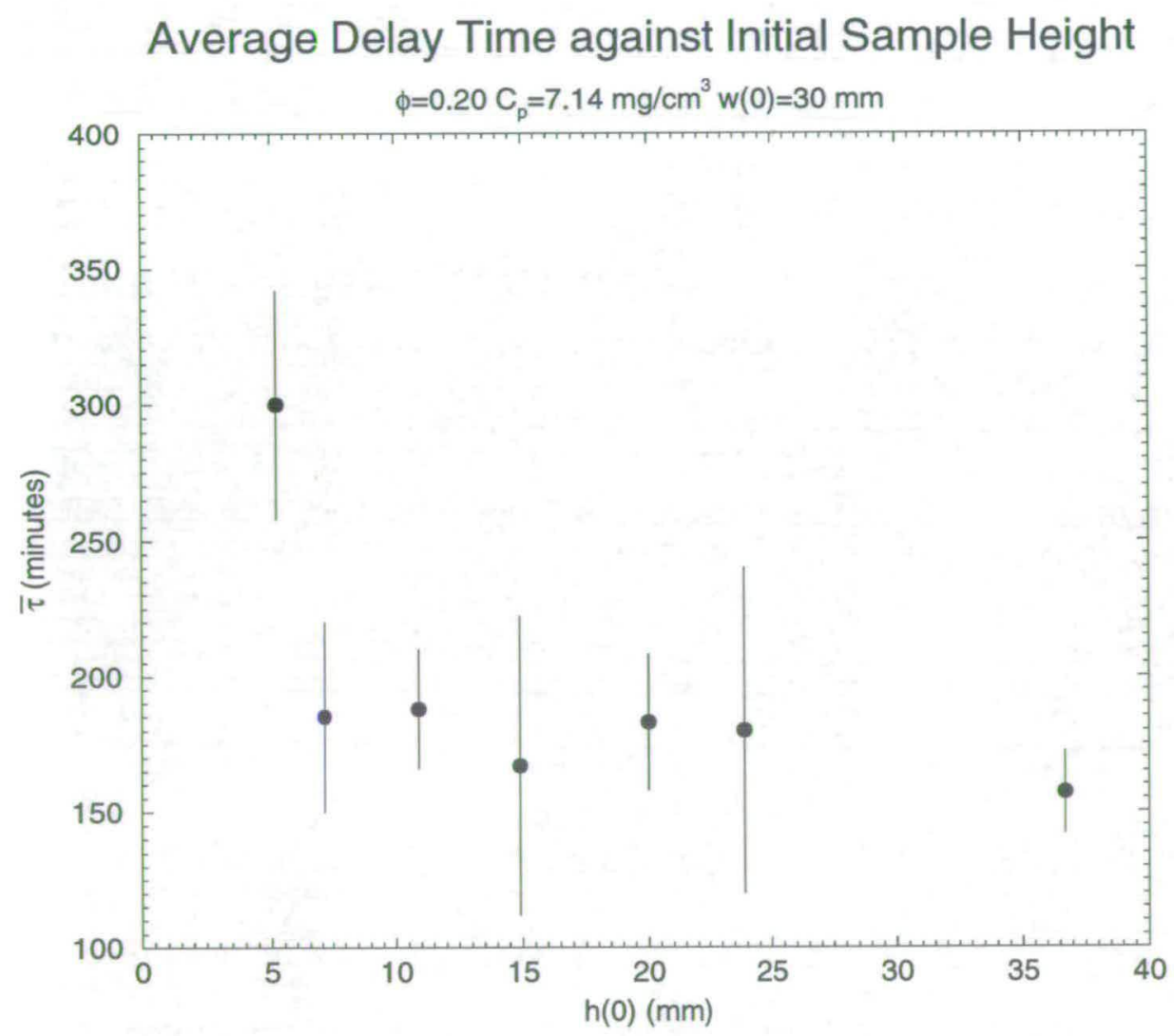

Figure 6.6: Plot of average delay time against initial sample height in a sample much wider than the crossover width.

Height-dependent delay times are found at heights above $5 \mathrm{~mm}$ when the sample width is below the crossover value. The $7 \mathrm{~mm}$ wide sample begins to show height dependent delay times at a height below $30 \mathrm{~mm}$, see figure 6.7. This sample also exhibits creeping sedimentation behaviour once it reaches a height of $10 \mathrm{~mm}$. 


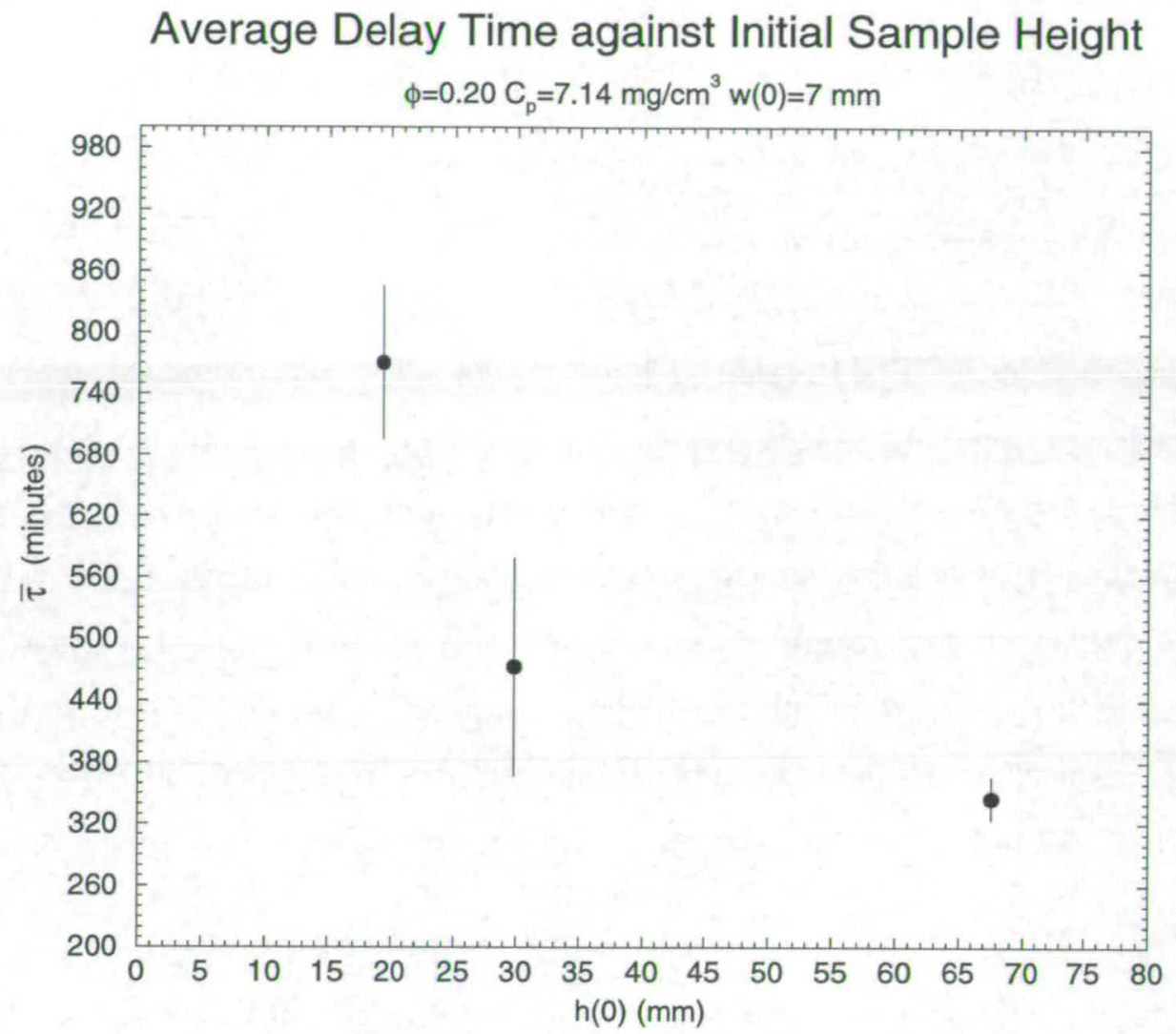

Figure 6.7: Plot of average delay time against initial sample height in a sample narrower than the crossover width.

\subsubsection{Some Observations}

The height and width of a gelled suspension can affect the delay time measured and even the type of settling behaviour observed. From the data presented we see that there are regions in sample height and width where the delay time is independent of the dimensions and other regions where the size of the gel determines the delay time measured. Thus the idea of a gel being simply size-dependent (strong) or sizeindependent (weak) is not strictly valid. Any gel can exhibit either behaviour if its dimensions are chosen appropriately.

The measured delay time is found to be highly irreproducible near the crossover width $w_{x o}$ when the sample is about $2 \times w_{x o}$ in height. Far from this region, the delay time is much more reproducible, see table 6.1. This is another example of erratic behaviour as seen at the crossover between delayed sedimentation and creeping behaviour. 
The crossover height is about half the size of the crossover width. This suggests that there is one crossover length-scale which for this sample is around $5 \mathrm{~mm}$.

\subsubsection{A Theoretical Explanation}

Following the approach of Potanin and co-workers [42, 43], a recent theory by Evans [44] attempts to predict the length-scale at which the delay time of a gel becomes sizedependent. The theory postulates that the observed characteristic crossover length is the distance over which mechanical stresses can be transmitted through the gel. Thus, if the initial height of the sample is shorter than this length-scale then the proximity of the bottom of the container can provide extra support to the gel. A similar argument applies for the effect of width, i.e thin samples are able to support themselves from the walls. Here we present a simple argument to illustrate the origins of this mechanical length-scale.

Figure 6.8 shows the forces acting on a thin slice of a gelled suspension as it settles under its own weight at velocity $u$. There are two mechanisms by which the gel can resist settling. Firstly, as the gel settles solvent must flow upwards through the fractal structure in order to conserve mass. This will create an upwards force on the gel so long as the solvent flows. This force is labelled as drag in figure 6.8.

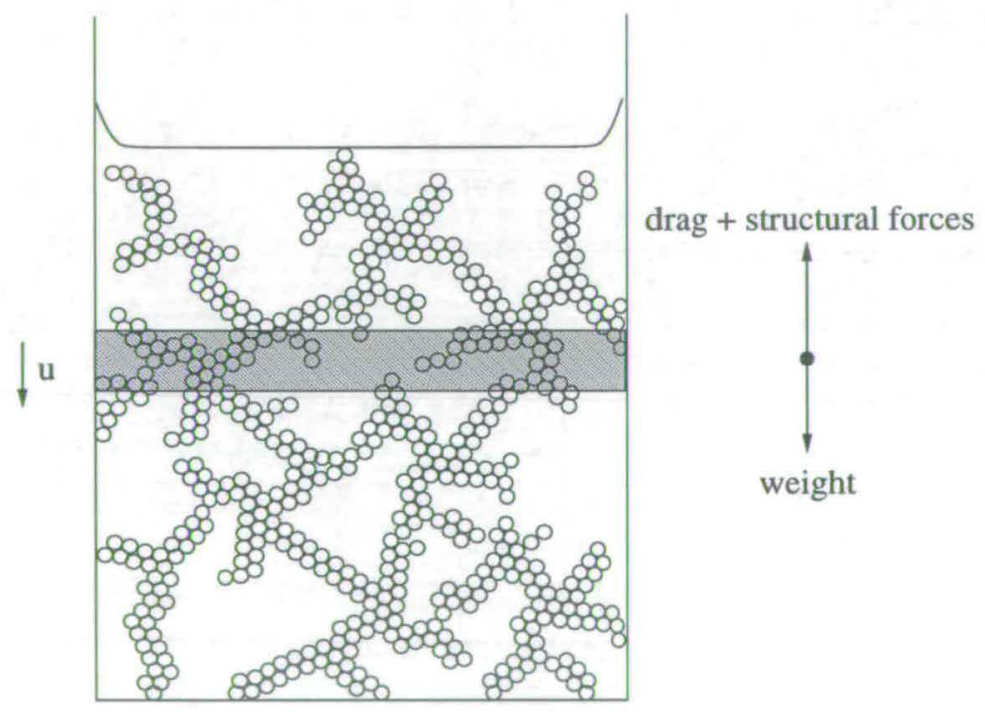

Figure 6.8: Diagram showing the balance of forces on a thin slice of a gelled suspension settling at velocity $u$. 
The gel can also be supported by its own structure. Based on a $2 \mathrm{D}$ simulation study of reversible DLCA [19], we picture a transient gel as an interconnected network of particle chains where each chain is two or more particles in diameter, see figure 2.7 (B). As these chains compress they can transfer stresses through the structure to support it. Haw's simulations [19] show that the chains are transient in nature and have a finite probability of breaking due to thermal rearrangements. Under the influence of gravity, this thermally activated breaking may be enhanced by stresses on the structure.

Support due to back flow of solvent will be present in samples of all sizes and, in the absence of structural support, will lead to the suspension settling at its terminal velocity. Structural support will only be present if the gel structure can transmit stresses from the walls and base to the bulk of the dispersion.

For simplicity let us consider the origin of height-dependency alone. As the gel settles under its own weight, stresses are transmitted through the network of elastic chains. Each chain has a finite lifetime. The force transmitted through an element of the gel due to chain compression $F$ is assumed to be proportional to the rate of compression. The rate of compression is proportional to the gradient of the settling velocity $u$ which is greatest at a height where settling material meets the static sediment formed at the bottom of the sample, see figure 6.9. This level defines the base of a region of significant compression which extends to a height $Z_{\text {cross }}$ where $F \sim d u / d z \rightarrow 0$. The value of $Z_{\text {cross }}$ depends on the strength, lifetime, and sedimentation rate of the network of chains.

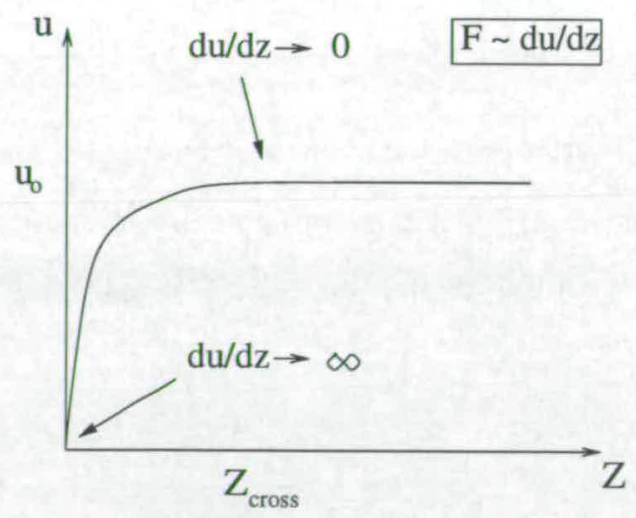

Figure 6.9: Schematic diagram of the variation of settling velocity with height in a network of locally elastic chains. The force transmitted through the chains is proportional to the gradient of velocity $F \sim d u / d z . u_{o}$ is the terminal settling velocity of the suspension. 
If the sample is shorter than the height of the compression region $\left(h(0)<Z_{\text {cross }}\right)$ then the structure will always be able to transmit stresses to the whole of the suspension and the gel will be height-dependent. Conversely, if $h(0)>Z_{\text {cross }}$ then the compression region does not extend to the top of the sample. Thus the upper region of the gel is not supported by the structure below. In this case only back-flow will support the upper part of the structure which will settle at its terminal velocity. All properties of the top of the suspension, including its delay time, must therefore be independent of height.

From dimensional analysis the crossover length-scale is given by

$$
Z_{\text {cross }}=\sqrt{\frac{\eta}{\gamma}}
$$

where $\eta$ is the bulk viscosity of the gel and $\gamma$ is the coefficient of resistance to solvent flow through the structure. The bulk viscosity of the gel arises due to the ability of the chains to store elastic energy as they are compressed. This energy is dissipated when chains are broken due to thermal rearrangement.

Equation 6.1 suggests that the crossover length for a strong gel, i.e. large $\eta$, is larger than for a weak gel. This explains why height-dependent delay times have previously only been found in gels with high polymer content [16]. The strong gels are likely to have crossover heights larger than the sample cell thus they will always be in the height-dependent regime. In contrast, weak gels will have crossover heights which are much shorter than the typical sample size so that they will always be in the heightindependent regime.

A similar argument [44] predicts a crossover length-scale in the width direction. In this case the bulk viscosity in equation 6.1 is replaced by a shear viscosity and we are now interested in knowing if the bulk of the gel can be supported by the walls.

\section{Predictions}

Whether a gel exhibits a size-dependent delay time depends upon its initial height and width in relation to the crossover height $h_{x_{0}}=Z_{\text {cross }}$ and width $w_{x o} \approx 2 \times Z_{\text {cross }}$. Figure 6.10 summarises the four expected regimes. 


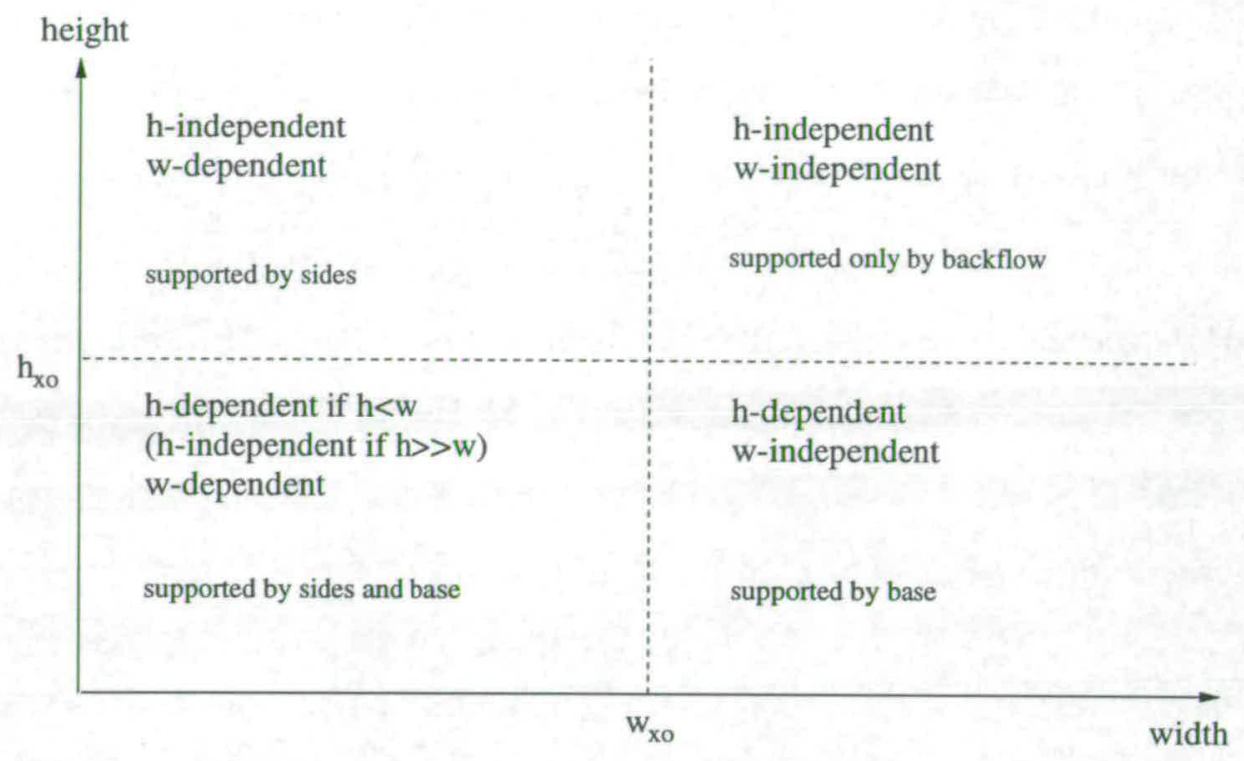

Figure 6.10: Schematic diagram showing the expected height and width behaviour of a transient gel.

When the height and the width of the suspension are larger than the crossover height and width then the gel is unable to support itself through its own structure. Only the back flow of solvent will resist the collapse and the suspension will settle at its terminal velocity. In this regime we expect to find a delay time which is independent of the sample size.

For a sample which is below the crossover height but wider than the crossover width we expect to find a delay time which depends on height but not upon width. In this case the gel is able to support itself by the transmissions of stresses from the base while remaining unaware of side wall effects. We expect the delay times measured in this regime to be longer than those measured in the size-independent regime since there is now some extra support from the gel structure.

A similar situation occurs when the sample is taller than $h_{x o}$ but narrower than $w_{x o}$. In this case the structure can support itself by the side walls and thus will have a width dependent delay time. Again we expect to find longer delay times in this regime than in the size-independent regime.

Finally when the sample is shorter than $h_{x o}$ and narrower than $w_{x o}$ we should find a delay time which depends on both the height and width of the sample. In this case 
the gel is supported by both the base and the side walls. We expect to find the longest delay times in this regime as the gel is being supported from the sides and the base simultaneously.

We may expect to find creeping behaviour within this regime when the height and width of the sample are much smaller than the crossover values. However, there is no way to predict where this may happen through this theory.

\section{Comparison With Experiment}

By solving the equations of motion for the continuum gel, Evans was able to give a theoretical estimate of the crossover height and width for the sample studied in this section $\left(\phi=0.20, C_{p}=7.14 \mathrm{mgcm}^{-3}\right)$. He found that for this sample $h_{x o} \approx 4 \mathrm{~mm}$ and thus $w_{x o} \approx 8 \mathrm{~mm}$. This compares well with the experimentally determined length-scales of $h_{x o} \approx 5 \mathrm{~mm}$ and $w_{x o} \approx 10 \mathrm{~mm}$.

As expected we found that the delay time was shortest in the size-independent regime ( $\bar{\tau}=185 \pm 23$ minutes). The delay time in the regions where extra support was supplied by either the walls or the base was significantly higher.

Below the crossover width we found width-dependent delay times. However, this was only observed when the initial sample height was below $20 \mathrm{~mm}$. Above, $h(0)=20$ $\mathrm{mm}$, in what should be the width-dependent-height-independent regime, the delay time appeared independent of width $(\bar{\tau}=406 \pm 91$ minutes $)$.

Two further disagreements with the expected behaviour were found in the thinnest samples. Firstly, as previously mentioned, the $5 \mathrm{~mm}$ wide sample consistently exhibited delay times much shorter than expected. This was attributed to the difficulty in adequately homogenising this sample. We also found that the $7 \mathrm{~mm}$ sample exhibited a height-dependent delay time in a region where we expected height-independence. This is shown in figure 6.7. We also found that this sample showed creeping behaviour at a height of $10 \mathrm{~mm}$. Although the theory makes no explicit predictions about where creeping behaviour should be observed we expect creeping to occur when $h(0) \ll Z_{\text {cross }}$.

Due to the small magnitude of the crossover length-scale we were unable to explore the regime in which both the sides and the base of the sample provide extra support. 


\section{Chapter 7}

\section{Discussion}

\subsection{Introduction}

This thesis is the result of a set of experimental observations which attempt to further our understanding of the collapse of transient gels. In carrying out these experiments we have uncovered new information on the way in which transient gels collapse but we are still little closer to formulating a quantitative model which could describe the fundamental processes involved.

Transient gelation and delayed sedimentation are phenomena which have been observed in many different weakly aggregated systems. Experimental studies on such systems have found many features similar to those observed by us. In this chapter we review the findings of different studies of gel collapse within the framework of our own observations. Based on what is currently known from these studies we then propose a qualitative picture to explain the occurrence of delayed sedimentation and creeping sedimentation in transient colloid-polymer gels.

Although our findings, and those of other studies, provide some insight into the collapse of transient gels, the overall picture is still incomplete. Thus much of what is contained within this chapter is speculative in nature. However, in making such speculation it is our intention to provide a foundation upon which further investigations of gel collapse may be built. 


\subsection{Initial Structure of Transient Gels}

In order to understand the evolution of gel structure during its lifetime it is useful to know its early-time structure. Here we present our model of the initial structure of a transient gel.

In the diffusion limited cluster aggregation model of gelation, clusters of particles can grow fractally until they reach a size where they touch and span the sample volume. At this point we think of the gel as being made of a close packed arrangement of clusters of size $R_{g e l}$, see figure 7.1. The size of the clusters $R_{g e l}$ therefore defines a maximum length-scale $l$ up to which the gel has a fractal structure. At length-scales above $R_{\text {gel }}$ the gel has a homogeneous structure. The weight of the gel is supported by a network of load bearing particle chains or "backbones". Each backbone of the structure is initially two or more particles diameters in width. Within each backbone the particles have a compact structure, see figure 7.1. This defines a minimum length-scale $b$, the width of the backbones, below which the structure is homogeneous. Thus the gel is fractal within the range of length-scales $b<l<R_{\text {gel }}$.

Not all particles in the gel are to be found in the load bearing chains of the structure. There are also non-load-bearing "tendrils" which are connected to the network but are non-percolating. Thus the tendrils contribute to the weight of the network but not to its structural rigidity.

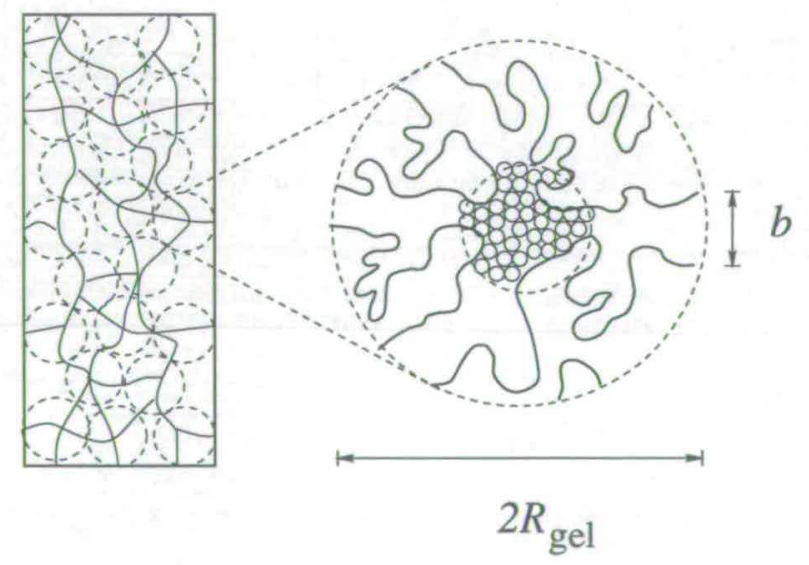

Figure 7.1: Schematic diagram showing the early-time structure of a transient gel. The gel is made from a randomly close packed arrangement of fractal clusters of size $R_{g e l}$ whose arms have a characteristic width $b$. Figure reproduced from [16]. 


\subsection{Delayed Sedimentation}

Previously it was believed that transient gels remained in a quiescent state throughout the delay period. However, our dark-field observations have shown that gels are far from dormant during this time. Regions of local breaking of the gel such as channels and streamers have been observed in other systems which show delayed sedimentation. In this section we present what is currently known about systems which undergo delayed sedimentation and propose some mechanisms which may explain the formation and growth of channels and streamers.

\subsubsection{Channels}

The presence of channels during settling is often indicated by a distinct maximum in the Tory plot [45]. This is a plot of interface settling velocity against the height of the settling interface. When channels break through the surface of the suspension they provide a quick route for the continuous phase to leave the suspension which increases the settling rate of the suspension.

Figure 7.2 show Tory plots for the samples discussed in chapter 4 , see table 4.1 for sample compositions. From the Tory plots we see that all samples which showed a delay time before rapid settling show a distinct maximum. These were also the samples in which channels were observed. The Tory plot of a sample which exhibited creeping sedimentation shows no such peak, solid line in figure 7.2. It is known from our observations that suspensions which show creeping behaviour are devoid of channels.

Holdich and Butt [46] have investigated the effects of channelling in suspensions of calcite. By comparing the settling behaviour of suspensions of a particular concentration with and without stirring, they found that the presence of channels increased the rate at which the suspension settled. Stirring the sample hindered the formation of channels. For the UN-stirred suspension, they found small increases in settling velocity with time indicating that channels may be present. They also found that the number of channels observed decreased as the concentration of the suspension increased.

Channels have been observed in other systems which display delayed sedimentation be- 


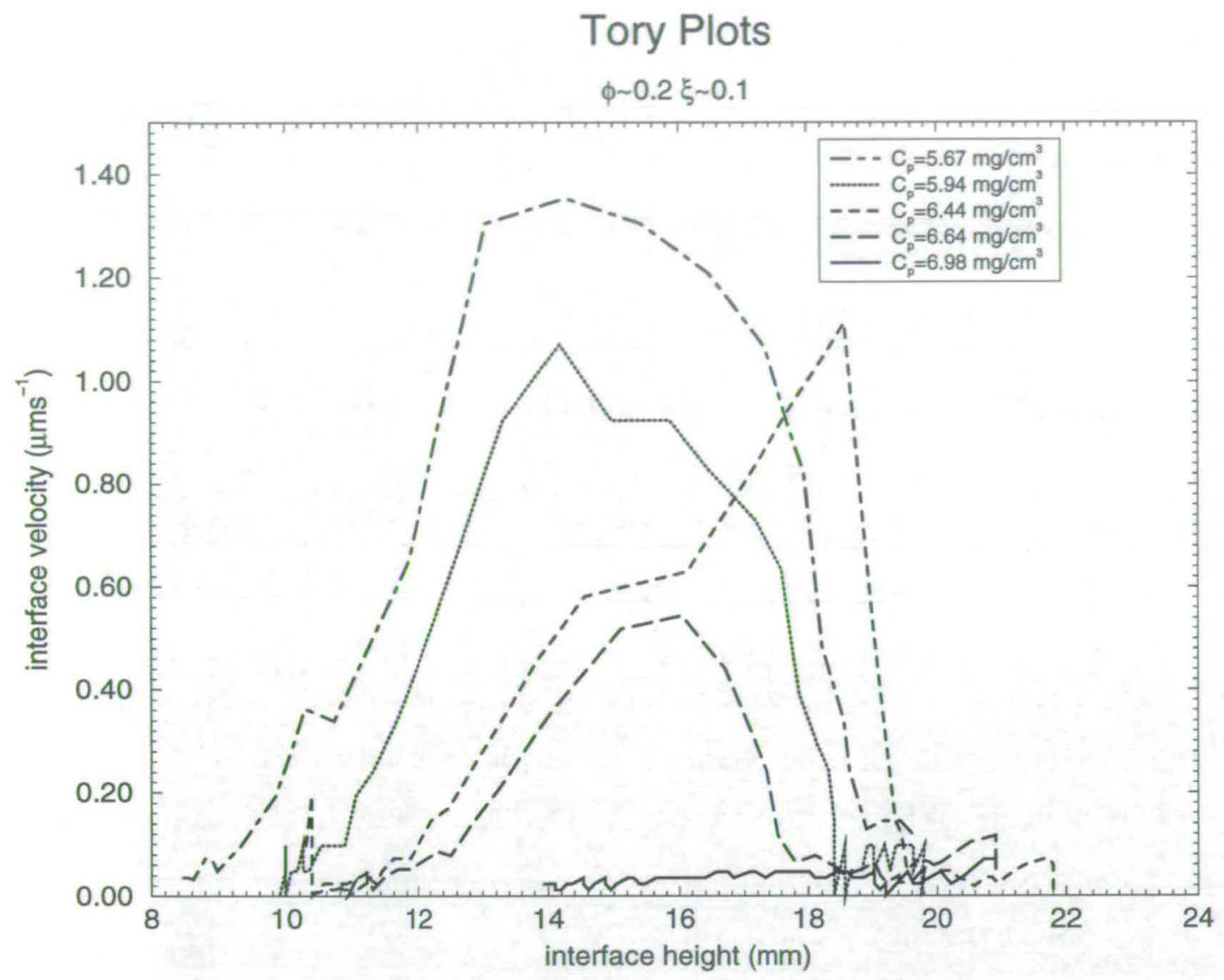

Figure 7.2: Plot of interface velocity against interface height. dot-dashed line: $C_{p}=5.67$ $\mathrm{mgcm}^{-3}$ ( $\left.\tau=200 \mathrm{~min}\right)$, dotted line: $C_{p}=5.94 \mathrm{mgcm}^{-3}(\tau=460 \mathrm{~min})$, short dashed line: $C_{p}=6.44 \mathrm{mgcm}^{-3}(\tau=1000 \mathrm{~min})$, long dashed line: $C_{p}=6.64 \mathrm{mgcm}^{-3}(\tau=1200 \mathrm{~min})$, solid line: $C_{p}=6.98 \mathrm{mgcm}^{-3}$ (creeping sedimentation).

haviour. Glasrud et al. [24] report the formation of channels in flocculated suspensions of iron oxide particles. They found that small bubbles of air entrained in the suspension by mixing formed vertical paths which then filled with oil. When the bubbles reach the particle-oil interface they produce small volcano-like features as shown by the image in figure 7.3 which shows a view from directly above of a suspension of iron oxide particles of volume fraction $\phi=6 \%$ (figure reproduced from [24]).

At lower particle concentration it was found that the width of the volcanoes was smaller but the number of volcanoes was significantly higher. The volcanoes on the top surface of the suspension also appeared closer together in the suspensions of lower concentration. 


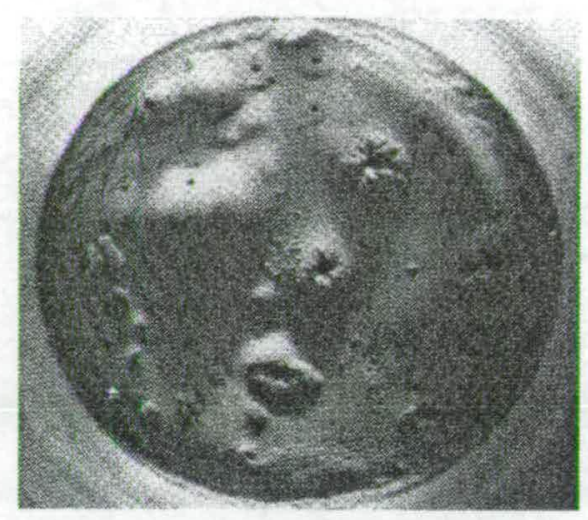

Figure 7.3: Image showing the volcanoes produced when channels break through the surface of a suspension of iron oxide particles. The diameter of the tube is $20 \mathrm{~mm}$. Figure reproduced from [24].

The formation of channel-like features has also been observed by Allain and co-workers [21] in aqueous suspensions of calcium carbonate which exhibit delayed sedimentation. They have found that the onset of rapid collapse corresponds to the formation of a fracture in the suspension [47]. The fracture then provides the suspending liquid with an easy route to the suspension surface.

By studying gelled samples in containers with large cross-sectional area, Cloitre [48] has found that these channel-like features have a characteristic spacing as if they were formed on a lattice. This characteristic spacing of channels may be explained by considering the variation of stresses in the gel structure in the vicinity of a channel.

Let us consider the stresses acting upon a small region of a gelled network when a channel forms nearby. The formation of a channel in the gel relaxes stresses on the structure at the point where it forms. However, away from the channel the stress on the structure increases as shown schematically by the solid line in figure 7.4(A). Once a channel forms, solvent from the neighbouring suspension will flow towards it and then escape to the surface. This flow creates mechanical stress on the network which decays with the distance from the channel as shown by the dashed line in figure 7.4(A). The combination of these two processes leads to a peak in the stress experienced by the gel network some characteristic distance $r^{\prime}$ from the site of the original channel as shown in figure 7.4 (B). The magnitude of $r^{\prime}$ will depend upon the mechanical properties of the gel and the size of the channel. 

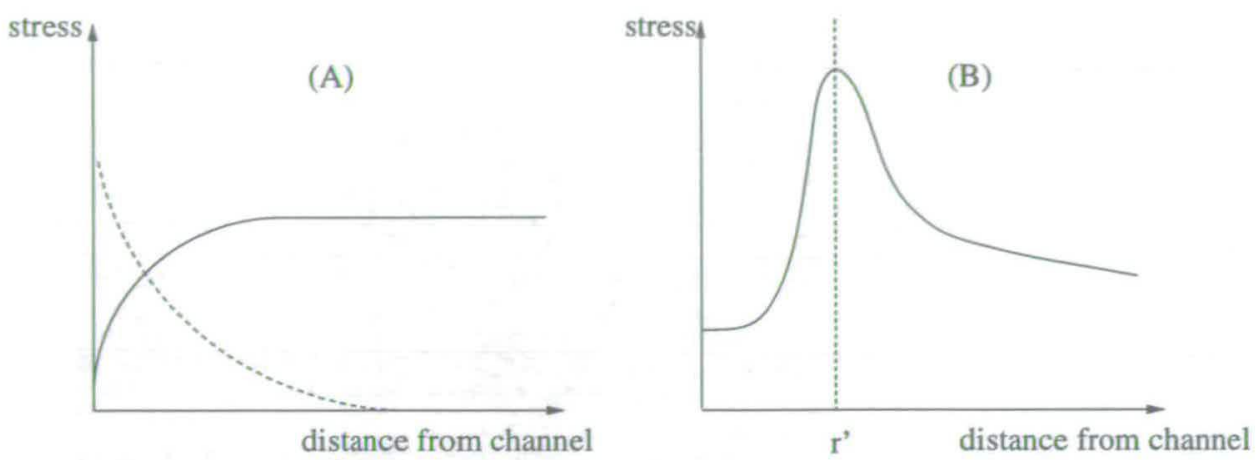

Figure 7.4: (A) The formation of a channel in the gel relaxes the stress on the structure where it forms. Further away from the channel the stress begins to build up again (solid line). Solvent then flows from the surrounding material into the channel increasing the stress in this region which then decays with distance from the channel (dashed line). (B) The combination of these two processes leads to a peak in the stress on the material a characteristic distance $r^{\prime}$ from the site of the original channel.

\section{Channel Formation}

In the suspensions of flocculated iron oxide particles studied by Glasrud et al. [24], air bubbles trapped in the suspension by mixing created paths which then filled with the suspending oil to form channels. Conversely Allain and co-workers [21] observe fracture of the gel which created channel-like features for water to escape from the suspension. Our observations suggest that channel formation in this colloid-polymer system progresses by a different mechanism. On one occasion we have observed the formation of a channel by a bubble of trapped air. However, this channel formed much faster than usual (seconds rather than tens of minutes) and was about twice as wide as those channels not formed by air bubbles. The channel was also very straight whereas most other channels show some deviation from the straight vertical path, see figure $4.6(\mathrm{e})$.

Channel formation in our system may be a result of flow-induced rearrangement of the particle network. As the gel slowly settles so the solvent must travel upwards in order to conserve mass. The driving force for this flow is provided by the pressure difference created by the weight of the particle network. The solvent will preferentially select the path of least resistance through the interconnected porous structure of the gel. On the length scale of the clusters, flow of solvent through the porous gel causes the pores to increase in size due to erosion of non-percolating material from the surface of the 
particle network. This will have the effect of decreasing the resistance to flow and may also lead to breaking of interparticle bonds. This local breaking of bonds may then be exploited by the solvent flow to form a channel.

\section{Channel Propagation}

The idea of erosion by solvent flow may also explain the shape of the channel height against time plots in figure 4.10 . We recall that channels propagate slowly at first. Around 50 minutes after formation the propagation velocity of the channel suddenly increases by a factor of 10 .

This yield-like behaviour may be explained as a consequence of "positive feedback" caused by erosion. Increasing the pore size by erosion will gradually increase the rate at which solvent can flow and thus the speed of the channel propagation. Eventually, the flow of solvent will be strong enough to break the interparticle bonds thus suddenly increasing the speed of channel propagation. In such a way positive feedback can lead to the yield-like behaviour observed in channels.

We showed that the curves of channel height against time could be scaled to fit a master curve, see figure 4.10. Thus the propagation of channels in the gel does not depend on where or when they form. This implies that the mechanical properties of the gel do not change significantly during the time where channels are forming. This suggests that the coarsening mechanisms involved in gel aging and collapse must be able to maintain the structural integrity of the particle network far into the delay period, see section 7.6.

\subsubsection{Streamers}

In our dark-field observations we have found that the onset of rapid collapse is triggered by the rapid sinking of streamer-like features into the suspension. These streamers cause macroscopic circulation and back-flow of the broken gel material. Similar circulation and back-flow of material has been observed by Glasrud et al. [24] during the rapid settling stage of collapse. Just before the onset of rapid collapse they observe local movement in the particle rich region which triggers global rearrangement and collapse. This local movement may be what we have termed streamers. 


\section{Streamer Formation}

Ultrasonic concentration profiling of gels performing delayed sedimentation has shown that regions of streamer formation can be up to $5 \%$ more concentrated than the surrounding gelled material. There are several mechanisms whereby dense material may be formed at the top of the suspension.

We know that channels cause local breaking of the gel structure when they break through the interface between the colloid-rich suspension and the supernatant. Fragments of broken gel have a more compact structure than the gel itself. Thus channels deposit dense material on the top of the suspension. This material will then sink and cause further local breaking which may lead to the formation of streamers.

Gel breaking at the top in samples with a meniscus may also be caused by a thin film of colloidal material which is present above the meniscus. Mixing of the sample always leaves a thin film of the suspension stuck to the sample cell walls. Once the sample is placed upright, this material slides down the walls and is deposited on top of the suspension. This may cause early time mixing of the upper region of the gel in samples which have a meniscus.

High magnification observations of the regions just underneath the sample meniscus have revealed small scale circulation of material. Material appeared to move along the base of the meniscus from its centre to the top corners before falling back into the suspension. This small scale circulation may also locally break the gel structure creating dense material at the top and may explain why streamer formation appears predominantly in the top corners of the suspension. This motion of colloids may be driven by a depletion force between the particles and the meniscus [49] since depletion between a curved interface and a colloidal sphere causes the particle to move towards higher curvature.

Some observations of full sample cells, i.e samples without a meniscus, have shown that streamers still form at the top. In this case we expect that channels would be the only mechanism for depositing dense material at the top of the suspension. We would therefore expect the initial formation of a layer of concentrated material to take longer than in samples with a meniscus. Also, the streamers in full samples may not 
necessarily propagate from the corners. More observations are required to confirm this speculation.

\section{Streamer Propagation}

Meeker [16] has speculated on the mechanics of streamer propagation. He has suggested that yield-like behaviour shown by streamers is a consequence of the shear thinning nature of colloid-polymer mixtures. This was touched on briefly in section 4.7.2 and is another example of positive feedback. Meeker considered the motion of a dense cluster sinking into a progressively shear-thinning material [16]. As the cluster sinks into a shear thinning suspension so it creates local flow which reduces the local viscosity of the suspension. Thus the cluster can sink at a faster rate further reducing the local viscosity of the suspension.

By numerically simulating the motion of a dense cluster through a shear thinning material, Meeker [16] showed that a sharp transition from a slow settling regime $\left(u_{s} \approx\right.$ $\left.0.02 \mu \mathrm{ms}^{-1}\right)$ to a fast one $\left(u_{f} \approx 0.8 \mu \mathrm{ms}^{-1}\right)$ was possible. His plot of cluster height with time shows qualitative agreement with our plot of streamer height with time.

\subsubsection{Cracking}

At high enough polymer concentration we have observed cracking of samples which undergo delayed sedimentation, see section 4.5.2. These cracks predominantly form at the inner cell wall and propagate into the bulk, see figure 4.7. This suggests that wall stresses play an important role in supporting the structure of long lived gels. The increasing importance of support from the walls and base of a settling network was key to our understanding of size-dependent delay times in chapter 6 . At early times these cracks form in the upper regions of the suspensions. However, as time passes the cracks begin forming lower down in the sample.

Glasrud et al. [24] have also observed horizontal cracks forming on the outer surface in their iron oxide suspensions. These cracks which are between $5 \mathrm{~mm}$ and $10 \mathrm{~mm}$ in length begin to form in the top half of the suspension at first. As time progresses cracks are seen forming in the lower regions of the sample. They also observe smaller cracks 
filled with oil forming between the channels.

The formation of macroscopic horizontally oriented fractures within transient colloidpolymer gels has been observed by Verhaegh et al. [50] using fluorescence confocal scanning laser microscopy (CSLM). They find that the number of fractures increases with time and they speculate that the fractures eventually weaken the gel to the extent that it collapses.

\subsubsection{Bright Bands}

The formation and growth of a layer of bright material (figure $4.6(\mathrm{~m})-(\mathrm{o})$ ) at the bottom of the sample during the delay time indicates compaction of the lower regions of the gel due to slow settling. This is confirmed by ultrasonic concentration measurements which show a slight rise in colloid concentration in the bottom millimetres during the delay time (figure 5.2). This feature is more pronounced for longer lived gels.

Robins et al. [22] have also observed the formation of a layer of concentrated "cream", i.e. oil droplets, forming at the top of oil-in-water emulsions displaying delayed creaming. However, during this time the bottom oil-water interface does not move. The formation of the cream layer in this case was attributed to a slight decrease in oil concentration in the upper half of the dispersion.

\subsection{Size Effects}

Size-dependent delay times similar to those reported in this work have also been observed in other weakly aggregated systems.

The dependency of delay time on the width of the sample has been observed by Glasrud et al. [24]. They compared the settling profiles of suspensions of flocculated magnetic iron oxide particles in cylindrical tubes of diameter $20 \mathrm{~mm}$ and $10 \mathrm{~mm}$. They found that at low particle concentration $(\phi=1 \%)$ there was a negligible effect. At higher concentration, the delay time observed before the onset of rapid settling increased when the suspension was placed in the narrower tube. This effect was more pronounced at higher concentration. 
Robins and co-workers [51] have found a dependency of delay time on sample width in polymer-flocculated oil-in-water emulsions.

\subsection{Mechanisms for Restructuring of Transient Gels}

Transient gels do not collapse immediately after formation which suggests that initially the structure is capable of supporting itself. However, some finite time later the gel is suddenly incapable of supporting itself and collapses to form a dense sediment.

In the $2 \mathrm{D}$ reversible-DLCA simulations of Haw et al. [19] the gelled structure once formed continued to break and reform due to thermally induced rearrangements until the end of the simulation. Without further coarsening of the network, the breaking and forming process would continue for all time and the gel may never collapse. We now consider some restructuring processes which may be significant in the aging and depercolation of transient gels.

\subsubsection{Thermally Activated Coarsening and Sintering}

This reversible-DLCA simulations of Haw et al. [19] provide some insight into the effects of thermal rearrangement on the structure of aggregated systems. In the absence of thermal rearrangements (irreversible-DLCA) tenuous fractal-like structures are formed where each arm of the structure is only one particle thick, see figure $2.7(\mathrm{~A})$. When the bond strength is of the order of a few $k_{B} T$ thermally activated rearrangement leads to the compaction of fractal-like structures (figure 2.7 (B)) since multiple particle bonds are more stable to thermal breakup than single bonds. This process can eventually lead to the depercolation of the space spanning cluster.

The time $\tau_{d p}$ over which thermally induced depercolation of the gel structure occurs is related to the probability of thermally activated bond breaking which follows a Boltzmann distribution $\sim \exp \left(-U / k_{B} T\right)$ where $U$ is the bond energy. Thus as the bond strength $U$ increases (by increasing the polymer concentration $C_{p}$ ) so the time it takes the gel structure to depercolate increases exponentially. In the absence of gravitationally induced restructuring, the depercolation time $\tau_{d p}$ may be identified as the delay 
time since the structure would be unable to support its weight in the presence of a gravitational field at this time.

We can gain further insight into thermally activated coarsening mechanisms by considering the processes involved in sintering [52]. This is a technique used to strengthen fractal-like materials such as silica aerogels by reducing the size of the pores in the structure. The work of Olivi-Tran et al. [53] considers the effect of various sintering mechanisms on materials initially made up from fractal arrangements of aggregated particles. Heating such porous materials at a temperature lower than the melting temperature gradually eliminates the pores and produces a net shrinkage of the structure. Gradual compactification of the porous structure formed by cluster aggregation may occur by a similar process.

On the scale of individual particles sintering may proceed via several mechanisms such as evaporation of particles from the structure and their subsequent deposition at other positions, surface diffusion of particles and viscous flow of material in the bulk of the percolated structure. Here we discuss the effect of the latter two processes on the structure of a fractal cluster.

\section{Surface Diffusion}

On the length-scale of the particles, surface diffusion of material may occur due to particle rolling driven by the surface tension of the cluster arms, see top right hand sketch in figure 7.5. Particles may also be able to make small thermally activated hops into spaces in the cluster arms as shown in the bottom right hand sketch in figure 7.5.

Olivi-Tran et al [53] investigated the effect of surface diffusion on 2D mass fractals. In their simulations the diffusive transport of material along the surface of the fractal objects was driven by pressure inhomogeneities associated with changes in curvature along the surface. The sintering process ended when a circular cluster had formed, i.e. a state of constant curvature was reached. 

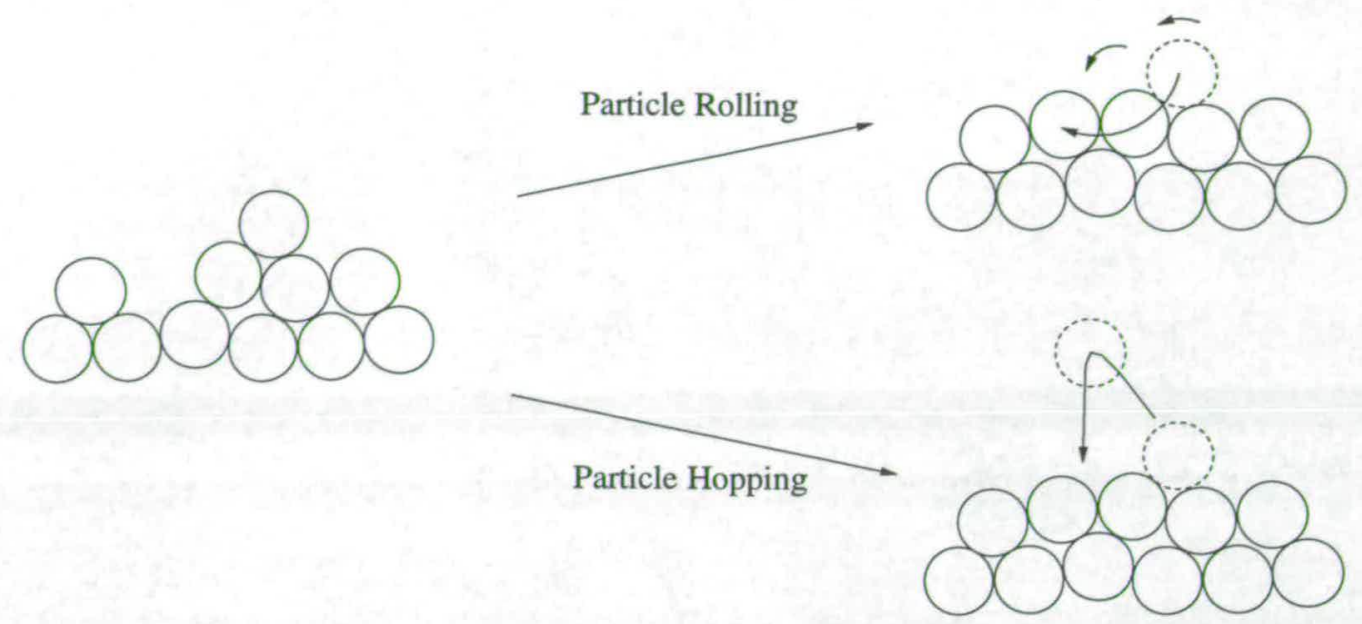

Figure 7.5: Diagram showing how surface diffusion of material along the arm of a cluster may occur via particle rolling (top) of particle hopping (bottom).

\section{Viscous Flow}

Sintering by viscous flow is also driven by surface tension and results in the formation of a spherical object. In this process, material is transported by viscous flow within the bulk of the cluster.

Figure 7.6 shows the evolution of a 2D mass fractal by surface diffusion (top) and viscous flow (bottom). From this figure it is clear that both mechanisms lead to a coarsening of the fractal backbone, i.e. the lower fractal cutoff length $b$ gradually increases. However, only surface diffusion can preserve the size of the fractal $2 R$ well into the sintering process. Viscous flow leads to significant shrinking of the cluster. Thus, in a system of percolating fractal objects, viscous flow would lead to break up of the structure after minimal rearrangement while surface diffusion processes would maintain the percolated structure for longer.

\section{Erosion}

A sintering-like coarsening process may also be driven by erosion of the fractal surfaces of the percolated structure due to the flow of solvent induced by the weight of the particle network. This idea has already been discussed as a mechanism for channel formation. At first the effect of erosion will be to incorporate the tendril material into 


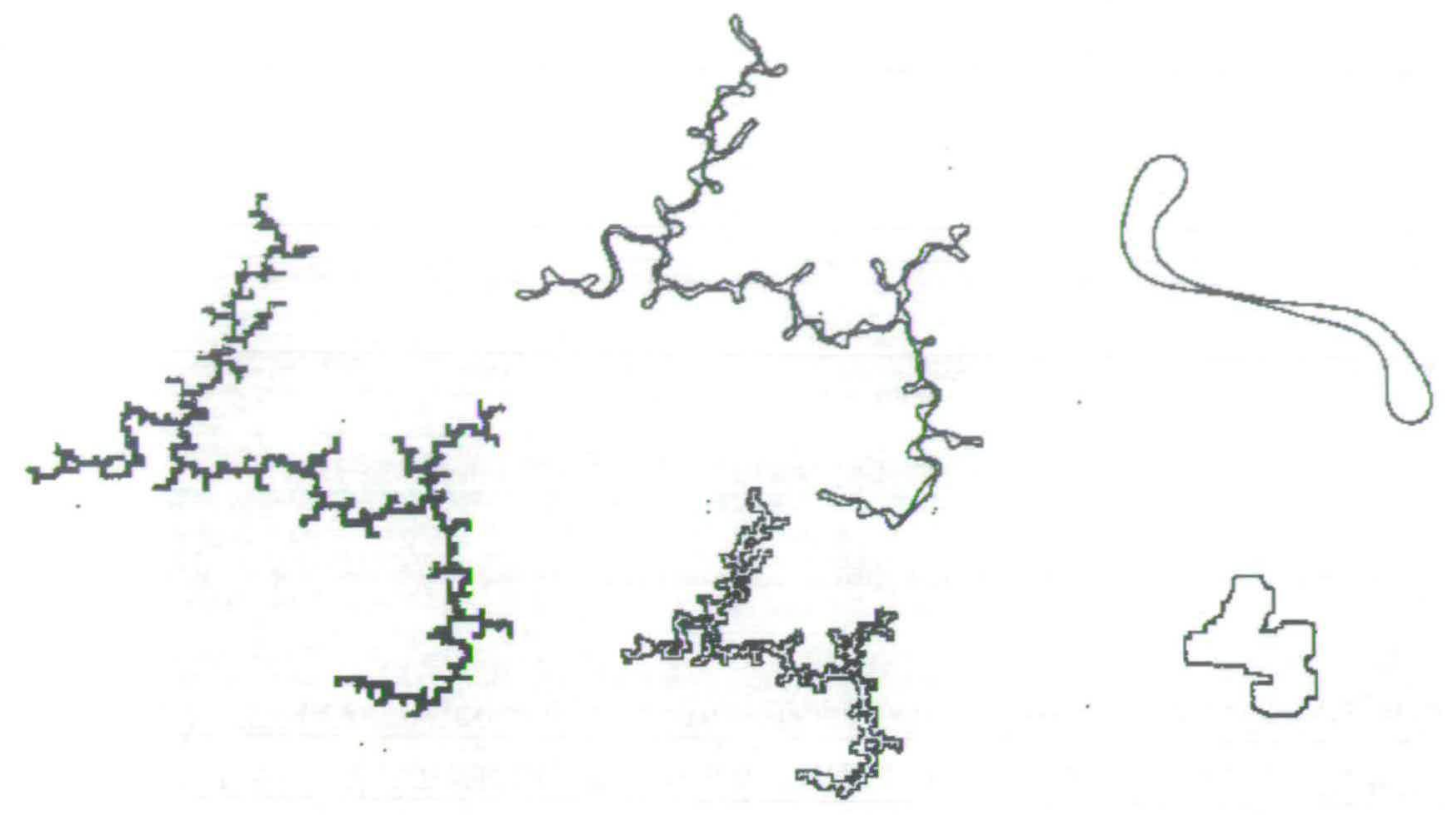

Figure 7.6: Evolution of a 2D mass fractal (left) by surface diffusion sintering (top) and viscous flow sintering (bottom). Both coarsening mechanisms increase the width of the fractal backbones of the gel. However, surface diffusion preserves the size of the fractal $2 R$ well into the sintering process while vicious flow causes the object to shrink continuously. Figure reproduced from [53].

the load-bearing backbones of the structure thus decreasing the resistance to flow and allowing solvent to flow at a faster rate through the structure. Further erosion may result in the breaking of the structure at weak (thinner) points which could eventually contribute to depercolation of the network.

\subsubsection{Fluid Instabilities}

Meeker [16] suggested that the retraction of the tendrils into the load-bearing structure may cause the gel to depercolate. He argued that backbones of the transient gel could be thought of as long thin cylinders of fluid. These cylinders would be unstable to small perturbations in their surface tension and thus break up and form individual drops. This is known as the Rayleigh instability. However, a steady flux of material from the non-percolating arms of the gel may be able to counteract this instability until all the non-percolated material is gone. The main backbones could then break into smaller clusters, i.e. the gel would depercolate. 


\subsubsection{Weight-Induced Coarsening}

Under the influence of gravity the weight of the particle network can also lead to coarsening.

By compressing thin tenuous particle chains with little rigidity it is possible to create thicker particle chains as shown in figure 7.7. In this way the structure gains more resistance to deformation and thus resists further settling. However, for small deformations the chains would behave elastically and may be prone to fracture if the compression force is high enough.

Further strengthening of the gel structure may occur due to compression. It is possible that non-percolating arms of the structure which contribute weight but no structural support may be converted into weight bearing backbones by compression, see figure 7.8. Flexible, tenuous backbones may also buckle under the compressional forces of settling and form loops of particle chains. These formation of loops would increase the structural rigidity of the gel.

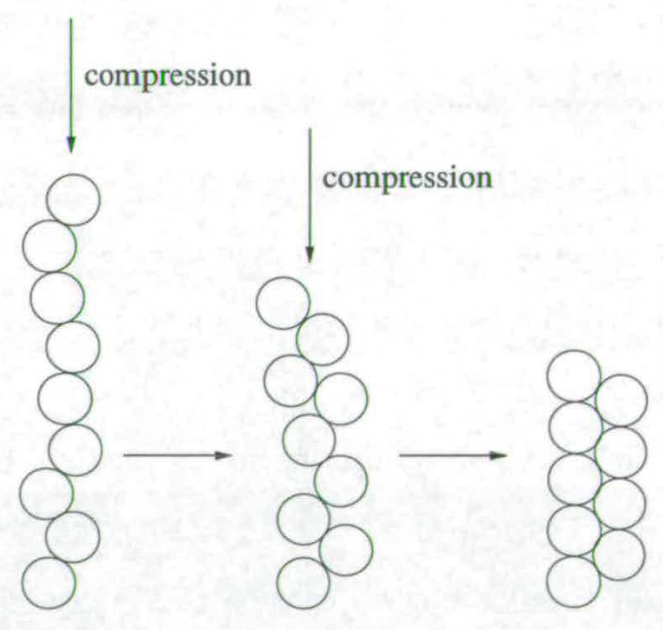

Figure 7.7: Schematic diagram showing how tenuous particle chains may form more rigid thicker chains under compressional forces. 


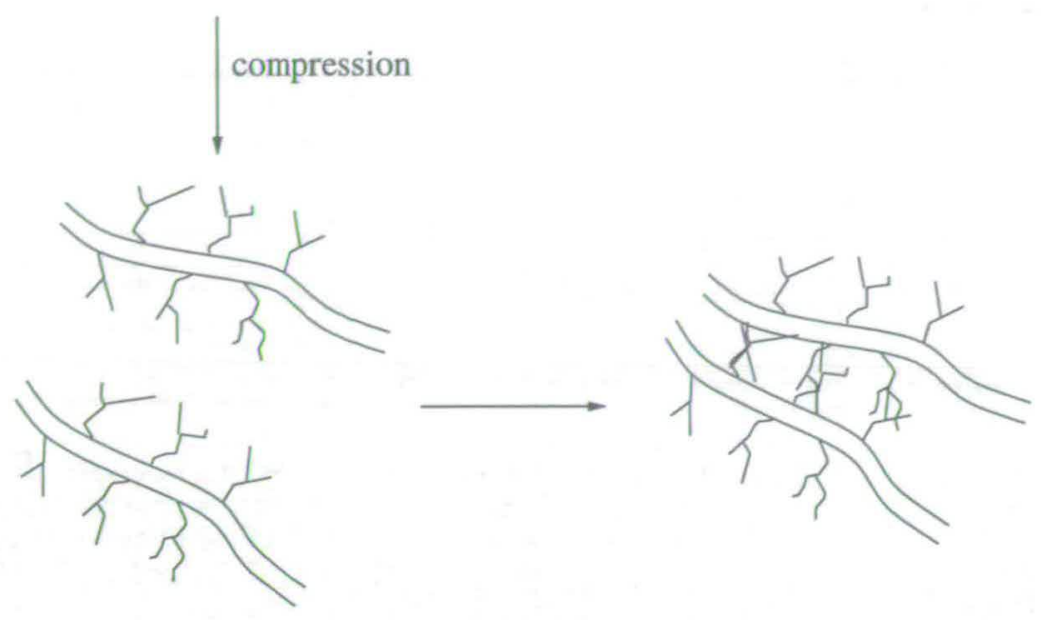

Figure 7.8: Schematic diagram showing how non-percolating arms of a fractal-like gel structure may be converted to percolating load-bearing arms by compression.

\subsection{A Qualitative Picture of Delayed Sedimentation in Transient Gels}

By appealing to some of the proposed coarsening mechanisms we now make some speculation on the origin of delayed sedimentation in transient gels

Initially the gel is composed of a close packed arrangement of fractal-like clusters of radius $R=R_{\text {gel }}$. Under gravity the gel is initially able to resist weight-induced settling via a percolated network of load-bearing particle chains of width $b$. There are many non-percolating tendrils attached to the particle network which add to the weight of the gel but do not provide any structural support. The characteristic pore size may be considered to be the size of the gaps between the particle branches of the network. Initially there will be a distribution of pore sizes with large pores created between the percolating backbones and smaller pores between the many tendrils.

Let us now consider what happens to the structural stability of the gel as it coarsens. The effect of coarsening by surface diffusion of material will be to withdraw the tendrils into the main structure. This will increase the fraction of material contributing to the structural support of the gel. The width of the load-bearing chains $b$ will increase as will the characteristic pore size as the non-percolating arms of the structure are eliminated. We therefore expect the gel to initially strengthen as suggested by Meeker's low shear viscosity measurements [16]. Slow compression due to settling of the gel will also strengthen the network. 
At this time the gel is still able to support itself by the network of particle chains. Small local breaking of the structure due to channels and the beginning of streamers can be accommodated and we think of the gel as being able to "heal" itself. Thermally activated breaking of the gel in two or more large clusters as observed by Haw [19] can still be counteracted by recombination of the clusters.

The increase in the characteristic pore size reduces the resistance to flow of solvent thus gradually increasing the importance of erosion. This along with further coarsening due to surface diffusion eventually leads to a case where all the non-percolating material has been incorporated into the load-bearing structure. From now on further coarsening leads to a shrinking of the percolating structures. The backbones begin to break and the structure weakens. The probability of the cluster being able to recombine is now very small and the ability of the gel to heal is greatly reduced. Local concentration instabilities such as streamers will begin to move at faster velocities.

Eventually the structure will depercolate and lose all its self supporting properties. The streamers suddenly can no longer be supported and they sink rapidly into the suspension triggering the onset of rapid collapse.

\subsection{Crossover from Delayed to Creeping Sedimentation}

In our model of delayed sedimentation we proposed that rapid collapse occurs once the gel structure is no longer strong enough to support concentration instabilities caused by local breaking, i.e. streamers. However, at high enough bond strength, or small enough sample size, the structure does not appear to lose its integrity in such a dramatic way and we observe creeping sedimentation behaviour. We propose that this change in settling behaviour can be explained by a change in the dominant coarsening mechanism.

As the amount of support the gel can gain from its own structure increases so the time-scale $\tau_{d p}$ for thermally activated coarsening increases. The amount of structural support available to the gel will depend on its size (height and width) and the interparticle bond strength $U$. Thus we expect the rate of thermally activated coarsening to be slower for stronger gels. During this time $\tau_{d p}$ the gel is also being strengthened by weight-induced rearrangements as discussed in section 7.5 .3 . 
Delayed sedimentation occurs when thermally activated weakening occurs faster than weight-induced strengthening. However, if thermal rearrangements become slow enough, the aging of the gel may be dominated by gravitational coarsening. In this case we expect the structure to gradually compress under its own weight.

\subsection{Suggestions for Future Work}

So far we have only considered transient gelation in colloid-polymer systems with short ranged interparticle interactions $(\xi \ll 0.1)$. The formation and collapse of gels with long ranged interactions may be different from the short ranged gels of which much is now known. A comparison of gelation in different $\xi$ systems may also provide a link between the work of this group and that of Lekkerkerker [54, 50] who have studied gelation in colloid-polymer mixtures with silica particles at large size ratios.

Extending our study to include gelation in polydispersed systems would also be useful. Some preliminary work on this has already been carried out by Fairhurst [7]. We have performed some preliminary observations on some colloid-polymer gels with low particle size polydispersity $(\sigma=10 \%)$ and have found similar features in delayed sedimentation to those found in monodispersed gels, i.e. channels and streamers. A more complete study of polydispersity could provide a link between our model systems and the very polydispersed emulsions of Robins and co-workers.

In trying to understand the yield-like settling behaviour of transient gels many rheological experiments have been performed $[55,56]$. However, the rheological studies have concentrated on shearing the samples and have provided little insight into the origin of the delay time. Perhaps a more useful experiment would be to study the rheology of a gel under compressional stresses.

Finally, it would be useful to study gels under different gravitational conditions, i.e. vary the effect of gravity. This can be achieved in the laboratory by matching the density of the suspending fluid to that of the colloidal particles. Such a study would provide useful insight into the relative importance of thermally activated and weightinduced coarsening in the collapse of transient gels. 


\section{Chapter 8}

\section{Conclusion}

As has been previously noted this thesis is a collection of observations which were motivated by the need for more information on the ways in which transient gels collapse. Although we have been unable to answer many of the questions we set out to answer it is the hope of the author that this work may act as a foundation for future studies of gel collapse.

We have found two different types of settling behaviour. At low and intermediate polymer concentration above the gel boundary we found gels which undergo delayed sedimentation. At high enough polymer concentration the settling behaviour changes and rapid collapse is no longer observed. This behaviour was termed creeping sedimentation.

Using dark-field observation and ultrasonic concentration profiling we have studied the settling of both types of behaviour in an attempt to further understand the physics of transient gel collapse. We have found that dark-field imaging is a very useful technique with which to study the bulk behaviour of settling systems. This technique complements other more indirect measurements, such as concentration profiling, very well and we have found that our video images have given useful insight into the mechanisms of gel collapse.

In conclusion we briefly summarise our main findings. 


\section{Delayed Sedimentation}

Delayed sedimentation has been found to be a general behaviour observed in collapsing gels over a wide range of delay times (from 1 hour to 1 day). During the delay time the concentration remains constant and homogeneous throughout almost the whole height of the sample. This suggests that whatever aging processes may be responsible for the final collapse of the gel must occur homogeneously throughout the particle network.

Channels provide routes by which solvent can easily escape the suspension. The plot of channel height with time shows yield-like behaviour. This may be a consequence of increasing pore size due to solvent driving non-percolating arms of the gel into the load-bearing structure by erosion. We have also found that the channel height against time plots of different channels scale onto one master curve suggesting that the mechanical properties of the gel do not change significantly throughout the period of channel formation.

The formation of streamers is the mechanism by which the gel eventually collapses. Regions of streamer formation have been shown to correspond to areas of slightly higher than average concentration. Why these concentration fluctuations should form at the top is not clear although we have proposed a few possible mechanisms.

As an explanation of the yield-like settling behaviour we have speculated that thermally induced coarsening first strengthens and then eventually weakens the particle network to such a degree that weight-induced fluctuations such as streamers can no longer be supported. The streamers then rapidly sink into the gel causing global mixing and triggering the onset of rapid collapse.

The early stages of rapid collapse are characterised by circulation and back-flow of material which gradually slows down as the suspension settles and a dense sediment is deposited at the bottom of the sample. Ultrasonic measurements have shown the persistence of a layer of constant concentration which after time develops a gradient in concentration. We proposed that the sample behaves as a suspension of polydispersed clusters which settle at different velocities once large scale circulation slows thus creating a gradient of concentration. 
Long lived gels show the same general behaviour as short delay time gels but over longer time-scales. We also observe the formation of cracks at the inner wall of the sample cell suggesting that stronger gels may be able to gain more support against settling from wall friction.

\section{Creeping Sedimentation}

Creeping sedimentation has been observed at high polymer concentration and in samples which are very short or very narrow. The range of polymer concentration, height, or width over which the change from delayed sedimentation to creeping sedimentation occurs is observed to be small. We also found that samples near the boundary between the two settling regimes showed erratic behaviour.

Concentration measurements show that creeping sedimentation samples compress more or less uniformly. The lower region of the suspension is gradually crushed by the settling suspension above and thus a dense sediment is formed.

Observations show cracks forming at the inner walls and propagating into the suspension. The height of crack formation decreases as the suspension settles. A coarse, crumbled texture which corresponds to a region of raised concentration forms at the top and slowly sinks into the suspension mixing up the features that were previously present in the sample.

The reasons for this change in settling behaviour are unclear. We have speculated that the lack of yield-like behaviour may be due to a changeover from thermally activated coarsening, that leads to delayed sedimentation, to weight-induced restructuring.

\section{Size Effects}

An investigation into the height and width dependency of gel lifetime has shown that a particular gel can show both "weak" and "strong" behaviour depending on its dimensions with respect to a crossover length-scale $Z_{\text {cross }}$. For a gel of fixed width, there exists a crossover height $h_{x o}=Z_{\text {cross }}$ below which the properties of the gel, includ- 
ing $\tau$ will be dependent on height. Similarly, for a gel of fixed height there exists a crossover width $w_{x o} \approx 2 \times Z_{\text {cross }}$ below which $\tau$ will depend on initial sample width. We have found that the delay time increases with decreasing height and width in the size dependent regimes.

The existence of the crossover length-scale is explained by a recent theory. Weightinduced settling of the gel can be opposed by drag forces due to the flow of solvent from the gel and by the rigidity of the particle network. While the drag force will always be present, structural support will only have an effect if the particle network is capable of transferring stresses from the walls and base to the bulk of the sample. In the absence of structural support the gel will have size-independent properties. However, if the gel can support itself then the gel behaviour will be size-dependent. It is possible to define a length-scale $Z_{\text {cross }}$ below which the gel will show size-dependent properties. This length-scale depends upon the strength, lifetime and sedimentation rate of the network of particle chains.

For a gel with composition $\phi=0.2$ and $C_{p}=7.14 \mathrm{mgcm}^{-3}$ we find a crossover height of $h_{x o} \approx 5 \mathrm{~mm}$ and a crossover width of $w_{x o} \approx 10 \mathrm{~mm}$. These findings compare well with the theoretical predication for the crossover length-scale for this sample which is $Z_{\text {cross }} \approx 4 \mathrm{~mm}$, i.e. $h_{x o} \approx 4 \mathrm{~mm}$ and $w_{x o} \approx 8 \mathrm{~mm}$. 


\section{List of Figures}

1.1 Phase behaviour of a suspension of monodispersed hard-spheres. . . . . 3

1.2 A 2 dimensional representation of entropically driven freezing . . . . 4

2.1 The origin of the depletion attraction in colloid-polymer mixtures . . . 10

2.2 The common tangent construction and the lever rule . . . . . . . 13

2.3 Theoretical colloid-polymer phase diagram . . . . . . . . . . . . 14

2.4 Experimental phase diagram $\left(C_{p}\right.$ against $\left.\phi\right)$ for a colloid-polymer mixture 15

2.5 Phase diagram of a colloid-polymer mixture expanded to include nonequilibrium states. . . . . . . . . . . . . . 16

2.6 Schematic diagram showing the growth of a fractal cluster . . . . . 18

2.7 2D Reversible DLCA simulation of particle aggregation $\ldots \ldots \ldots \ldots$

2.8 Diagram of the free energy density against $\phi$ for large $C_{p}^{f r e e} \ldots \ldots 22$

2.9 Schematic sedimentation profile of a suspension exhibiting delayed sedi-

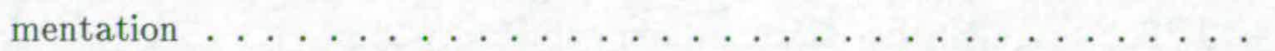

2.10 Delayed sedimentation: sedimentation plot and SALS . . . . . . . 24

3.1 The principle of a dark-field imaging technique ... . . . . 30

3.2 The depth of field of a single lens system . . . . . . . . . 31 
3.3 Measurement of the depth of field for a typical objective lens . . . . . 32

3.4 Dark-field viewing experimental setup . . . . . . . . . . . . . 34

3.5 Schematic diagram of the scattering geometry in the DOR . . . . . 36

3.6 Schematic diagram showing the set up of the Ultrasonic rig at IFR . . . 37

3.7 Speed of sound against temperature for distilled water at 1 atmosphere $\quad 38$

4.1 Colloid $\phi$ calibration by crystallisation method . . . . . . . . 48

4.2 Measuring the sedimentation profile . . . . . . . . . . 52

4.3 Extracting $\tau, u_{s}$, and $u_{f}$ from a sedimentation profile $\ldots \ldots \ldots$

4.4 Sedimentation profiles for a sequence of gels with volume fraction $\phi=0.256$

4.5 Plot of delay time against free volume polymer concentration . . . . . 57

4.6 Dark-field images of a gel undergoing delayed sedimentation . . . . . . 58

4.7 Dark-field images of a long lifetime gel . . . . . . . . . . . 60

4.8 Dark-field images of a gel exhibiting creeping sedimentation behaviour . 62

4.9 Graph of channel height against time for four separate channels . . . . . 64

4.10 Graph of shifted channel height against time $\ldots \ldots \ldots$. . . . . 65

4.11 Graph of streamer height against time for a gel with $\tau \approx 420$ minutes $\quad$. 66

4.12 Graph of bright band thickness against time . . . . . . . . . . 67

4.13 High magnification image of a bright band $\ldots \ldots \ldots 6$

5.1 Concentration profile of a gel with a delay time of $\tau \approx 3$ hours . . . . . $\quad 72$

5.2 Concentration profile of a gel with a delay time of $\tau \approx 8$ hours $\ldots . . . \quad 73$

5.3 Concentration profiles of a long delay time gel, $\tau \approx 25$ hours $\ldots . . .75$ 
5.4 Concentration profiles of a gel exhibiting creeping sedimentation $\ldots 77$

5.5 Contours of constant concentration $\tau=25$ hours . . . . . . . . 80

5.6 Contours of constant concentration of creeping sedimentation . . . . . 82

$6.1 \tau$ vs initial sample height $\ldots \ldots \ldots \ldots \ldots \ldots \ldots \ldots$

$6.2 u_{f}$ against initial sample height $\ldots \ldots \ldots \ldots \ldots \ldots$

6.3 Creeping sedimentation behaviour of short samples . . . . . . 86

6.4 Erratic settling behaviour $\ldots \ldots \ldots \ldots \ldots \ldots \ldots \ldots$

6.5 The crossover width $\ldots \ldots \ldots \ldots \ldots \ldots \ldots \ldots \ldots$

6.6 The crossover height $\ldots \ldots \ldots \ldots \ldots \ldots$

$6.7 \tau$ against initial sample height below the crossover width . . . . . . . . 91

6.8 The balance of forces on a settling slice of gel $\ldots \ldots \ldots \ldots$. . . . . 92

6.9 Gradient of settling velocity with height $\ldots \ldots \ldots$. . . . . . 93

6.10 The response of a gel to varying height and width $\ldots \ldots \ldots 5$

7.1 The early time structure of a transient gel . . . . . . . . . 98

7.2 Tory plots for a range of gelled samples . . . . . . . 100

7.3 Image of "volcanoes" on the suspension surface, from [24] . . . . . . . 101

7.4 The characteristic spacing of channels . . . . . . . . . 102

7.5 Mechanisms of sintering-like coarsening of gels . . . . . . . 109

7.6 Sintering if a $2 \mathrm{D}$ mass fractal, from $[53] \ldots \ldots \ldots \ldots \ldots$

7.7 Weight-induced coarsening of tenuous particle chains . . . . . . . 111

7.8 Weight-induced strengthening of the gel structure . . . . . . . 112 


\section{List of Tables}

3.1 Table of ultrasound velocity with different colloid volume fractions. The velocity of ultrasound is also shown for pure solvent and the polymer stock solution. ....................... 43

4.1 Table showing the volume fraction $\phi$, polymer concentration $C_{p}$ in $\mathrm{mgcm}^{-3}$, free volume polymer concentration $C_{p}^{f r e e}$ in $\mathrm{mgcm}^{-3}$, the typical delay time $\tau$ and the slow and fast settling rates, $u_{s}$ and $u_{f}$, of the samples to be discussed in chapter $4 \ldots \ldots \ldots \ldots \ldots 5$

5.1 Table of compositions and delay times for the samples studied by ultrasonic concentration profiling . . . . . . . . . . . . 71

6.1 Average delay time $\bar{\tau}$ in minutes as a function of initial sample height $h(0)$ and width $w(0) \ldots \ldots \ldots \ldots \ldots \ldots$ 


\section{Bibliography}

[1] M. S. Elliot. The Optical Microscopy of Colloidal Suspensions. PhD thesis, University of Edinburgh, 1999.

[2] W.C.K. Poon and P.N. Pusey. Phase transitions of spherical colloids. In M.Baus, editor, Observation and Simulation of Phase Transitions in Complex Fluids, pages 3-51. Kluwer Academic Publishers, 1995.

[3] W. W. Wood and J. D. Jacobson. Preliminary results from a recalculation of the Monte Carlo equation of state of hard spheres. Journal of Chemical Physics, 27:1207, 1957.

[4] W.G. Hoover and F. H. Ree. Melting transition and communal entropy for hard spheres. Journal of Chemical Physics, 49(8):3609-3617, 1968.

[5] J. Israelachvili. Intermolecular and Surface Forces. Academic Press, 2nd edition, 1991.

[6] P. N. Pusey and W. van Megan. Phase behaviour of concentrated suspensions of nearly hard colloidal spheres. Nature, 320:340-342, March 1986.

[7] D. J. Fairhurst. Polydispersity in Colloidal Phase Transitions. PhD thesis, University of Edinburgh, 1999.

[8] S. Asakura and F. Oosawa. Journal of Polymer Science, 33:183, 1958.

[9] A. Vrij. Polymer at interfaces and the interactions in colloidal dispersions. Pure and Applied Chemistry, 48:471-483, 1976.

[10] H. Reiss, H. L. Frisch, and J. L. Lebowitz. Journal of Chemical Physics, 31:369, 1959. 
[11] J. L. Lebowitz, H. Helfand, and E. Praestgaard. Journal of Chemical Physics, 43:774, 1965.

[12] H.N.W. Lekkerkerker, W.C.K. Poon, P.N. Pusey, A. Stroobants, and P.B. Warren. Phase behaviour of colloid + polymer mixtures. Europhysics Letters, 20(6):559$564,1992$.

[13] H.B. Callen. Thermodynamics and Introduction to Thermostatistics, chapter 9. John Wiley, 1985.

[14] W.C.K. Poon, J.S. Selfe, M.B Robertson, S.M. Ilett, A.D. Pirie, and P.N. Pusey. An experimental study of a model colloid-polymer mixture. Journal de Physique, 3:1075-1086, July 1993.

[15] W.C.K. Poon, A.D. Pirie, and P.N. Pusey. Gelation in colloid-polymer mixtures. Faraday Discussions, 101:65-76, 1995.

[16] S.P. Meeker. Low-Shear Rheology and Delayed Sedimentation of Colloidal Systems. $\mathrm{PhD}$ thesis, The University of Edinburgh, 1997.

[17] T. Vicsek. Fractal Growth Phenomena. World Scientific, 2nd edition, 1994.

[18] M. D. Haw. Computer Simulation of Aggregation and Gelation in Colloidal Suspensions. PhD thesis, University of Edinburgh, 1996.

[19] M.D. Haw, M. Sievwright, W.C.K. Poon, and P.N.Pusey. Cluster-cluster gelation with finite bond energy. Advances in Colloid and Interface Science, 62:1-16, 1995.

[20] W. C. K. Poon and M. D. Haw. Mesoscopic structure formation in colloidal aggregation and gelation. Advances in Colloid and Interface Science, 73:71-126, 1997.

[21] C. Allain, M. Cloitre, and M. Wafra. Aggregation and sedimentation in colloidal suspensions. Physical Review Letters, 74(8):1478-1481, February 1995.

[22] M.M. Robins, Kim Ng, P.A. Gunning, and A. Parker. Delayed creaming of oilwater emulsions containing polysaccharide. In Proceedings of A.C.S. SymposiumAdvances in Polysaccharides Characterization and Applications, 1995. 
[23] C. Allain, M.Cloitre, and M. Wafra. Sedimentation of colloidal suspensions in the presence of reversible aggregation. In H. Toulhoat and J. Lecourtier, editors, Physical Chemistry of Colloids and Interfaces in Oil Production, pages 259-261. Editions Technip, 1992.

[24] G.G. Glasrud, R.C. Navarrete, L.E. Scriven, and C.W. Macosko. Settling behaviour of iron oxide suspensions. American Institute of Chemical Engineers Journal, 39(4):560-568, April 1993.

[25] S. P. Meeker. private communication. Width dependent delay times have been found in colloid-polymer gels. These experiments were performed in rectangular cells where only one width dimension was changed.

[26] S. G. Lipson, H. Lipson, and D.S. Tannhauser. Optical Physics, chapter 12, pages 345-346. Cambridge University Press, 3rd edition, 1995.

[27] M. Pluta. Advanced Light Microscopy, volume 1, chapter 3, page 294. Elsevier Science Publishing Co., 1988.

[28] Image-grabbing software written by Dr. Mark Haw.

[29] A particularly cool name first used by Prof. Mike Cates.

[30] S. R. Reddy and H. S. Fogler. Emulsion stability - Experimental studies on simultaneous flocculation and creaming. Journal of Colloid and Interface Science, $82: 128,1981$.

[31] A.M. Howe and M.M. Robins. Determination of gravitational separation in dispersions from concentration profiles. Colloids and Surfaces, 43:83-94, 1990.

[32] G. W. C. Kaye and T. H. Laby, editors. Tables of Physical and Chemical Constants. Longmans, 14th edition, 1996.

[33] J. Blitz. Fundamentals of Ultrasonics. Butterworth and Co., 1963.

[34] D. J. Hibberd. Use of Ultrasound to Characterise Polymer Induced Flocculation. $\mathrm{PhD}$ thesis, University of East Anglia, 1997.

[35] R. J. Urick. A sound velocity method for determining the compressibility of finely divided substances. Journal of Applied Physics, 18:983, 1947. 
[36] D. J. McClements and M. J. W. Povey. Scattering of ultrasound by emulsions. Journal of Physics D, 22:38, 1989.

[37] D. J. Hibberd. private communication.

[38] G. C. Berry. Thermodynamic and conformational properties of polystyrene I: Light scattering studies on dilute solutions of linear polystyrenes. Journal of Chemical Physics, 44(12):4550-4564, 1966.

[39] W. Schaertl and H. Sillescu. Brownian dynamics of polydisperse colloidal hard spheres: Equlibrium structures and random close packings. Journal of Statistical Physics, 77:1007-1025, 1994.

[40] D. R. Lide, editor. CRC Handbook of Chemistry and Physics. CRC, 76th edition, 1995-1996.

[41] V. C. Martelozzo. private communication.

[42] A. A. Potanin, R. de Rooij, D. Van de Ende, and J. Mellema. Microrheological modelling of weakly aggregated dispersions. Journal of Chemical Physics, 102:5845-5853, April 1995.

[43] A. A. Potanin and W. B. Russel. Fractal model of consolidation of weakly aggregated colloidal dispersions. Physical Review E, 53(4):3702-3709, April 1996.

[44] R.M.L. Evans. private communication.

[45] E. M. Tory. Batch and Continuous Thickening of Slurries. PhD thesis, Purdue University, 1961.

[46] R.G. Holdich and G. Butt. An experimental study of channelling and solid concentration during the batch sedimentation of calcite suspensions. Transactions of the Institution of Chemical Engineers, 73(Part A):833-841, sept. 1995.

[47] M. Wafra. PhD thesis, Université Paris XIII, 1994.

[48] M. Cloitre. private communication to W. C. K. Poon.

[49] A. D. Dinsmore, A. G. Yodh, and D. J. Pine. Entropic control of particle motion using passive surface microstructures. Nature, 383:239-242, Sept. 1996. 
[50] N. A. M Verhaegh, D. Asnaghi, and H. Lekkerkerker. Transient gels in colloidpolymer mixtures studied with fluorescence confocal laser microscopy. Physica A, 264:64-74, 1999.

[51] P. Manoj. private communication.

[52] R. W. Cahn, P. Hansen, and E. S. Kramer, editors. Processing of Ceramics Part I, volume 17A of Materials Science and Technology: A Comprehensive Treatment. Weinheim, 1996. Volume Ed. R. J. Brook.

[53] N. Olivi-Tran, R. Thouy, and R. Jullien. Sintering of mass fractals by surface diffusion or by viscous flow: Numerical and scaling approaches in $\mathrm{d}=2$. Journal de Physique II, 6:557-574, 1996.

[54] N. A. M. Verhaegh, D. Asnaghi, H. N. M. Lekkerkerker, M. Giglio, and L. Cipelletti. Transient gelation by spinodal decomposition in colloid-polymer mixtures. Physica A, 242:104-118, 1997.

[55] W. C. K. Poon, L. Starrs, S. P. Meeker, A. Moussaïd, R. M. L. Evans, P. N. Pusey, and M. M. Robins. Delayed sedimentation of transient gels in colloidpolymer mixtures: dark-field observations, rheology and dynamic light scattering. Faraday Discussion, 112:143-154, 1999.

[56] P. Manoj, A. D. Watson, D. J. Hibberd, A. J. Fillery-Travis, and M. M. Robins. Characterization of a depletion flocculated polydisperse emulsion II: steady-state rheological investigations. Journal of Colloid and Interface Science, 207:294-302, 1998. 